\title{
Coerciveness inequalities for nonelliptic systems of partial differential equations.
}

\author{
John R. Schulenberger and OAlvin H. Wilcox (*) (**)
}

Ustract. - This paper deals with first-order matrix partial differential operators of the form

$$
L=-i \sum_{j=1}^{n} L_{j}(x) D_{j}+L_{\mathrm{o}}(x)
$$

where $x=\left(x_{1}, \ldots, x_{n}\right) \in R^{n}, D_{j}=\partial / \partial x_{j}$, and the $L_{j}(x), j=0,1,2, \ldots, n$, are $m^{\prime} \times m$ matrix-valued functions of $x$. Let $L_{2}, n_{3}$ be the Hilbert space of square integrable $m$-vector-valued functions on $R^{n}$. The operator (1) determines a closed linear operator $L: L_{2}, m \rightarrow L_{2}, n^{\prime} \cdot L$ is said to be coercive on a subspace $V \subset L_{2}, m$ if there is a constant $\mu>0$ such that

$$
\sum_{j=1}^{n}\left\|D_{j} u\right\|^{2} \leq \mu^{2}\left(\|L u\|^{2}+\|u\|^{2}\right)
$$

for all $u \in D(L) \cap V$, where $D(L)$ denotes the domain of $L$ and $\|\cdot\|$ denotes the norm in $L_{2}, m . L$ is said to have constant deficit $k$ in $R^{\text {nd }}$ if the symbol $L(p, x)=\underset{j=1}{\mathrm{\Sigma}} L_{j}(x) p_{j}$ has constant rank $m-k$ for all $x \in R^{n}$ and $p \in R^{n}-\{0\}(L$ is elliptic if and only if $k=0$ ). The paper gives criteria for nonelliptic operators $L$ of constant deficit $k$ to be coercive on subspaces. In particulav, operators of the form $A=-i E(x)^{-1} \sum_{j=1}^{n} A_{j} D_{j}$ are considered where $E(x)$ and $A_{j}$ are $m \times m$ Hermitian matrices and $E(x)$ is positive defi. nite. $A$ defines a self-adjoint operator on the Hilbert space $H$ with inner product $(u, v)_{E}=\int u^{*} E v d x$. It is shown, under switable hypotheses on $E(x)$ and $A_{j}$, that $A$ is coercive on the subspace $N(\Lambda)^{\perp}$, the orthogonal complement in $H$ of $N(\Lambda)$, the nullspace of $\Lambda$.

\section{§. 1 Introduction.}

This paper deals with first order matrix partial differential operators of the form

$$
\left.\left.L=-i \sum_{j=1}^{n} L_{j}^{\prime} x\right) D_{j}+L_{0} x\right)
$$

where $L_{0}(x), L_{1}(x), \ldots, L_{n}(x)$ are functions of $x=\left(x_{1}, \ldots, x_{n}\right) \in R^{n}$ whose va. lues are $m^{\prime} \times m$ matrices over the complex number field $C$, and $D_{j}=\partial / \partial x_{j}$.

(\%) This research was supported by the Office of Naval Research. Reproduction in whole or in part is permitted for any purpose of the United States Government.

(**) Entrata in Redazione il 31 luglio 1970. 
Such operators operate on functions $u(x)={ }^{t}\left(\boldsymbol{u}_{1}(x), \ldots, \boldsymbol{u}_{m}(\boldsymbol{x})\right)$ whose values are $m \times 1$ matrices $\left({ }^{1}\right)$ over $C$ to produce functions $f(x)={ }^{t}\left(f_{1}(x), \ldots, f_{m^{\prime}}(x)\right)$ whose values are $m^{\prime} \times 1$ matrices over $C$.

Thus the equation

$$
L u=f
$$

represents a system of $m^{\prime}$ first order partial differential equations for the $m$ unknown functions $u_{1}, \ldots, u_{m}$.

The set of (equivalence elasses of) $m \times 1$ matrix-valued functious $u(x)$ which are Lebesgue measurable on $R^{n}$ and satisfy ( $\left.{ }^{2}\right)$

$$
\int_{R^{n}} u(x)^{*} u(x) d x<\infty
$$

form a vector space $V_{m}$. MLoreover,

$$
(u, v)=\int_{R^{n}} u(x)^{*} v(x) d x
$$

defines an inner product on $V_{m}$ and the pair $\left(V_{m},(\cdot, \cdot)\right)$ is a Hilbert space $L_{2, m}$. More generally, let $E(x)$ be an $m \times m$ Hermiman matrix for $x \in R^{n}$ and assume that $E(x)$ is LEBRSGUE measurable and satisfies

$$
c \xi^{*} \xi \leq \xi^{*} E(x) \xi \leq c^{\prime} \xi^{*} \xi
$$

for all $\xi \in C^{m}$ and $x \in R^{n}$ where $c$ and $c^{\prime}$ are positive constants. Then

$$
(u, v)_{E}=\int_{R^{n}} u(x)^{*} E(x) v(x) d x
$$

defines an inner produet on $V_{m}$ and the pair $\left.\left(V_{m}, \therefore,\right)_{E}\right)$ is a HILBERT space $L_{2, m}(E)$. In particular $L_{2, m}=L_{2, m}(I)$ where $I$ is the unit matrix. Moreover, (1.5) implies that the norms in the spaces $L_{2, m}(E)$ are all equivalent.

Differential operators $L$ of the form (1.1) determine various unbonnded linear operators $L$ from Hilber' spaces $L_{2, m}(E)$ into HILBERT spaces $L_{2, m^{\prime}}\left(E^{\prime}\right)$. Different operators are distinguished by different choices of $E$ and $E^{\prime}$ and

(1) If $M$ is matrix then $T M$ denotes the transpose of $M$.

(2) If $M$ is a matrix then $M^{*}$ denotes the HERmitrax conjugate of $M$. Thus $M^{*}=t \bar{M}$ where $\bar{M}$ is the complex conjugate of $M$. 
the domains of definition $D(L)$. It is assumed here that $D(L)$ is a linear subspace of $L_{2, m}(E)$ such that

$$
C_{0}^{1}\left(R^{n} ; C^{m}\right) \subset D(L) \subset L_{2, n}(E)
$$

where $C_{0}^{\mathrm{l}}\left(R^{n} ; C^{m}\right)$ is the set of $m \times 1$ matrix-valued functions which are continuously differentiable in $R^{n}$ and have compact support.

Definimion 1.1. - An operator $L: L_{2, m}(E) \rightarrow L_{2, m^{\prime}}\left(E^{\prime}\right)$ is said to be coercive if and only if for all $u \in D(L), D_{j} u \in L_{2, m}(E)$ for $j=1,2, \ldots, n$, and there exists a constant $K>0$ such that for all $u \in D(L)\left({ }^{3}\right)$

$$
\sum_{j=1}^{n}\left\|D_{j} u\right\|^{2} \leq K^{2}\left(\|L u\|^{2}+\|u\|^{2}\right)
$$

Definition 1.2. - The symbol of the differential operator $L$ given by (1.1) is the $m^{\prime} \times m$ matrix-valued function

$$
L(p, x)=\sum_{j=1}^{n} L_{j}(x) p_{j}
$$

defined for $y \in R^{n}$ and $x \in R^{n}$.

Definimion 1.3. - The differential operator $L$ given by (1.1) is elliptic at $x_{0} \in h^{n}$ if and only if

$$
\operatorname{rank} L\left(p, x_{0}\right)=m \text { for all } p \in R^{n}-\{0\} \text {. }
$$

$L$ is elliptic in a region $\Omega \subset R^{n}$ if and only if it is elliptic at each $x_{0} \in \Omega$.

The concept of coerciveness for the linear operators associated with boundary value problems for partial differential equations in regions $\Omega \subset R^{n}$ was introduced by $N$. Aroxsza.s [5] and has been developed extensively (see, e.g., S. Agmon [1], D. G. de Frguerreno [11]. M. Sohechter [17], K. T. Smith [21]). In this paper the concept is used for operators on $R^{n}$ only.

The definition of ellipticity given above is a natural generulization of the definitions of ellipticity for single equations and $m \times m$ systems used in the literature (see, e.g., [3] and [10]) and of the definition of overdetermined elliptic systems given by $S$. Aamon [3]. Note that if $L$ is elliptic for some $x_{0} \in R^{n}$ then $m^{\prime} \geq m$; i.e. there are at least as many equations as unknowns.

It is known that a coercive operator is necessarily elliptic in its region of definition. Indeed, for the case considered here of operators on $R^{n}$ the

(3) The norm in (1.8) is the norm of $L_{2} \cdot{ }_{n}=L_{2},{ }_{m}(I)$. Since $L_{2},{ }_{n}(E)$ has an equivalent norm, (1.8) also holds with the norm of $L_{2},{ }_{m}(E)$. 
properties of coerciveness and ellipticity are equivalent under mild assumptions concerning the coefficient matrices. This is proved below in $\& 3$, Theorems 3.5 and 3.9. Thus a nonelliptic operator $L$ cannot be coercive in the sense of Definition 1.1. However, it is possible for it to be coercive on a subspace $V \subset L_{2, m}(E)$ in the sense of the following

Definition 1.4. - An operator $L: L_{2, m}(E) \rightarrow L_{2, m}\left(E^{\prime}\right)$ is said to be coercive on the subspace $V \subset L_{2, m}(E)$ if and only if for all $u \in D(L) \cap V$, $D_{j} u \in L_{2, m}(E)$ for $j=1,2, \ldots, n$ and there exists a constant $K>0$ such that (1.8) holds for all $u \in D(L) \cap V$.

Coercireness inequalities in this restricted sense are of great interest in connection with the study of the wave propagation problems of elassical phy. sics. It was shown in [22] that most of the wave propagation phenomena of classical physics are governed by systems of partial differential equations of the form

$$
E(x) D_{t} u+\sum_{j=1}^{n} A_{j} D_{j} u=0
$$

where $E(x)$ is an $m \times m$ Hermitran positive definite matrix for all $x \in R^{n}$, the matrices $A_{j}$ are constant $m \times m$ Hermitian matrices, $u=u(x, t)$ is an $m \times 1$ (column) matrix, $x \in L_{.}{ }^{n}$ (space) and $t \in R^{1}$ (time). Alternatively, systems (1.11) can be written in the Schrödnger form

$$
D_{\iota} u=-i \Lambda u
$$

where

$$
\Lambda=-i E(x)^{-1} \sum_{j=1}^{n} A_{j} D_{j}
$$

It is assumed here, as in [22], that $E(x)$ is bounded and uniformly positive definite on $R^{n}$; i.e. satisfies (1.5). Under these assumptions it was shown in [22] that a differential operator of the form (1.13) determines a selfadjoint operator $\Lambda$ on the HILBERT space $\mathscr{H}=L_{2, m}(E)$. The appropriate definition of $D(\Lambda)$ is given in $\$ 2$ below.

Examples of systems (1.11) include Maxwelx's equations of the electro. magnetic field, the equations of acoustics and elasticity and most of the equations of wave propagation of classical physios. It is shown below by examples that the spatial operators $\Lambda$ which arise in classical physics are usually nonelliptic.

It is the purpose of this paper to prove that the nonelliptic selfadjoint operators $\Lambda$ associated with systems of the form (1.11) are coercive on the subspaces $N(\Lambda)^{1}$, the orthocomplements in $L_{2, m}(E)$ of the nullspaces $N(\Lambda)$; 
i.e. there exists a constant $\mu>0$ such that

$$
\begin{gathered}
\sum_{j=1}^{n}\left\|D_{j} u\right\|^{2} \leq \mu^{2}\left(\|\Lambda u\|^{2}+\|u\|^{2}\right) \text { for all } \\
u \in D(\Lambda) \cap N(\Lambda)^{1} .
\end{gathered}
$$

The coerciveness inequality (1.14) is very important for theories of wave propagation and scattering governed by systems (1.11). A simple application of (1.14) is outlined below. Applications to proofs of asymptotic completeness of the wave operators and unitarity of the seattering operator for systems (1.11) will be given in separate publications [20].

A simple application of (1.14) is to the proof of regularity theorems for solutions of systems (1.11). It is known that (1.11) has a unique solution with values $u(\cdot, t) \in H$ for all initial values $u(x, 0)=u_{0}(x) \in H$; see [22, p. 47]. Moreover, it is known that if $u_{0} \in D(\Lambda)$ then $u(\cdot, t) \in D(\Lambda)$ and $D_{t} u \in H$ for all $t$. It follows that if $u_{0} \in D(\Lambda) \cap N(\Lambda)^{\perp}$ then $u(\cdot, t) \in D(\Lambda) \cap N(\Lambda)^{\perp}$ for all $t$ and hence, by (1.14), $D_{1} u(\cdot, t), \ldots, D_{n} u(\cdot, t)$ are in $H$ for all $t$. On the other hand, if $u_{0} \in D(\Lambda) \cap N(\Lambda)$ then $u(x, t)=u_{0}(x)$ for all $t$ and the regularity of $u(x, t)$ is the same as that of $u_{0}(x)$. Similar results hold for higher derivatives; cf. $\$ 6$ below.

In the remainder of this introductory section the coerciveness inequality (1.14) is proved for the special cases of electromagnetic, acoustic and elastic waves in homogeneous isotropic media. These examples provide insight into the general case and suggest the methods that are used to treat it in subsequent sections.

Example 1: Maxwell's Equations. - For the simple case of electroma. gnetic wave propagation in free space, MaxweLL's equations for the electromagnetic field can be written in the complex form

$$
D_{\imath} u=-i \nabla \times u
$$

where the complex vector $u=E+i H$ has the electric field $E$ for its real part and the magnetic field $H$ for its imaginary part. This is a Schrödingar equation (1.12) with $\Lambda_{0} u=\nabla \times u$. Hence the symbol

$$
\Lambda_{0}(p)=i\left[\begin{array}{rcr}
0 & -p_{3} & p_{2} \\
p_{3} & 0 & -p_{1} \\
-p_{2} & p_{1} & 0
\end{array}\right] \text {. }
$$

Note that $\Lambda_{0}(p)^{*}=\Lambda_{0}(p)$, i.e. $\Lambda_{0}(p)$ is Hermitian. 
The operator $\Lambda_{0}=\nabla \times$ is a simple example of a matrix partial differential operator (1.1). with $\mathrm{n}=3$ and $m^{\prime}=m=3$. Note that

$$
\text { rank } \Lambda_{0}(p)=2 \text { for all } p \in R^{3}-\{0\}
$$

Hence $\Lambda_{0}$ is not elliptic. However, note that rank $\Lambda_{0}(p)$ is a constant, independent of $p$, for all $p \neq 0$.

The coerciveness inequality for the nonelliptic operator $\Lambda_{0}=\nabla \times$ is sug. gested by the well-known Lagrange identity for vectors:

$$
|p \times v|^{2}+|p \cdot v|^{2}=|p|^{2}|v|^{2}
$$

which holds for all $p \in R^{3}$ and $v \in C^{3}$. The operators $p \times$ and $p \cdot$ can be related to the differential operators $X \nabla$ and $\nabla \cdot$ by the Plancherel theory of the Fourier transform. The transform and its inverse are defined for all $u \in L_{2, m}$ by the equations

$$
\begin{aligned}
& \widehat{u}(p)=\Phi u(p)=(2 \pi)^{-n / 2} \int_{R^{n}} e^{-i p \cdot x} u(x) d x, \\
& u(x)=\Phi^{*} \widehat{u}(x)=(2 \pi)^{-n / 2} \int_{R^{n}} e^{i x \cdot p} \widehat{u}(p) d p
\end{aligned}
$$

where the integrals converge in $L_{2, m}$ (but not necessarily pointwise).

The transform maps $L_{2, m}$ onto itself and is an isometry; i.e. PARSEval's theorem

$$
\|\widehat{u}\|=\|u\|
$$

holds for all $u \in L_{2, m}$.

Define the domains of the operators $\nabla \times$ and $\nabla \cdot$ by

$$
\begin{aligned}
& D(\nabla \times)=L_{2,3} \cap\left\{u: \nabla \times u \in L_{2,3}\right\}, \\
& D(\nabla \cdot)=L_{2,3} \cap\left\{u: \nabla \cdot u \in L_{2,3}\right\}
\end{aligned}
$$

Then take $v=\widehat{u}(p)$ in (1.18) and integrate the identity over $p \in R^{3}$.

Then the Prancheres theory implies that $u \in D(\nabla \times) \cap D(\nabla \cdot)$ if and only if $u, D_{1} u, D_{2} u$ and $D_{3} u$ are in $L_{2,3}$ and the identity

$$
\|\nabla \times u\|^{2}+\|\nabla \cdot u\|^{2}=\sum_{j=1}^{3}\left\|D_{j} u\right\|^{2}
$$

bolds. In particular, $\nabla \times$ is coercive on the subspace $N(\nabla \cdot)$, the nullspace of the divergence operator. 
J. R. Schulenberger - C. H. Wilcox: Coerciveness inequalities, etc. 235

Identity (1.22) snggests the introluction of the $1 \times 3$ matrix operator $\Lambda_{0}^{\prime} u=\nabla \cdot u$. Clearly

$$
\Lambda_{0}^{\prime}(p)=i\left(p_{1} p_{2} p_{3}\right)
$$

and rank $\Lambda_{0}^{\prime}(p)=1$ for $p \neq 0$. Consider the augmented $4 \times 3$ matrix operator $\Lambda_{0}^{\prime \prime}$ with symbol

$$
\Lambda_{0}^{\prime \prime}(p)=\left(\begin{array}{c}
\Lambda_{0}^{\prime}(p) \\
\Lambda_{0}(p)
\end{array}\right)
$$

Lagrange's identity (1.18) can be written

$$
\left.\left|\Lambda_{0}^{\prime \prime}(p) v^{2}=\right| \Lambda_{0}^{\prime}(p) v\right|^{2}+\left|\Lambda_{0}(p) v\right|^{2}=|p|^{2}|v|^{2} .
$$

Thus $\Lambda_{0}^{\prime \prime}$ is elliptic and $(1.22)$ is equivalent to the identity

$$
\left\|\Lambda_{0}^{\prime \prime} u\right\|^{2}=\left\|\Lambda_{0}^{\prime} u\right\|^{2}+\left\|\Lambda_{0} u\right\|^{2}=\sum_{j=\mathrm{i}}^{3}\left\|D_{j} u\right\|^{2}
$$

for all $u \in D\left(\Lambda_{0}^{\prime \prime}\right)=D\left(\Lambda_{0}^{\prime}\right) \cap D\left(\Lambda_{0}\right)$, which implies that $\Lambda_{0}^{\prime \prime}$ is coercive.

The operator $\Lambda_{0}$ is elosed and densely defined on $L_{2,3}$ (see, e.g. [14] for definitions). Hence

$$
R\left(\Lambda_{0}\right)^{\perp}=N\left(\Lambda_{0}^{*}\right)
$$

i.e. the orthogonal complement of the range of $\Lambda_{0}$ in $L_{2,3}$ is the null space of $\Lambda_{0}^{*}$, the adjoint of $\Lambda_{0}$ on $L_{2,3}\left[14\right.$, p. 267]. Since $\Lambda_{0}^{*}=\Lambda_{0}$ this implies $R\left(\Lambda_{0}\right)^{\perp}=N\left(\Lambda_{0}\right)$ and hence $\bar{R}\left(\overline{\left.\Lambda_{0}\right)}=N\left(\Lambda_{0}\right)^{ \pm}\right.$, where the bar denotes closure in $L_{2,3}$.

The symbols $\Lambda_{0}^{\prime}(p)$ and $\Lambda_{0}(p)$ satisfy the identities

$$
\Lambda_{0}^{\prime}(p) \Lambda_{0}(p)=\Lambda_{0}^{\prime}(p) \Lambda_{0}(p)^{*}=0
$$

which imply that

$$
\Lambda_{0}^{\prime} \Lambda_{0}=0
$$

i.e. $u \in D\left(\Lambda_{0}\right)$ implies $v=\Lambda_{0} u \in D\left(\Lambda_{0}^{\prime}\right)$ and $\Lambda_{0}^{\prime} v=0$. Equation (1.29) is equivalent to the well-known identity «div eurl $=0$ \%. It implies that

$$
N\left(\Lambda_{0}\right)^{1}=R\left(\overline{\left.\Lambda_{0}\right)} \subset N\left(\Lambda_{0}^{\prime}\right) .\right.
$$


Thus (1.26) implies that $\Lambda_{0}=\nabla \times$ is coercive on $N\left(\Lambda_{0}\right)^{\perp}$, the orthocomplement of its nullspace in $L_{2,3}$ : in fact

$$
\left\|\Lambda_{0} u\right\|^{2}=\sum_{j=1}^{3}\left\|D_{j} u\right\|^{2} \quad \text { for all } u \in D\left(\Lambda_{0}\right) \cap N\left(\Lambda_{0}\right)^{\perp},
$$

which implies (1.14) with $K=1$.

Notice that the identity $\left.(1.28), \Lambda_{0}^{\prime} p\right) \Lambda_{0}(p)^{*}=0$, implies that the rows of $\Lambda_{0}^{\prime}(p)$ and $\Lambda_{0}(p)$ are orthogonal vectors in the sense of the Henmitian inner product $(\xi, \eta)=\sum_{j=1}^{m} \xi_{j} \bar{\eta}_{j}$. Hence, the rows of $\Lambda_{0}^{\prime}(p)$ and $\Lambda_{0}(p)$ are linearly independent and the conditions rank $\Lambda_{0}^{\prime}(p)=1$ and rank $\Lambda_{0}^{\prime}(p)=2$ for all $p \neq 0$ imply that rank $\Lambda_{0}^{\prime \prime}(p)=3$, i.e. the augmented operator $\Lambda_{0}^{\prime \prime}$ is elliptic. It is (1.28) rather than (1.18) that is extended below to more general operators $\Lambda_{0}$.

Example 2: The Equations of Acoustics. - Two-dimensional acoustic waves in a homogeneous fluid can be described by solutions of a Schrödinger equation (1.10) with

$$
\Lambda_{0}(p)=\left[\begin{array}{llr}
0 & 0 & p_{1} \\
0 & 0 & p_{2} \\
p_{1} & p_{2} & 0
\end{array}\right]
$$

(See $\left[22\right.$, p. 75] for a derivation). In this example $n=2$ and $m^{\prime}=m=3$. Note that $\Lambda_{0}(p)$ is real symmetric and hence $\Lambda_{0}(p)^{*}=\Lambda_{0}(p)$. Moreover,

$$
\text { rank } \Lambda_{0}(p)=2 \text { for all } p \in R^{2}-\{0\} \text {, }
$$

whence $\Lambda_{0}$ is not elliptic.

A left null vector for $\Lambda_{0}(p)$ is

$$
\Lambda_{0}^{\prime}(p)=\left(p_{2},-p_{1} 0\right) .
$$

This suggests consideration of the augmented operator $\Lambda_{0}^{\prime \prime}$ with symbol given by $(1.24)$ in which $\left.\Lambda_{0}^{\prime} p\right)$ is defined by (1.32) and $\Lambda_{0}^{\prime}(p)$ by (1.34). A sim. ple computation shows that identity (1.25) also holds in this case. Thus $\Lambda_{0}^{\prime \prime}$ is elliptic and satisfies the identity (1.26). Finally, by the same arguments as for MAXwell's equations, $\Lambda_{0}$ is coereive on the orthocomplement of its nullspace in $L_{2,3}$ and (1.31) holds.

The two examples given above of wave propagation systems (1.11) are very special because the associated operator $\Lambda_{0}$ given by (1.13) can be ang. 
J. R. Schulenberger - C. H. Wrlcox: Coerciveness inequalities, etc. 237

mented by a first-order operator $\Lambda_{0}^{\prime}$ in such a way that $\Lambda_{0}^{\prime \prime}$ is elliptic. This is because electromagnetic and acoustic waves propagate with a single nonzero speed. For more general systems (1.11) there will be several propagation speeds (varying with $p$ if the medinm is anisotropic). The situation is illustrated by the example of two-dimensional elastic waves in a homogeneous isotropic solid for which there are two nontero speeds, corresponding to pressure and shear waves.

EXample 3: The Equations of Elasticity. - Two-dimensional elastic waves in a homogeneous isotropic solid are described by a displacement vector $w=w(x, t)=w_{1}(x, t) \vec{i}+w_{2}(x, t) \vec{j}$ which satisfies the second order vector equation [15]

$$
D_{t}^{2} w=\mu \Delta w+(\lambda+\mu) \nabla \nabla \cdot w
$$

where $x=\left(x_{1}, x_{2}\right) \in R^{2}$ and $\lambda$ and $\mu$ are positive constants. The components $\Sigma_{i j}$ of the stress tensor are related to the dispacement by [15]

$$
\begin{aligned}
& \Sigma_{11}=(\lambda+2 \mu) D_{1} w_{1}+\lambda D_{2} w_{2} \\
& \Sigma_{12}=\Sigma_{21}=\mu\left(D_{1} w_{2}+D_{2} w_{1}\right) \\
& \Sigma_{22}=(\lambda+2 \mu) D_{2} w_{2}+D_{1} w_{1}
\end{aligned}
$$

and the velocity is given by

$$
v_{1}=D_{\imath} w_{1}, v_{2}=D_{\imath} w_{2} .
$$

Introduce now dependent variables $u={ }^{t}\left(\Sigma_{11}, \Sigma_{22}, \Sigma_{12}, v_{1}, v_{2}\right)$. Then equations (1.35) through (1.37) imply that $u$ is a solution of a first-order system (1.11). The energy density for (1.35) is [15]

$$
\eta=2^{-1}\left[(\lambda+2 \mu)\left(\sigma_{11}^{2}+\sigma_{22}^{2}\right)+2 \lambda \sigma_{11} \sigma_{22}+4 \mu \sigma_{12}^{2}+v_{1}^{2}+v_{2}^{2}\right]
$$

where

$$
\sigma_{i k}=2^{-1}\left(D_{j} w_{k}+D_{k} w_{j}\right)
$$

When expressed in terms of the components of $u$, this takes the form

$$
\eta=2^{-1}\left[a u_{1}^{2}+2 b u_{1} u_{2}+a u_{2}^{2}+(1 / \mu) u_{3}^{2}+u_{4}^{2}+u_{5}^{2}\right]
$$

where

$$
a=(\lambda+2 \mu) /[4 \mu(\lambda+\mu)], \quad b=-\lambda /[4 \mu(\lambda+\mu)] .
$$


It follows that $u$ satisfies an equation of the form (1.11) with

and

$$
E_{0}=\left[\begin{array}{ccccc}
a & b & 0 & 0 & 0 \\
b & a & 0 & 0 & 0 \\
0 & 0 & 1 / \mu & 0 & 0 \\
0 & 0 & 0 & 1 & 0 \\
0 & 0 & 0 & 0 & 1
\end{array} \mid\right.
$$

$$
A(p)=\sum_{j=1}^{2} A_{j} p_{j}=\left[\begin{array}{ccccc}
0 & 0 & 0 & -p_{1} & 0 \\
0 & 0 & 0 & 0 & -p_{2} \\
0 & 0 & 0 & -p_{2} & -p_{1} \\
-p_{1} & 0 & -p_{2} & 0 & 0 \\
0 & -p_{2}-p_{1} & 0 & 0
\end{array}\right]
$$

The operator $\Lambda_{0}$ of $(1.13)$ has the symbol $\Lambda_{0}(p)=E_{0}^{-1} A(p)$. It is an example of a system (1.1) with $n=2$ and $m^{\prime}=m=5$. It is easy to verify that

$$
\operatorname{rank} \Lambda_{0}(p)=\operatorname{rank} A(p)=4 \text { for all } p \in R^{2}-\{0\},
$$

whence $\Lambda_{0}$ is not elliptic.

A left null vector for $A(p)$ is

$$
A^{\prime}(p)=\left(\begin{array}{llll}
p_{2}^{2} & p_{1}^{2}-p_{1} p_{2} & 0 & 0
\end{array}\right)
$$

Thas if

$$
\Lambda_{0}^{\prime}(p)=A^{\prime}(p) E_{0}^{\prime}
$$

then $\Lambda_{0}^{\prime}(p) \Lambda_{0}(p)=0$ and also

$$
\left.\Lambda_{0}^{\prime}(p) E_{0}^{-1} \Lambda_{0}{ }^{\prime} p\right)^{*}=A^{\prime}(p) A(p) E_{0}^{-1}=0 .
$$

The last equation implies that the rows of $\Lambda_{0}^{\prime}(p)$ and $\Lambda_{0}(p)$ are orthogonal with respect to the inner product $(\xi, \eta)=\xi E_{0}^{-1-} \eta$. If follows that the augmented form

$$
\left.\Lambda_{0}^{\prime \prime \prime} p\right)=\left(\begin{array}{c}
\Lambda_{0}^{\prime}(p) \\
\Lambda_{0}(p)
\end{array}\right)
$$

has $\operatorname{rank} \Lambda_{0}^{\prime \prime}(p)=5$. 
In the present case it is easy to verify that there is no first-order ope. rator $\Lambda_{0}^{\prime}$ such that $\Lambda_{0}^{\prime \prime}$ is elliptic. Accordingly the coerciveness of $\Lambda_{0}$ on the orthocomplement of its nullspace is more difficult to prove. Several alternatives present themselves. One is to keep the definitions (1.45), (1.46), (1.48) above and work with the concept of ellipticity due to A. Dougris and L. Nirenberg [9] where the equations and unknowns are allowed to have different orders. A second alternative is to define

$$
\Lambda_{0}^{\prime \prime}(p)=\left(\begin{array}{c}
\Lambda_{0}^{\prime}(p) \\
\Lambda_{0}(p)^{2}
\end{array}\right)
$$

where $\Lambda_{0}^{\prime}(p)$ is given by (1.45), (1.46) and use the theory of elliptic systems of the second order. This approach gives the coerciveness of $\Lambda_{0}^{2}$ on $N\left(\Lambda_{0}\right)$. A third alternative is to given up the requirement that $\Lambda_{0}^{\prime}$ be a differential operator and define

$$
\Lambda_{0}^{\prime}(p)=|p|\left(\eta_{2}^{2}-\eta_{1}^{2}-\eta_{1} \eta_{2} \quad 0 \quad 0\right) E_{0}, \quad \eta=p / p \mid
$$

The corresponding operator can be written

$$
\Delta_{0}^{\prime}=(-\Delta)^{1 / 2}\left(-D_{2}^{2}-D_{1}^{2} D_{1} D_{2} \quad 0 \quad 0\right) E_{0}
$$

It is the third method that is followed here and for more general systems in $\S 5$ below. In the present case notice that since $\Lambda_{0}^{\prime \prime}(p)$ has rank 5 for all $p \in R^{2}-\{0\}$, there exists a positive constant $\mu$ such that

$$
\left|\Lambda_{0}^{\prime \prime}(\eta) \tilde{\mu}\right| \geq \mu^{2}>0
$$

for all unit vectors $\eta$ and $\tilde{u}$. Now $\Lambda_{o}^{\prime \prime}(p)$ is homogeneous of degree one if $\Lambda_{0}^{\prime}(p)$ is defined by (1.50). Thas putting $\eta_{i}=p /|p|$ and $\tilde{u}=\widehat{u}(p) /|\widehat{u}(p)|$ in (1.52) gives

$$
\left|\Lambda_{0}^{\prime \prime}(p) \widehat{u}(p)\right|^{2} \geq \mu^{2}|p|^{2}|\widehat{u}(p)|^{2} \quad \text { for all } p \in R^{2}
$$

and all $u \in L_{2,5}$. Since $E_{0}$ is constant it is natural to define

$$
D\left(\Lambda_{0}\right)=L_{2,5}\left(E_{0}\right) \cap\left\{u: \Lambda_{0}(p) \bar{u}(p) \in L_{2,5}\left(E_{0}\right)\right\} \text { and }
$$

$$
D\left(\Lambda_{0}^{\prime}\right)=L_{2,5}\left(E_{0}\right) \cap\left\{u: \Lambda^{\prime}(p) \widehat{\boldsymbol{u}}(p) \in L_{2,1}\right\}
$$


and $D\left(\Lambda_{0}^{\prime \prime}\right)=D\left(\Lambda_{0}\right) \cap\left(D \Lambda_{0}^{\prime}\right)$. With these definitions, (1.53) and the Prancherel theory imply that

$$
\left\|\Lambda_{0}^{\prime \prime} u\right\|^{2}=\left\|\Lambda_{0} u\right\|^{2}+\left\|\Lambda_{0}^{\prime} u\right\|^{2} \geq \mu^{2} \sum_{j=1}^{2}\left\|D_{j} u\right\|^{2}
$$

for all $u \in D\left(\Lambda_{0}^{\prime \prime}\right)$.

The operator $\Lambda_{0}$ is selfadjoint on the HILbert space $L_{2,5}\left(E_{0}\right)$. Hence $\overline{R\left(\Lambda_{0}\right)}=N\left(\Lambda_{0}\right)^{\perp}$, where the orthocomplement is taken in $L_{2,5}\left(E_{0}\right)$. Moreover,

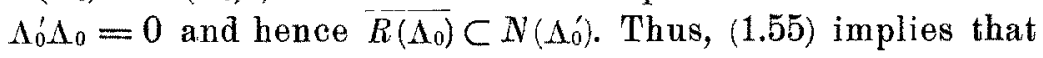

$$
\sum_{j=1}^{2}\left\|D_{j} u\right\|^{2} \leq \mu^{-2}\left\|\Lambda_{0} u\right\|^{2} \quad \text { for all } u \in D\left(\Lambda_{0}\right) \cap N\left(\Lambda_{0}\right)^{1}
$$

Hence $\Lambda_{0}$ is coercive on the orthocomplement of its nullspace in $L_{2,5}\left(E_{0}\right)$.

The operator $\Lambda_{0}$ for each of the three examples has the property that rank $\Lambda_{0}(p)$ is constant, independent of $p \in R^{n}-\{0\}$. This suggests

Definimion 1.5. - The differential operator $L$ given by (1.1) has constant deficit $k$ at $x_{0} \in R^{n}(1 \leq k \leq m)$ if and only if

$$
\operatorname{rank} L\left(p, x_{0}\right)=m-k \text { for all } p \in R^{n}-\{0\} .
$$

$L$ has constant deficit $k$ in a region $\Omega \subset R^{n}$ if and only if (1.57) holds for each $x_{0} \in \Omega$.

For operators

$$
\Lambda_{0}=-i E_{0}^{-1} \sum_{j=1}^{n} A_{j} D_{j}
$$

with constant coefficients and constant deficit the coerciveness inequality (1.14) can be proved via the Plancherel theory in the same way as for the equations of elasticity. A proof is given in $\$ 5$ below.

The class of uniformly propagative systems

$$
E_{0} D_{t} u+\sum_{j=1}^{n} A_{j} D_{j} u=0
$$

was introduced in [22]. In essence, these are characterized by the property that the roots $\lambda_{j}(p)$ of the characteristic polynomial

$$
P(p, \lambda)=\operatorname{det}\left(E_{0} \lambda-\sum_{j=1}^{n} A_{j} p_{j}\right)
$$

have constant algebraic sign and constant multiplicity for all $p \in R^{n}-\{0\}$. In particular, a root $\lambda_{j}(p)=0$ if and only if it is identically zero and the 
root $\lambda_{j}(p) \equiv 0$ (if there is one) has constant multiplicity $k$ for all $p \in R^{n}-\{0$. It follows that the operator $\Lambda_{0}$ for a uniformly propagative system has constant deficit $k$.

For operators $\Lambda$ with variable coefficients the FourIER transform method fails and the proof of coerciveness inequalities is much more complicated, just as in the case of GARDING's inequality and coerciveness inequalities for elliptic operators.

The remainder of this paper is organized as follows. In $\& 2$ the linear operators from $L_{2, m}(E)$ into $L_{2 . m^{\prime}}\left(E^{\prime}\right)$ determined by a differential operator $L$ of the form (1.1) are discussed. It is shown that $L$, restricted to $C_{0}^{1}\left(R^{n} ; C^{m}\right)$, has a unique extension to a closed operator from $L_{2 . m}(E)$ into $L_{2, m^{\prime}}\left(E^{\prime}\right)$. In $\S 3$ coerciveness inequalities for elliptic systems are discussed. In $\& 4$ the basic construction of an elliptic augmentation $L^{\prime \prime}$ for a nonelliptic operator $L$ of constant deficit is given, and a coerciveness theorem is proved for the case where $L^{\prime \prime}$ is a first-order operator. Sections 5 and 6 contain the principal new results in the paper. In $\S 5$ it is proved that a class of operators $\Lambda$ of the form (1.13) which are perturbations of uniformly propagative systems $\Lambda_{0}$ of the form (1.58) are coercive on $N(\Lambda)^{\perp}$. In $\S 6$ coerciveness inequalities for higher order derilatives are proved for perturbations of uniformly propagative systems. Section 7 contains a brief discussion of unsolved problems concerning coerciveness inequalities for nonelliptic operators.

\section{\& 2. - Operators on Hilbert Spaces Determined by a Partial Differential Operator.}

This preparatory section contains a discussion of the various linear operators between the Bilbert spaces $L_{2, m}(E)$ and $L_{2 . m^{\prime}}\left(E^{\prime}\right)$ determined by a first-order differential operator $L$ of the form (1.1). The main result states that, under mild assumptions concerning the coefficients, the restriction of $L$ to $C_{0}^{l}\left(R^{n} ; C^{m}\right)$ has a unique extension to a closed linear operator from $L_{2, m}(E)$ to $L_{2, m^{\prime}}\left(E^{\prime}\right)$. This is a consequence of K. O. Friedriohs' famous theo. rem [12] concerning the identity of weak and strong extensions of differential operators.

In this section the following assumptions are made concerning the coefficients of the differential operator $L$ :

$$
\begin{aligned}
& \left.L_{j}{ }^{\prime} x\right) \text { and } D_{k} L_{j}(x) \text { are continuous and bounded on } R^{n} \\
& \text { for } 1 \leq j, k \leq n ;
\end{aligned}
$$

$L_{0}(x)$ is continuous and bounded on $R^{n}$. 
Some of the results given below can be proved under weaker assump. tions then these. However, for simplicity assumptions (2.1) and (2.2) are re. tained throughout the paper.

A differential operator $L$ can be used to define various linear operators from $L_{2, m}(E)$ to $L_{2, m^{\prime}}(E)$. Three of these are considered here, namely the minimal operator and its strong and weak extensions.

Definimion 2.1. - The minimal operator for $L$ is the operator $\tilde{L}: L_{2, m}(E) \rightarrow$ $\rightarrow L_{2, m^{\prime}}\left(E^{\prime}\right)$ with domain

$$
D(\tilde{L})=C_{0}^{1}\left(R^{n} ; C^{m}\right)
$$

whose action is defined by

$$
\tilde{L} u=L u=-i \sum_{j=1}^{n} L_{j}(x) D_{j} u+L_{0}(x) u \text { for all } u \in D(\tilde{L})
$$

Clearly, if $E(x)$ and $E^{\prime}(x)$ satisfy (1.5) and $L$ satisfies (2.1) and (2.2) then $\tilde{L} u \in L_{2 . m^{\prime}}\left(E^{\prime}\right)$ for all $u \in D(\tilde{L})$. Hence $\tilde{L}$ is a well-defined linear operator from $L_{2, m}(E)$ to $L_{2, m^{\prime}}\left(E^{\prime}\right)$.

The definitions of the strong and weak extensions of $\tilde{L}: L_{2, n}(E) \rightarrow L_{2, m^{\prime}}(E)$ make use of a differential operator $L_{E, E^{\prime}}^{*}$, the formal adjoint of $L$ with re. spect to the inner products $(u, v)_{E}$ and $\left(u^{\prime}, v^{\prime}\right)_{E^{r}}$ which is defined by the identity

$$
(L u, v)_{E^{\prime}}=\left(u, L_{E, E^{\prime}}^{+} v\right)_{E}
$$

for all $u \in C_{0}^{1}\left(R^{n} ; C^{m}\right)$ and $v \in C_{0}^{1}\left(R^{n} ; C^{m^{\prime}}\right)$. A detailed discussion of the special case where $E=I, E^{\prime}=I^{\prime}$ is given below. Then the extension to more general $E$ and $E^{\prime}$ is indicated and an application is given to the operators $\Delta$ that arise in the theory of wave propagation.

The simplified notation $L^{\dagger}=L_{I, L^{\prime}}^{\dagger}$ will be used in what follows. With this convention integration by parts gives

$$
(L u, v)=\left(u, L^{+} v\right)
$$

for all $u \in C_{0}^{1}\left(R^{n} ; C^{m}\right)$ and $v \in C_{0}^{1}\left(R^{n} ; C^{m^{\prime}}\right)$ where

$$
L^{+} v=-i \sum_{j=1}^{n} D_{j}\left\{L_{j}^{*} v\right\}+L_{0}^{*} v .
$$


Note that under hypotheses (2.1) and (2.2) $L^{\dagger}$ is an $m \times m^{\prime}$ matrix differential operator of the same form as $L$ :

$$
L^{+}=-i \sum_{j=1}^{n} L_{j}^{*}(x) D_{j}+\left(L_{0}^{*}(x)-i \sum_{j=1}^{n} D_{j} L_{j}^{*}(x)\right)
$$

Moreover, $L^{i+}=\left(L^{+}\right)^{+}=L$.

Note that $(2.6)$ is equivalent to

$$
(\tilde{L} u, v)=\left(u, \tilde{L}^{+} v\right)
$$

for all $u \in D(\tilde{L})$ and $v \in D\left(\tilde{L}^{+}\right)$. It follows that

$$
\tilde{L}^{+} \subset \tilde{L}^{*}
$$

where $\tilde{L}^{*}$ denotes the adjoint of the HxLBERT space operator $\tilde{L}: L_{2, m} \rightarrow L_{2, m^{\prime}}$. In particular, $\tilde{L}^{*}$ is densely defined on $L_{2, m^{\prime}}$ since $C_{0}^{1}\left(R^{n} ; C^{m^{\prime}}\right)=D\left(L^{+}\right)$is dense in $L_{2, m^{\prime}}$. It follows that $\tilde{L}$ is a closable operator whose closure is the operator $\tilde{L}^{* *}[14$, p. 267$]$.

Definturon 2.2. - The strong extension $L_{s}: L_{2, m} \rightarrow L_{2, m^{\prime}}$ of the minimal operator $\tilde{L}: L_{2, m} \rightarrow L_{2 . m^{\prime}}$ is the closure of $\tilde{L}$; i.e.

$$
L_{s}=\tilde{L}^{* *} \text {. }
$$

Thus $u \in D\left(L_{s}\right)$ and $L_{s} u=v$ if and only if there exists a sequence $\left\{u_{k}\right\} \subset D(\tilde{L})$ such that $u_{k} \rightarrow u$ in $L_{2, m}$ and $\tilde{L} u_{k} \rightarrow v$ in $L_{2, m^{\prime}}$.

Definxtion 2.3. - The weak extension $L_{w}$ of the minimal operator $\tilde{L}: L_{2, m} \rightarrow L_{2, m^{\prime}}$ is the operator $L_{w}: L_{2, m} \rightarrow L_{2, m^{\prime}}$ defined by the conditions hat $u \in D\left(L_{w}\right)$ and $L_{w v} u=v$ if and only if $v \in L_{2, m^{\prime}}$ and

$$
\left(u, \tilde{L}^{\dagger} \varphi\right)=(v, \varphi) \text { for all } \varphi \in C_{0}^{1}\left(R^{n} ; C^{m^{\prime}}\right)=D\left(\tilde{L}^{\dagger}\right) .
$$

Each $u \in D\left(L_{v e}\right)$ has a unique image $v=L_{v} u$ because $C_{0}^{\mathbf{l}}\left(R^{n} ; C^{m^{\prime}}\right)$ is dense in $L_{2, m^{\prime}}$. Moreover, (2.9) implies that

$$
\tilde{L} \subset L_{w}
$$

i.e. $L_{w}$ is indeed an extension of $\tilde{L}$. Moreover, it is obvious from the defini. tion that $L_{w}$ is a closed operator. Hence (2.13) implies

$$
L_{s} \subset L_{w} \text {. }
$$


Note that Definition 2.3 is equivalent to defining $L_{z v}$ to be the HrLBertspace adjoint of $\tilde{L}^{+}$, the minimal operator for $L^{+}$:

$$
L_{w}=\left(\tilde{L}^{\dagger}\right)^{*}
$$

It is a remarkable fact, discovered by K. 0 . Friedrichs [12], that the weak and strong extensions coincide for first-order operators whose coeffi* cients satisfy (2.1) and (2.2).

Theorem 21 . (Friedrichs' Theorem). - If $L$ is a differential operator whose coefficients satisfy $(2.1)$ and $(2.2)$ then

$$
L_{s}=L_{w}
$$

$L_{s}$, the closure of $\tilde{L}$, is clearly the minimal closed extension of $\tilde{L}$. Moreover, $D\left(L_{w}\right)$ can be described in terms of distribution theory as the set of all distributions $u \in L_{2, m}$ for which the distribution $L u$ is in $L_{2, m^{\prime}}$. Thus $L_{w}$ is the maximal closed extension of $\tilde{L}$. Hence, Theorem 2.1 implies that if $L$ satisfies (2.1) and (2.2) then the minimal operator $\tilde{L}: L_{2, m} \rightarrow L_{2, m^{\prime}}$ has a unique closed extension $L_{s}=L_{w}$.

Proof of Theorem 2.1. - The proof is, in essence, contained in Frit: DRICHs' original paper. Hence it is only sketched here.

Because of (2.14) it suffices to show that $L_{w} \subset L_{s}$. For this it is enough to show that if $u \in D\left(L_{w}\right)$ then there exists a sequence $\left\{u_{k} ! \subset D(\tilde{L})=C_{0}^{1}\left(R^{n} ; C^{m}\right)\right.$ such that $u_{k} \rightarrow u$ in $L_{2, m}$ and $\tilde{L} u_{k} \rightarrow L_{w} u$ in $L_{2, m^{\prime}}$. The approximation is ear. ried out in two steps. First, let $\psi(t) \in C^{1}(R), 0 \leq \psi(t) \leq 1, \psi(t) \equiv 1$ for $t \leq 0$ and $\psi(t) \equiv 0$ for $t \geq 1$ and define $\psi_{R}(x)=\psi(|x|-R)$. Then it is easy to check that if $u \in D\left(L_{w}\right)$ then $u_{R}=\psi_{R} u \in D\left(L_{w}\right), u_{R} \rightarrow u$ in $L_{2, m}$ and $L_{w} u_{R} \rightarrow L_{w} u$ in $L_{2, m^{\prime}}$ when $R \rightarrow \infty$. Hence, it suffices to construct an approximating sequence $\left\{u_{k}{ }^{\prime} \subset D(\tilde{L})\right.$ for vectors $u \in D\left(L_{w}\right)$ with compact support.

To construct the sequence $\left\{\boldsymbol{u}_{k}\right\}$, FRIEDRIOHs introduced his well-known «mollifier», as follows. Let $j(t) \in C_{0}^{1}(R), j(t) \equiv 0$ for $|t| \geq 1, j(t) \geq 0$ and

$$
\int_{-1}^{1} j(t) d t=1
$$

Define

$$
j_{k}(x)=k^{n} j\left(k x_{1}\right) j\left(h x_{2}\right) \ldots j\left(k x_{n}\right)
$$

and define the sequence $\left|u_{k}\right|$ and operator $J_{k}$ by

$$
\left.u_{k}(x)=J_{k} u(x)=\int_{R^{n}} j_{k}^{\prime}, x-y\right) u^{\prime} y d y .
$$


The basic approximation result for $J_{k}$ is (see [12] and [13] for proofs).

Lemma 2.3. - The operator $J_{k}$ is bounded on $L_{2, m}$ with bound $\left\|J_{k}\right\| \leq 1$ for all $k$. Moreover, for all $u \in L_{2, m}$,

$$
\lim _{k \rightarrow \infty} u_{k}=u \text { in } L_{2, m}
$$

If $u \in D\left(L_{w}\right)$ and has compact support then clearly $u_{k} \in C_{0}^{1}\left(R^{n} ; C^{m}\right)$ and $u_{k} \rightarrow u$ in $L_{2, m}$ by Lemma 2.3. Thus, the proof of Theorem 2.1 will be complete if it is shown that $\tilde{L} u_{k} \rightarrow L_{w} u$ in $L_{2, m^{2}}$. Now

$$
\tilde{L} \boldsymbol{u}_{k}=\tilde{L} J_{k} u=J_{k} L_{w} u+\left(\tilde{L} J_{k}-J_{k} L_{w}\right) \boldsymbol{u} .
$$

and $J_{k} L_{w w} u \rightarrow L_{s w} u$ by Lemma 2.3. Hence it suffices to show that the last term in (2.21) tends to zero in $L_{2, m^{\prime}}$ when $k \rightarrow \infty$. Friedrichs showed that the operator $\tilde{L}_{J_{k}}-J_{k} L_{w}$ can be extended to be a bounded operator from $L_{2, m}$ to $L_{2, m^{\prime}}$ (in fact, an integral operator) and that

$$
\lim _{k \rightarrow \infty}\left\|\tilde{L} J_{k}-J_{k} L_{w}\right\|=0
$$

when the coefficients of $L$ satisfy (2.1) and (2.2). This completes the sketch of the proof of Theorem 2.1.

In the remainder of this paper, if $L$ is a first-order partial differential operator whose coefficients satisfy $(2.1)$ and $(2.2)$ then the closed linear ope. rator $L_{s}=L_{w}$ will also be denoted by $L$. In each case it will be clear from the context which meaning is intended.

A corollary of Friedrichs' theorem that is needed below in $\& 5$ is

Coroluary 2.2. - Let $I$, be a differential operator whose coefficients satisfy (2.1) and (2.2) and let $L=L_{s}=L_{w}$ denote the unique closed extension of $L$. Then if $u \in D(L)$ also $J_{k} u \in D(L)$ and $\lim _{k \rightarrow \infty} L_{k} J_{k} u=L u$ in $L_{2, m^{\prime}}$.

Proof. - Let $u \in D(L)$ and let $\left\{u_{j} \backslash \subset C_{0}^{1}\left(R^{n} ; C^{m}\right)\right.$ be such that $u_{j} \rightarrow u$ and $L u_{j} \rightarrow L u$ in $L_{2, m}$ when $j \rightarrow \infty$. This is possible because $L=L_{s}$ is the closure of $\tilde{L}$. Then $L J_{k} u_{j}=J_{k} L u_{j}+\left(L J_{k}-J_{k} L\right) u_{j}=J_{k} L u_{j}+\left(\tilde{L} J_{k}-J_{k} L\right) u_{j}$. Now $J_{k}$ and $L J_{k}-J_{k} L$ are bounded operators on $L_{2, m}$ for fixed $k$. Thus $\left\{L J_{k} u_{j}\right\}$ is a CAUCHY sequence in $L_{2, n}$ for $j \rightarrow \infty$. Moreover, $J_{k} u_{j} \rightarrow J_{k} u$ for $j \rightarrow \infty$. Thus, since $L$ is a closed operator, $J_{k} u \subset D(L)$ and $L J_{k} u_{j}=\tilde{L} J_{k} z_{j} \rightarrow L J_{k} u$ in $L_{2, m}$. This implies that if $R_{k}$ is the bounded extension of $\tilde{l}_{.} J_{k}-J_{k} L$ on $L_{2, m}$ then the restriction of $K_{k}$ to $D(L)$ is $L J_{k}-J_{k} L$. To show that $L J_{k} u \rightarrow L u$ when $k \rightarrow \infty$, note that since $J_{k} u \in D(L), L u-L J_{k} u=\left(L u-L \Psi_{R} u\right)+\left(L \psi_{R} u-L J_{k} \psi_{R} u\right)+$ $+\left(L J_{k} \psi_{R} u-J_{k} L \psi_{R} u\right)+\left(J_{k} L \psi_{R} u-J_{k} L u\right)+\left(J_{k} L u-L J_{k} u\right)=\left(L u-L \psi_{R} u\right)+\left(L \psi_{R} u-\right.$ $\left.-L J_{k} \psi_{R} u\right)+\left(I_{L} J_{k}-J_{k} L\right)\left(\psi_{R} u-u\right)+J_{k}\left(L \psi_{R} u-L u\right)$ where $\psi_{R}(x)=\psi(|x|-R)$ is 
the function defined in the proof of Theorem 2.1. Let $\left\|L J_{k}-J_{k} L\right\| \leq M$, where $M$ is independent of $k$. (This is possible by (2.22)). Then given any $\varepsilon>0$ choose $R$ such that $\left\|L u-L \psi_{R} u\right\|<\varepsilon / 4$ and $\left\|\psi_{R} u-u\right\| \leq \varepsilon / 4 M$. ( $R$ does not depend on $k$ ). Then $\left\|L u-L J_{k} u\right\| \leq \varepsilon / 4+\left\|L \psi_{R} u-L J_{k} \psi_{R} u\right\|+\varepsilon / 4+\varepsilon / 4=$ $=3 \varepsilon / 4+\left\|L \psi_{R} u-L J_{k} \psi_{R} u\right\|$. The last term tends to zero (for fixed $R$ ) when $k \rightarrow \infty$ by the proof of Theorem 2.1 (2.21) and the argument following it).

The strong and weak extensions of the minimal operator $\tilde{L}: L_{2, m}(E) \rightarrow L_{2 . m^{\prime}}\left(E^{\prime}\right)$ can be defined by means of the formal adjoint $L_{E, E^{\prime}}^{+}$in strict analogy with Definitions 2.2 and 2.3 . Both the coefficients of $L_{E, E^{\prime}}^{+}$and the scalar products $(u, v)_{E}$ and $\left(u^{\prime}, v^{\prime}\right)_{E^{\prime}}$ involve the matrix-valued functions $E(x)$ and $E^{\prime}(x)$. If $E(x)$ and $E^{\prime}(x)$ and their first derivatives are continuous and if (1.5) holds for both, then it can again be shows that the operators $L_{s}$ and $I_{w}$ coincide and define the same linear operator $L\left(=L_{s}=L_{w}\right.$ ) as in the case where $E=I$ and $E^{\prime}=I^{\prime}$. This result is needed below only for differential operators $\Lambda$ of the form (1.13). Therefore, this section is concluded with a discussion of this ease.

Consider the differential operators

$$
\Lambda=-i E(x)^{-1} \sum_{j=1}^{n} A_{j} D_{j}
$$

and

$$
\Lambda_{0}=-i E_{0}^{-1} \sum_{j=1}^{n} A_{j} D_{j}
$$

where, as in $\S 1, E(x), E_{0}, A_{1}, \ldots, A_{n}$ are $n \times m$ Hermitian matrices, $E_{0}$, $A_{1}, \ldots, A_{n}$ are constant, $E(x)$ and $E_{0}$ are positive definite and $E(x)$ satisfies (1.5). In the paper [22] the notation

$$
H=L_{2, m}(E), \quad H_{0}=L_{2, m}\left(E_{0}\right)
$$

was used and the differential operators $A$ and $\Lambda_{0}$ were used to define selfadjoint operators $\Lambda$ and $\Lambda_{0}$ on $H$ and $H_{0}$ respectively. T.e operator $\Lambda_{0}: H_{0} \rightarrow H_{0}$ was defined in [22] by means of the Fourien transform (1.19) and the symbol

$$
\Lambda 0(p)=E_{0}^{-1} \sum_{j=1}^{n} A_{j} p_{i}
$$

as follows.

Definitrox 2.4. $-\Lambda: H_{0} \rightarrow H_{0}$ is the linear operator defined by the conditions that

$$
D\left(\Lambda_{0}\right)=H_{0} \cap ; u: \Lambda_{0}, \widehat{p} \widetilde{u}(p) \in H_{0} !
$$

and $\Lambda_{0} u=v$ if and only if $\widehat{v}(p)=\Lambda_{0}(p) \bar{u}(p)$. 
J. R. Schulenberger-C. H. Wrlcox: Coerciveness inequalities, etc.

Note that multiplication by $E(x)$ defines an operator $E$ on $H$ which is bounded by (1.5). Moreover, multiplication by $E(x)^{-1}$ defines the inverse operator $E^{-1}$ which is also bounded because $E(x)^{-1}$ satisfies (1.5) when $E(x)$ does. The same remarks apply to $E_{0}$ and $E_{0}^{-1}$. With this notation the operator $\Lambda$ was defined in [22] as follows.

Definimion 2.5. $-\Lambda: H \rightarrow H$ is the linear operator defined by

$$
D(\Lambda)=D\left(\Lambda_{0}\right)
$$

and

$$
\Lambda=E^{-1} E_{0} \Lambda_{0} .
$$

Let $J$ denote the identification map from $H_{0}$ onto $H$ :

$$
J: H_{0} \rightarrow H, \quad J u=u \text { for all } u \in H_{0} .
$$

Then $J$ and $J^{-1}$ are bounded operators because the norms in $H_{0}$ and $H$ are equivalent. Since $\Lambda$ and $E$ are regarded as operators on $H$ and $\Lambda_{0}$ and $E_{0}$ as operators on $H_{0}$, it would be correct to define $\Lambda$ in terms of $\Lambda_{0}$ by

$$
D(\Lambda)=J D\left(\Lambda_{0}\right)
$$

and

$$
\Lambda=E^{-1} J E_{0} \Lambda_{0} J^{-1}
$$

rather than (2.31) and (2.32). In many cases the operator $J$ can be omitted, as in Definition 2.5, without confusion. However, it is sometimes important to recognize that $J^{*}$, the adjoint of $J$, is not the identification map but

$$
J^{*}=E_{0}^{-1} J^{-1} E \text {. }
$$

This fact is used below in 85 .

Definitions 2.4 and 2.5 were given in [22] where it was shown that the operators $\Lambda_{0}$ and $\Lambda$ so defined are selfadjoint on $H_{0}$ and $H$ respectively. These definitions differ from Definitions 2.2 and 2.3 of the strong extension $\Lambda_{s}$ and the weak extension $\Lambda_{w}$. Nevertheless, these operators all coincide if $E(x) \in C^{1}\left(R^{n}\right)$ and satisfies $(1.5)$.

This is proved below, following two preliminary lemmas.

Lemma 2.4. - Let $\Lambda_{0}$ be defined by Definition 2.4. Then $u \in D\left(\Lambda_{0}\right)$ and $\Lambda_{0} u=v$ if and only if

$$
\left(u, \Lambda_{0} \varphi\right)_{E_{0}}=(v, \varphi)_{E_{0}} \text { for all } \varphi \in C_{0}^{1}\left(R^{n} ; C^{m}\right) .
$$


The proof of this is immediate from Definition 2.4 and the PLancherel theory and is omitted.

Lemma 2.5. - Let $\Lambda$ be defined by Definition 2.5. Then $u \in D(\Lambda)$ and $\Lambda u=v$ and only if

$$
(u, \Lambda \varphi)_{E}=(v, \varphi)_{E} \text { for all } \varphi \in C_{0}^{1}\left(R^{n} ; C^{m}\right)
$$

Proof. - Note that

$$
(u, \Lambda \varphi)_{E}=\left(u, \Lambda_{0} \varphi\right)_{E_{0}}
$$

for all $u \in H$ and $\varphi \in C_{0}^{1}\left(R^{n} ; C^{m}\right)$. Moreover,

$$
(v, \varphi)_{E}=\left(E_{0}^{-1} E v, \varphi\right)_{E_{0}} \text { and }
$$

$$
(w, \varphi)_{E_{0}}=\left(E^{-1} E_{0} w, \varphi\right)_{E}
$$

for all $u, v$ and $\varphi$ in $H$. Now assume that $u \in D(\Lambda)=D\left(\Lambda_{0}\right)$ and $\Lambda u=$ $=E^{-1} E_{0} \Lambda_{0} u=v$. Then, by $(2.36)$ and $(2.37)$

$$
(u, \Lambda \varphi)_{E}=\left(u, \Lambda_{0} \varphi\right)_{E_{0}}=\left(\Lambda_{0} u, \varphi\right)_{E_{0}}=\left(E^{-1} E_{0} \Lambda_{0} u, \varphi\right)_{E}=(v, \varphi)
$$

for all $\varphi \in C_{0}^{1}\left(R^{n} ; C^{m}\right)$. Conversely. if (2.35) holds then (2.36) and (237) imply

$$
\left(u, \Lambda_{0} \varphi\right)_{E_{0}}=(u, \Delta \varphi)_{E}=\left(v, \varphi_{, E}^{\prime}=\left(E_{0}^{-1} E v, \varphi\right)_{E_{0}}\right.
$$

for all $\varphi \in C_{0}^{1}\left(R^{n} ; C^{m}\right)$. Thus Lemma 2.4 implies that $u \in D\left(\Lambda_{0}\right)=D(\Lambda)$ and $\Lambda_{0} u=E_{0}^{-1} E v$, whence $\Lambda u=E^{-1} E_{0} \Lambda_{0} u=v$, which completes the proof.

Definition 2.3 of $\Lambda_{w}$ makes ase of operator $\Lambda^{+}$, the formal adjoint of $\Lambda$ as an operator on $L_{2, m}(I)$. By $(2.7)$ and $(2.23)$

$$
\Lambda^{\dagger} \varphi(x)=-i \sum_{j=1}^{n} A_{j} D_{j} ; E(x)^{-1} \varphi(x) !
$$

since $A_{j}$ is constant and $A_{j}$ and $E(x)$ are Hermitrax. Thus $u \in D\left(\Lambda_{w}\right)$ and $\Lambda_{w} u=v$ if and only if

$$
\left(u, \Lambda^{+} \varphi\right)=(v, \varphi) \text { for all } \varphi \in C_{0}^{l}\left(R^{n} ; C^{m}\right)
$$

This implies

Theonem 2.6. - Assume that $E(x) \in C^{1}\left(R^{n}\right)$ and satisfies (1.5) Then $\Lambda_{v}=\Lambda$. 
Proof. - Note that $E$ defines a mapping of $C_{0}^{\mathrm{l}}\left(R^{n} ; C^{m}\right)$ onto itself with inverse $E^{-1}$; i.e. $\varphi=E \psi \in C_{0}^{1}\left(R^{n} ; C^{m}\right)$ if and only if $\psi=E^{-1} \varphi \in C_{0}^{1}\left(R^{n} ; C^{m}\right)$. Thns if $u \in D\left(\Lambda_{w}\right)$ and $\Lambda_{w} u=v$ then

$$
\begin{gathered}
(v, \psi)_{E}=\left(v, E^{-1} \varphi\right)_{E}=(v, \varphi)=\left(u, \Lambda^{\dagger} \varphi\right)= \\
\int_{R^{n}} u(x)^{*}\left(-i \sum_{j=1}^{n} A_{j} D_{j}\left\{E^{-1}(x) \varphi(x) !\right) d x=\int_{R^{n}} u(x)^{*}\left(-i \sum_{j=1}^{n} A_{j} D_{j} \psi(x)\right) d x=\right.
\end{gathered}
$$

$$
\int_{R^{n}} u(x)^{*} E(x)\left(-i E(x)^{-1} \sum_{j=1}^{n} A_{j} D_{j} \psi(x)\right) d x=(u, \Lambda \psi)_{E}
$$

for all $\psi \in C_{0}^{1}\left(R^{n} ; C^{m}\right)$. Thus $u \in D(\Lambda)$ and $\Lambda u=v$; i.e. $\Lambda_{w} \subset \Lambda$.

Conversely, if $u \in D(\Lambda)$ and $\Lambda u=v$ then as above

$$
(v, \varphi)=(v, \psi)_{E}=(u, \Lambda \psi)_{E}=\left(u, \Lambda^{\dagger} \varphi\right)
$$

for all $\varphi \in C_{0}^{\mathrm{l}}\left(R^{n} ; C^{m}\right)$. Thus $u \in D\left(\Lambda_{w}\right)$ and $\Lambda_{w} u=v$, i.e. $\Lambda \subset \Lambda_{t v}$. Thus $\Lambda=\Lambda_{w}$.

Corollari $2.7 \cdot-\Lambda_{0}=\Lambda_{0, w}$.

Proof. - This follows immediately from Theorem 2.6 with $E(x)=E_{0}$.

\section{§ 3. - Coerciveness Inequalities for Elliptic Operators.}

In this section coereiveness inequalities are derived for a class of elliptic operators on $R^{n}$. The results given here are either known or very similar to known results. The purpose of the section is to establish the results and techniques for elliptic systems that are used in the study of nonelliptic systems in the following sections.

The derivation of coerciveness inequalities for elliptic systems is based on a reformulation of the ellipticity condition contained in the following two lemmas.

Lemma 3.1. - The differential operator $L$ is elliptic at $x_{0} \in R^{n}$ if and only if it has the property that for all $p \in R^{n}-\{0\}$ and $\xi \in C^{m}$,

$$
L\left(p, x_{0}\right) \xi=0 \text { implies that } \xi=0 .
$$

Condition (3.1) is clearly equivalent to condition (1.10) of Definition 1.3. 
Lemma 3.2. - The differential operator $L$ is elliptic at $x_{0} \in R^{n}$ if and only if there exists a constant $\mu\left(x_{0}\right)>0$ such that

$$
\left|L\left(p, x_{0}\right) u\right|^{2} \geq \mu\left(x_{0}\right)^{2}|p|^{2}|u|^{2} \text { for all } p \in R^{n} \text { and } u \in C^{m}
$$

(The norm $|\cdot|$ ean be any matrix norm).

Proof. - Write $p=|p| \eta$ and $u=|u| \tilde{u}$ where $\eta$ and $\tilde{u}$ are unit vectors. Then $\left|L\left(\eta, x_{0}\right) \tilde{u}\right|$ is continuous in $\eta$ and $\tilde{u}$, and hence it has a lower bound $\mu\left(x_{0}\right) \geq 0$ which is achieved for some $\eta_{0}$ and $\tilde{u}_{0}$, since the unit spheres are compact. If $\mu\left(x_{0}\right)=0$ then $l\left(\eta_{0}, x_{0}, \tilde{u}_{0}=0\right.$ and $L$ is not elliptic at $x_{0}$ by Lemma 3.1. Thus if $L$ is elliptic at $x_{0}$ then $\left|L\left(\eta, x_{0}\right) \tilde{u}\right|^{2} \geq \mu\left(x_{0}\right)>0$. This im. plies $(3.2)$, since $L\left(p, x_{0}\right) u$ is homogencons of degree one in $p$ and $u$. Conversely, if (3.2) holds with $\mu\left(x_{0}\right)>0$ then $L\left(p, x_{0}\right) u=0$ implies $u=0$ for all $p \in R^{n}-\{0\}$. Hence $L$ is elliptic at $x_{0}$ by Lemma 3.1.

Definimion 3.1. - The differential operator $L$ is uniformly elliptic in a region $\Omega \subset R^{n}$ if and only if there exists a constant $\mu>0$ such that

$$
|L(p, x) u|^{2} \geq \mu^{2}|p|^{2}|u|^{2} \text { for all } p \in R^{n}, u \in C^{m} \text { and } x \in \Omega \text {. }
$$

A sufficient condition for uniform ellipticity in $R^{n}$ is given by

Lemma 3.3. - Let $L$ be a differential operator which satisfies (2.1), (2.2). Assume that $L$ is elliptic in $R^{n}$ and that

$$
\lim _{|x| \rightarrow \infty} L_{i j}(x)=L_{j}^{\infty}, j=1, \ldots, n,
$$

uniformly in $x /|x|$ where

$$
l^{\infty}=-i \sum_{j=1}^{n} l_{j}^{\infty} D_{j}
$$

is an elliptic operator. Then $L$ is uniformly elliptic in $R^{n}$.

Proof. - Let $r$ be any positive number. Then $|L(\eta, x) \tilde{u}|$ is continuous and never zero on the compact set $\left\{(r, x, \tilde{u}):|\eta|=1,\left|x^{\prime} \leq r,\right| \tilde{u} \mid=1\right\} \cdot$ Hence there is a positive constant $\mu(r)$ such that

$$
|L(\eta, x) \tilde{u}| \geq \mu(r) \text { for } \quad|\eta|=1,|x| \leq r,|\tilde{u}|=1
$$

Next

$$
L\left(\eta_{1}, x\right) \tilde{u}-L^{\infty}(\eta) \tilde{u}=\sum_{j=1}^{n}\left[L_{j}(x)-L_{l}^{\infty}\right] \eta_{j} \tilde{u}
$$


whence

$$
\left|h(\eta, x) \tilde{u}-L^{\infty}(\eta) \tilde{u}\right| \leq \sum_{j=1}^{n}\left|\left[L_{j}(x)-l_{j j}^{\infty}\right] \tilde{u}\right| \leq \sum_{j=1}^{n}\left|L_{j j}(x)-L_{j}^{\infty}\right|
$$

for all $(\eta, x, \tilde{u})$ with $|\eta|=1, x \in R^{n}$ and $|\tilde{u}|=1$. Thus (3.4) implies that

$$
\lim _{|x| \rightarrow \infty} L(\eta, x) \tilde{u}=L^{\infty}(\eta) \tilde{u} \text { uniformly in } x /|x|, \eta \text { and } \tilde{u} \text {. }
$$

Now, since $L^{\infty}$ is elliptic there is a $\mu_{1}>0$ such that

$$
\left|L^{\infty}(\eta) \tilde{u}\right| \geq \mu_{1} \quad \text { for } \quad|\eta|=1,|\tilde{u}|=1
$$

Thus (3.9) implies that there is an $r_{0}>0$ such that

$$
|| L(\eta, x) \tilde{u}|-| L^{\infty}(\eta) \tilde{u}|| \leq 2^{-1} \mu_{1}
$$

$$
\text { for }|\eta|=1,|x| \geq r_{0},|\tilde{u}|=1
$$

Hence

$$
|\operatorname{li}\rangle, x) \tilde{u}|=| L^{\infty}(\eta) \tilde{u} \mid+\left(|L(\eta, x) \tilde{u}|-\left|L^{\infty}(\eta) \tilde{u}\right|\right) \geq
$$

$$
\mu_{1}-2^{-1} \mu_{1}=2^{-1} \mu_{1}
$$

for $|\eta|=1,|x| \geq r_{0}$ and $; \tilde{u} \mid=1$. Combining (3.6) with $r=r_{0}$ and $\mu\left(r_{0}\right)=\mu_{0}$ and (3.12) gives

$$
|L(\eta, x) \tilde{u}| \geq \mu=\min \left(\mu_{0}, 2^{-1} \mu_{1}\right)>0
$$

for $|\eta|=1, x \in R^{\mu}$ and $|\tilde{u}|=1$. Finally, since $L(p, x) u$ is homogeneous of degree one in $(p, u)(3.13)$ implies that (3.3) holds for all $x \in R^{n}, p \in R^{n}$ and $u \in C^{m}$.

The coerciveness prcperty for an operator $L: L_{2, m} \rightarrow L_{2, u^{\prime}}$ can be reformulated in terms of the Sobouev space

$$
L_{2, m}^{1}=L_{2, m} \cap: \boldsymbol{u}: D_{j} u \in L_{2, m} \quad \text { for } \quad j=1,2, \ldots, \boldsymbol{u}
$$

$L_{2, m}^{1}$ is a HrLbert space whith inner produet

$$
(u, v)_{1}=\int_{R^{n}}\left(u^{*} v+\sum_{j=1}^{n} D_{j} u^{*} D_{j} u\right) d x
$$

and norm $\|u\|_{1}=(u, u)_{1}^{1 / 2}$. 
Lemas 3.4. - The operator $L_{1}: L_{2, m} \rightarrow L_{2, m^{\prime}}$ is coeroive if and only if $D(L)=L_{2, n}^{1}$ and there exists a constant $K>0$ such that for all $u \in D(L)$

$$
\|u\|_{1}^{2} \leq K^{2}\left(\|L u\|^{2}+\|u\|^{2}\right)
$$

Condition (3.16) is obviously equivalent to (1.8), with a different constant $K$, since

$$
\|u\|_{1}^{2}=\|u\|^{2}+\sum_{j=1}^{n}\left\|D_{j} u\right\|^{2} .
$$

The principal result of this section is

Theorem 3.5. - Let the differential operator $L$ have the properties

(1) $L$ is uniformly elliptic in $R^{n}$,

(2) The coefficients $L_{0}(x), L_{1}(x), \ldots, L_{n}(x)$ satisfy conditions (2.1) and (2.2).

Then the closed linear operator $L: L_{2, m} \rightarrow L_{2, m^{\prime}}$ associated with $L$ is coercive.

Proof. - Since $L=L_{s}$ is the closure of the minimal operator $\tilde{L}$ it suffices to prove that there exists a $K>0$ such that $(3.16)$ holds for all $u \in C_{0}^{1}\left(R^{n} ; C^{m}\right)$. Indeed, if this true and $u \in D(L)$ then there exists a sequence $\left\{\boldsymbol{u}_{k}\right\} \subset C_{0}^{1}\left(R^{n} ; C^{m}\right)$ such that $u_{k} \rightarrow u$ in $L_{2, m}$ and $L u_{k} \rightarrow L u$ in $L_{2, m^{\prime}}$. (3.16) holds for each $u_{k}$ and for the differences $u_{k}-u_{l}$. It follows that $; u_{k}$ ! is a $\mathrm{C}_{A U C H Y}$ sequence in $L_{2, m}^{1}$ and hence $u \in L_{2, m}^{1}$. Thus $D(L) \subset L_{2, m}^{1}$. Finally, writing (3.16) for $u_{k}$ and making $k \rightarrow \infty$ gives (3.16) for $u \in D, L$ ).

The proof of $(3.16)$ for $\left.u \in C_{0}^{1} R^{n} ; C^{m}\right)$ is exactly analogous to a proof of GaRDING's inequality due to $\mathrm{S}$. AGMoN [3]. The main steps are given here to show how the method applies to elliptic systems with $w^{\prime}$ equations and $m$ unknows $\left(m^{\prime} \geq m\right)$.

Let

$$
L_{p}=-i \sum_{j=1}^{n} L_{j}(x) D_{j}
$$

denote the principal part of $L$. Then if $u \in C_{0}^{\mathrm{I}}\left(R^{n} ; C^{m}\right)$,

$$
\left\|L_{p} u\right\|^{2}=\int_{R^{n}}\left(L_{p} u\right)^{*} L_{p} u d x=\int_{R^{n}}\left(\sum_{j=\mathrm{l}}^{n} L_{j} D_{j} u\right)^{*} \sum_{k=1}^{n} L_{k} D_{k} u d x=
$$

$$
\int_{R^{n}}\left(\sum_{j=1}^{n} \sum_{k=1}^{n} D_{j} u^{*} L_{j}^{*} L_{k} D_{k} u\right) d x=\int_{R^{n}}\left(\sum_{j=1}^{n} \sum_{k=1}^{n} D_{j} u^{*} T_{j k}(x) D_{k} u\right) d x
$$

where $T_{j k}(x)=L_{j}^{*}(x) L_{k}(x)$ is an $m \times m$ matrix. 
J. R. Schulenberger - C. H. Wilcox: Coerciveness inequalities, etc. 253

Lemma 3.6. - If $L_{p}$ has constant coefficients $L_{j}(j=1, \ldots, n)$ then for all $u \in C_{0}^{\mathrm{l}}\left(R^{n} ; C^{m}\right)$,

$$
\left\|L_{p} u\right\|^{2} \geq \mu^{2} \sum_{j=1}^{n}\left\|D_{j} u\right\|^{2}
$$

where $\mu$ is the ellipticity constant from (3.3).

Proom. - The proof is the same as the proof of (1.55) for the equations of elasticity. By the Planoherel theory

$$
\begin{gathered}
\left\|L_{p} u\right\|^{2}=\int_{R^{n}} \tilde{u}(p)^{*} \sum_{j=1}^{n} \sum_{k=1}^{n} L_{j}^{*} p_{j} L_{k} p_{k} \tilde{u}(p) d p= \\
\int_{R^{n}}|L(p) \tilde{u}(p)|^{2} d p \geq \mu^{2} \int_{R^{n}}|p|^{2}|\tilde{u}(p)|^{2} d p=\mu^{2} \sum_{j=1}^{n}\left\|D_{j} u\right\|^{2} .
\end{gathered}
$$

Lemma 3.7. - Under the hypotheses of Theorem 3.5, there exists a constant $\rho>0$ such that

$$
\left\|L_{p} u\right\|^{2} \geq 2^{-1} \mu^{2} \quad \sum_{j=1}^{n}\left\|D_{j} u\right\|^{2}
$$

for all $\left.u \in C_{0}^{1} R^{n} ; C^{n}\right)$ such that supp $u$, the support of $u$, has diameter less than $\rho . \rho$ depends only on $n$ and the moduli of continuity of $L_{1}(x), \ldots, L_{n}(x)$.

Proof. - It is easy to show that (2.1) implies that $L_{1}(x), \ldots, L_{n}^{\prime}(x)$ are uniformly continuous on $R^{n}$. Hence each of the matrices $T_{j k}(x)=L_{j}^{*}(x) L_{p}(x)$ is uniformly continuous on $R^{n}$. Thus, gives any $\varepsilon>0$ there exists a $\rho>0$ such that

$$
\left.\mid T_{j k^{\prime}} x\right)-T_{j k^{\prime}}\left(x_{0}\right) \mid \leq \varepsilon \quad \text { f } \mathrm{r}^{\mathrm{r}} \quad 1 \leq j, k \leq n,
$$

provided that $\left|x-x_{0}\right| \leq \rho . \rho$ depends on $\varepsilon$ and the moduli of continuity of the $T_{j k}(x)$. Let $u \in O_{0}^{I}\left(R^{n} ; C^{m}\right)$ and let supp $u$ have diameter less than $\rho$. Let $x_{0} \in \operatorname{supp} u$ and define

$$
L_{p, 0}=-i \sum_{j=1}^{n} L_{j}\left(\boldsymbol{x}_{0}\right) D_{j}
$$

Then

$$
\begin{gathered}
\left\|L_{p} u\right\|^{2}-\left\|L_{p, 0} u\right\|^{2}= \\
\int_{\text {snpp } z} \sum_{j=1}^{n} \sum_{k=1}^{n}\left(D_{j} u\right)^{*}\left[T_{j k}(x)-T_{j k}\left(x_{0}\right)\right] D_{k i}^{\prime K} u d x
\end{gathered}
$$


254 J. R. Schulenberger - C. H. Wilcox: Coerciveness inequalities, etc.

and hence

$$
\begin{aligned}
& \left|\left\|L_{p} u\right\|^{2}-\left\|L_{p, 0} u\right\|^{2}\right| \leq \\
& \int_{\text {supp } u} \sum_{j=1}^{n} \sum_{k=1}^{n}\left|T_{j k}(\boldsymbol{x})-T_{j k}\left(x_{0}\right)\right| \cdot\left|D_{j} u\right| \cdot\left|D_{k} u\right| d x \leq
\end{aligned}
$$

$$
\begin{aligned}
\varepsilon \sum_{j=1}^{n} \sum_{k=1}^{n} & \int_{R^{n}}\left|D_{j} u\right| \cdot\left|D_{k} u\right| d x \leq \varepsilon / 2 \sum_{j=1}^{n} \sum_{k=1}^{n} \int_{R^{n}}\left(\left|D_{j} u\right|^{2}+\left|D_{k} u\right|^{2}\right) d x= \\
& \boldsymbol{n} \varepsilon \sum_{j=1}^{n}\left\|D_{j} \boldsymbol{u}\right\|^{2} .
\end{aligned}
$$

Inequality (3.26) implies

$$
\left\|L_{p} u\right\|^{2} \geq\left\|L_{p, 0} u\right\|^{2}-n \varepsilon \sum_{j=1}^{n}\left\|D_{j} u\right\|^{2} .
$$

Hence, by Lemma 3.6,

$$
\left\|L_{p} u\right\|^{2} \geq\left(\mu^{2}-n \varepsilon\right) \sum_{j=1}^{n}\left\|D_{j} u\right\|^{2}=2^{-1} \mu^{2} \sum_{j=1}^{n}\left\|D_{j} u\right\|^{2}
$$

provided $n \varepsilon=(1 / 2) \mu^{2}$. This choice of $\varepsilon$ determines $\rho$.

Lemma 3.8. - Under the hypothesis of Theorem 3.5, there exists a constant $K_{1}$ such that

$$
\|L u\|^{2} \geq\left\|L_{p} u\right\|^{2}-K_{1}\|u\| \cdot\|u\|_{1},
$$

for all $u \in C_{0}^{1}\left(R^{n} ; C^{m}\right)$. $K_{1}$ depends only on $n$ and the bounds for the coefficients $\left.L_{0}{ }^{\prime} x\right), L_{1}(x), \ldots, L_{n}(x)$.

Proof. $-L=L_{p}+L_{0}$. Hence

$$
\begin{aligned}
\|L u\|^{2} & =\int_{R^{n}}\left(L_{p} u+L_{0} u\right)^{*}\left(L_{p} u+L_{0} u\right) d x=\int_{R^{n}}\left(\left(L_{p} u\right)^{*}\left(L_{p} u\right)+\right. \\
& \left.+\left(L_{p} u\right)^{*}\left(L_{0} u\right)+\left(L_{0} u\right)^{*}\left(L_{p} u\right)+L_{0} u\right)^{*}\left(L_{0} u\right) ! d x \\
& =\left\|L_{p} u\right\|^{2}+R_{1}(u)
\end{aligned}
$$

where

$$
R_{\mathrm{I}}(u)=\int_{R^{n}}\left\{i \sum_{j=1}^{n} D_{j} u^{*} L_{p}^{*} L_{0} u-i \sum_{j=1}^{n} u^{*} L_{0}^{*} L^{j} D_{j} u+u^{*} L_{0}^{*} L_{0} u \vdots d x\right.
$$


Now

$$
\left|\int_{R^{\prime 2}} D_{j} u^{*} L_{P}^{*} L_{0} u d x\right| \leq \int_{R^{\prime 2}}\left|D_{j} u\right| \cdot\left|u_{1}^{\prime} \cdot\right| L_{p}^{*} L_{0} \mid d x \leq K_{2}\|u\| \cdot\|u\|_{1}
$$

where $K_{2}$ depends on the bounds for the $L_{j}$. The second term in (3.31) can be estimated in the same way, and

$$
\left|\int_{R^{n}} u^{*} L_{0}^{*} L_{0} u d x\right| \leq K_{3}\|u\|^{2}
$$

Thus

$$
\left|R_{1}(u)\right| \leq \sum_{j=1}^{n} 2 K_{2}\|u\| \cdot\|u\|_{1}+K_{3}\|u\|^{2} \leq K_{1}\|u\| \cdot\|u\|_{1}
$$

where $K_{1}=2 n K_{2}+K_{3} .(3.30)$ and (3.34) imply (3.29).

Proof of Theorem 3.5, concluded. - The proof makes use of Lemmas 3.6, 3.7 and 3.8 and a special partition of unit $\left\{\xi_{x}(x)\right\}$, due to S. AGMon [3], with the following properties: $\alpha=\left(\alpha_{I}, \ldots, \alpha_{n}\right)$ is an $n$-tuple of integers, $|\alpha|=\alpha_{1}+$ $+\ldots+\alpha_{n}$, and

$$
\sum_{\mid x_{i}=0}^{\infty} \zeta_{x}(x)^{2}=1 \text { for all } x \in R^{n}
$$

The functions $\zeta_{\alpha}$ are defined by

$$
\zeta_{x}=\zeta_{\alpha}^{\prime}\left[\sum_{\beta} \zeta_{\beta}^{\prime 2}\right]^{-1 / 2}, \zeta_{\beta}^{\prime}(x)=\zeta^{\prime}(x-d \beta)
$$

where

$$
\zeta^{\prime}(x) \in C_{0}^{\infty}\left(R^{n}\right), 0 \leq \zeta(x) \leq 1, \text { and } \operatorname{supp} \zeta \subset Q_{0}
$$

where

$$
\left.Q_{a}=\left\{x: d \alpha_{k}-1\right)<x_{k}<d\left(\alpha_{k}+1\right), k=1,2, \ldots, n\right\}
$$

is the cube with center at $d \alpha$ and side $2 d$. Note that supp $\zeta_{\alpha} \subset Q_{\alpha}$.

To prove the coereiveness inequality (3.16) for arbitrary $u \in C_{0}^{1}\left(R^{n} ; C^{m}\right)$, choose $d$ so that the diameter of $Q_{x}$ is less than the number $\rho$ of Lemma 3.7; i.e. $2 d \sqrt{n}<\rho$, so that Lemma 3.7 is applicable to the function $\zeta_{\alpha} u$. Then, by (3.19) and (3.35) 


$$
\begin{aligned}
\left\|L_{p} u\right\|^{2} & =\int_{R^{n}} \sum_{|\alpha|=0}^{\infty} \sum_{j=1}^{n} \sum_{k=1}^{n} \zeta_{x}(x)^{2} D_{j} u(x)^{*} T_{j k}(x) D_{k} u(x) d x \\
& =\int_{R^{n}} \sum_{|\alpha|=0}^{\infty} \sum_{j=1}^{n} \sum_{k=1}^{n} D_{j}\left(\zeta_{\alpha} u\right)^{*} T_{j k}(x) D_{k}\left(\zeta_{\alpha} u\right) d x+R_{2}(u) \\
& =\sum_{|\alpha|=0}^{\infty}\left\|L_{p}\left(\zeta_{x} u\right)\right\|^{2}+R_{2}(u)
\end{aligned}
$$

where

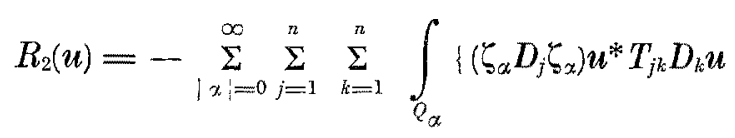

$$
\left.+\left(\zeta_{\alpha} D_{k} \zeta_{x}\right) D_{j} u^{*} T_{j k} u+\left(D_{j} \zeta_{\alpha} D_{k} \zeta_{x}\right) u^{*} T_{j k} u\right) d x
$$

Notice that all the sums in (3.39) and (3.40) are finite because supp $u$ meets only a finite number of the cubes $Q_{\alpha}$. Let

$$
\left|D_{j} \zeta_{a}\right| \leq K_{4} \text { for } j=1,2, \ldots, n \text { and all } \alpha
$$

$\left(K_{4}\right.$ depends only on $d$; i.e. $\rho$ and $\left.n\right)$. Then

$$
\left|R_{2}(u)\right| \leq \sum_{|x|=0}^{\infty} \sum_{j=1}^{n} \sum_{k=1}^{n} \int_{Q_{\alpha}}\left\{K_{4}\left|u^{*} T_{j k} D_{k} u\right|+K_{4}\left|D_{j} u^{*} T_{j k} u\right|+\right.
$$

$$
K_{4}^{2}\left|u^{*} T_{j k} u\right| ! d x
$$

Note that, apart from a null set, every $x \in R^{n}$ is in precisely $2^{n}$ of the cubes $Q_{\alpha}$. Thus

$$
\begin{gathered}
\left|R_{2}(u)\right| \leq 2^{n} \sum_{j=1}^{n} \sum_{k=1}^{n} \int_{R^{n}}\left\{K_{4}\left|u^{*} T_{j k} D_{k} u\right|+K_{4}\left|D_{j} u^{*} T_{j k} u\right|+\right. \\
K_{4}^{2}\left|u^{*} T_{j k} u\right|^{\prime} d x \leq 2^{n} \sum_{j=1}^{n} \sum_{k=1}^{n} \int_{R^{n}} \mid K_{4} K_{5}\|u\| \cdot\left\|D_{k} u\right\|+ \\
K_{4} K_{5}\left\|D_{j} u\right\| \cdot\|u\|+K_{4}^{2} K_{5}\|u\| \|^{2} ! d x
\end{gathered}
$$

where $K_{5}$ is a bound for $\left\|T_{j k}\right\|$. Thus, by Sorwarz's inequality 
J. R. Schulenberger - C. H. Wilcox: Coerciveness inequalities, etc.

$$
\left|R_{2}(u)\right| \leq 2^{n}\left(K_{4} K_{5} n\|u\| \sum_{j=1}^{n}\left\|D_{k} u\right\|+K_{4} K_{5} n\|u\| \sum_{k=1}^{n}\left\|D_{j} u\right\|+\right.
$$

$$
\left.+K_{4}^{2} K_{5} n^{2}\|u\|^{2}\right) \leq K_{6}\|u\| \cdot\|u\|_{I}
$$

where $K_{6}$ depends only on $n, \rho$ and the bounds for the coefficients $L_{f}(x)$.

Thus

$$
\left\|L_{p} u\right\|^{2} \geq \sum_{|x|=0}^{\infty}\left\|L_{p}\left(\zeta_{\alpha} u\right)\right\|^{2}-K_{6}\|u\| \cdot\|u\|_{1}
$$

Combining this with Lemma 3.8 gives

$$
\|L u\|^{2} \geq \sum_{|\alpha|=0}^{\infty}\left\|L_{p}\left(\zeta_{\alpha} u\right)\right\|^{2}-K_{7}\|u\| \cdot\|u\|_{1}
$$

where $K_{7}=K_{1}+K_{6}$. Now, application of Lemma 3.7 to the function $\zeta_{\alpha} u$ gives

$$
\left\|L_{p}\left(\zeta_{x} u\right)\right\|^{2} \geq(1 / 2) \mu^{2} \quad \sum_{j=1}^{n}\left\|D_{j}\left(\zeta_{x} u\right)\right\|^{2}
$$

Moreover,

whence

$$
\begin{aligned}
& \left\|D_{j}\left(\zeta_{\alpha} u\right)\right\|^{2}=\int_{R^{n}}\left\|\zeta_{\alpha} D_{j} u+\left(D_{j} \zeta_{x}\right) u\right\|^{2} d x \\
& =\int_{R^{n}} S_{2}^{2} D_{j} u^{*} D_{j} u+\zeta_{\alpha}\left(D_{j} \zeta_{x}\right)\left(D_{j} u^{*}\right) u+\left(D_{j} \zeta_{\alpha}\right) \zeta_{\alpha} u^{*} D_{j} u+ \\
& \left.+\left(D_{J} \zeta_{x}\right)^{2} u^{*} u\right\} d x
\end{aligned}
$$

$$
\left.\sum_{|\alpha|=0}^{\infty} \sum_{j=1}^{n}\left\|D_{j}\left(\zeta_{x} u\right)\right\|^{2}=\sum_{j=1}^{n}\left\|D_{j} u\right\|^{2}+R_{3} u\right)
$$

where

$$
R_{3}(u)=\sum_{|\alpha|=0}^{\infty} \sum_{j=1}^{n} \int_{Q_{\alpha}}\left\{\zeta_{\alpha} D_{j} \zeta_{\alpha}\left(D_{j} u^{*}\right) u+\left(D_{j} \zeta_{\alpha}\right) \zeta_{\alpha} u^{*} D_{j} u+\right.
$$

$$
\left.+\left(D_{j} \zeta_{\alpha}\right)^{2} u^{*} u\right\} d x
$$

This is similar in structure to $R_{2}(u)$ and can be estimated in the same way:

$$
\left|R_{3}(u)\right| \leq K_{8}^{r}\|u\| \cdot\|u\|_{1} .
$$


Combining (3.46), (3.47), (3.49) and (3.51) gives

$$
\begin{aligned}
\|L u\|^{2} & \geq 2^{-1} \mu^{2}\left(\sum_{j=1}^{n}\left\|D_{j} u\right\|^{2}-K_{8}\|u\|\|u\|_{1}\right)-K_{7}\|u\| \cdot\|u\|_{1} \\
& =2^{-1} \mu^{2}\left(\|u\|_{1}^{2}-\|u\|^{2}\right)-\left(K_{7}+2^{-1} \mu^{2} K_{8}\right)\|u\| \cdot\|u\|_{1} \\
& =2^{-1} \mu^{2}\|u\|_{1}^{2}-K_{9}\|u\| \cdot\|u\|_{1}-2^{-1} \mu^{2}\|u\|^{2}
\end{aligned}
$$

where $K_{9}=K_{7}+(1 / 2) \mu^{2} K_{8}$. Now, the inequality $2 a b \leq \delta a^{2}+\delta^{-1} b^{2}$, applied to the term $\|u\| \cdot\|u\|_{i}$ gives

$$
\|L u\|^{2} \geq\left(2^{-1} \mu^{2}-2^{-1} K_{9} \delta\right)\|u\|_{1}^{2}-\left(2^{-1} \mu^{2}+2^{-1} K_{9} \delta-1\right)\|u\|^{2} .
$$

Choose $\delta$ so that $2^{-1} K_{9} \delta=4^{-1} \mu^{2}$. Then

$$
\|L u\|^{2} \geq(1 / 4) \mu^{2}\|u\|_{1}^{2}-K_{10}\|u\|^{2} .
$$

This is equivalent to (3.16), which completes the proof of Theorem 3.5.

Theorem 3.5 states that a differential operator $L$ is coercive if it is uniformly elliptic and its coefficients satisfy hypotheses (2.1) and (2.2). The converse is also true under the same hypotheses on the coefficients. This assertion is implied by

Theorem 3.9. - Let the coefficients $L_{0}(x), L_{1}(x), \ldots, L_{n}(x)$ satisfy conditions (2.1) and (2.2), and let the corresponding closed linear operator $L: L_{2, m} \rightarrow L_{2 . m^{\prime}}$ be coercive. Then $L$ is nniformly elliptic in $R^{n}$.

Proor. - The proof is exactly analogous to a proof of a converse of Garding's inequality due to $\mathrm{S}$. Agmon [3, p. 86]. Therefore the proof is only skecthed here, to show how it applies to systems.

By assumption, there exists a constant $K$ such that (3.16) holds for all $u \in D(L)$. Agmon's method is to take, in (3.16),

$$
u(x)=e^{i t_{p} \cdot x} \psi(x)
$$

where $t \in R, p \in R^{n}$ and $\psi \in C_{0}^{1}\left(R^{n} ; C^{m}\right)$, and then make $t \rightarrow \infty$. A short calcu. lation gives

$$
\|u\|_{1}^{2} \sim t^{2}|p|^{2} \int_{R^{n}}|\psi(x)|^{2} d x, \quad t \rightarrow \infty,
$$

$$
\|L u\|^{2} \sim t^{2} \int_{R^{n}} \mid L(p, x) \biguplus(x){ }^{2} d x, t \rightarrow \infty
$$


which, with (3.16), implies

$$
|p|^{2} \int_{R^{n}}|\psi(x)|^{2} d x \leq K^{2} \int_{R^{n}}|L(p, x) \psi(x)|^{2} d x
$$

for all $\psi \in C_{0}^{1}\left(R^{n} ; C^{m}\right)$. Now take

$$
\psi(x)=j_{k}\left(x-x_{0}\right)^{1 / 2} v
$$

where $j_{k}(x)$ is the mollifier (2.18) and $v$ is a constant vector. Making $k \rightarrow \infty$ and using the continuity of $L(p, x)$ gives

$$
|p|^{2}|v|^{2} \leq K^{2}\left|L\left(p, x_{0}\right) v\right|^{2}
$$

Since $x_{0}$ is an arbitrary point in $R^{n}$, this proves that $L$ is uniformly elliptic.

\section{§4. - Coerciveness Inequalities for Nonelliptic Operators of Constant Deficit.}

In this section and the next coerciveness inequalities on subspaces (Definition 1.4) are derived for nonelliptic differential operators $L$ of constant deficit $k$ on $R^{n}$ (Definition 1.5). The examples in $\$ 1$ show how such inequalities can be found by constructing an augmented operator

$$
L^{\prime \prime}=\left(\begin{array}{l}
L^{\prime} \\
L
\end{array}\right)
$$

which is coercive. It is shown beloy that suoh augmented operators $L^{\prime \prime}$ always exist, although $L^{\prime}$ may not be a first-order differential operator. The case in which $L^{\prime}$ is a first-order differential operator is treated in this section. The more general case is treated in $\$ 5$ for the case of operators

$$
\Lambda=-i E(x)^{-1} \sum_{j=1}^{n} A_{j} D_{j}
$$

which are perturbations of uniformly propagative operators.

Defrnirion 4.1. - Let $L^{\prime}: L_{2, m} \rightarrow L_{2, k}$ be an (unbounded) linear operator (not necessarily a differential operator). Let $L^{\prime \prime}$ be the operator with domain $D\left(L^{\prime \prime}\right)=D\left(L^{\prime}\right) \cap D(L)$ defined by $(4.1)$. Then $L^{\prime \prime}$ is called an augmentation of the operator $L$. 
DEFinirion 4.2. - An augmentation $L^{\prime \prime}$ of an operator $L$ is said to be $L$-coercive if and only if $D\left(L^{\prime \prime}\right)=L_{2, m}^{1}$ and there exists a constant $K>0$ such that

$$
\left.\|u\|_{1}^{2} \leq K^{2}\left(\left\|L^{\prime \prime} u\right\|^{2}+\|u\|^{2}\right\rangle \text { for all } u \in D^{\prime} L^{\prime \prime}\right)
$$

that is

$$
\|u\|_{1}^{2} \leq K^{2}\left(\|L u\|^{2}+\|L u\|^{2}+\|u\|^{2}\right)
$$

$$
\text { for all } u \in D\left(L^{\prime}\right) \cap D(L) \text {. }
$$

Note that if $L$ has an augmentation which is $L$-coercive then $L$ is coercive on the subspace $N\left(L^{\prime}\right)$ :

$$
\|u\|_{1}^{2} \leq K^{2}\left(\|L u\|^{2}+\|u\|^{2}\right) \text { for all } \quad u \in D(L) \cap N\left(L^{\prime}\right) .
$$

Let $L^{\prime}$ be a $k \times m$ matrix differential operator of order $x$ :

$$
L^{\prime}=\sum_{|x|=x} L_{x}^{\prime}(x)(-i D)^{\alpha}
$$

The standard multi-index notation is used in (4.6); i.e. $\alpha=\left(\alpha_{1}, \ldots, \alpha_{n}\right)$ is an $n$-tuple of non-negative integers and $|\alpha|=\alpha_{1}+\ldots+\alpha_{n}$, while $(-i D)^{\alpha}=\left(-i D_{1}\right)_{1}^{\alpha_{1}} \ldots\left(-i D_{n}\right)^{\alpha_{n}}$. The symbol of $L^{\prime}$ is defined by

$$
L^{\prime}(p, x)=\sum_{|\alpha|=x_{t}} L_{2}^{\prime}(x) p^{\alpha}, p \in R^{n}
$$

where $p^{x}=p_{1}^{x_{1}} \ldots p_{n}^{\alpha_{n}}$. The symbol of the augmented operator $L^{\prime \prime}$ is

$$
L^{\prime \prime}(p, x)=\left(\begin{array}{l}
L^{\prime}(p, x) \\
L(p, x)
\end{array}\right)
$$

Definimion 4.3. - The augmented operator $L^{\prime \prime}$ is elliptic at $x_{0} \in R^{n}$ if and only if

$$
\text { rank } L^{\prime \prime}\left(p, x_{0}\right)=m \text { for all } p \in R^{n}-: 0 ! \text {, }
$$

$L^{\prime \prime}$ is elliptic in a region $\Omega \subset R^{n}$ if and only if it is elliptic at each $x_{0} \in R^{n}$.

This definition generalizes Definition 1.3. It is also a generalization to overdetermined systems of a definition of ellipticity due to $A$. Dougris and Nirenberg [9] which has been used as a basis for a priori estimates for elliptic systems by S. Agmon, A. Douglis and L. Nirenberg [4]. It is shown here that every constant deficit operator $L$ has an elliptic augmentation in 
J. R. Schulenberger - C. H. Wilcox: Coerciveness inequalities, etc.

this sense. In order to achieve this, $L^{\prime}$ is required to satisfy the following conditions which are suggested by the examples of $\S 1$ :

$$
\text { rank } L^{\prime}(p, x)=k \text { for all } p \in R^{n}-\{0\} \text { and } x \in R^{n} \text {, }
$$

where $k$ is the deficit of $L$.

$$
L^{\prime}(p, x) M(x) L(p, x)^{*}=0 \text { for all } p \in R^{n} \text { and } x \in R^{n}
$$

where, for each $x \in R^{n}, M(x)$ is a Herminas positive definite matrix.

Leman 4.1. - If $L$ is an operator of constant deficit $k$ in $R^{n}$ and $L^{\prime}$ satisfies (4.10) and (4.11) then the augmented operator $L^{\prime \prime}$ is elliptie in $R^{n}$.

Proof. - (4.11) implies that the rows of $L^{\prime}(p, x)$ and $L(p, x)$ are orthogonal in the sense of the $H_{\text {ermitian }}$ inner product $(\xi, \eta)=\xi^{*} M(x) \eta$. Hence the rows of $L^{\prime}(p, x)$ are linearly independent of the rows of $L(p, x)$, and therefore

$$
\operatorname{rank} L^{\prime \prime}(p, x)=\operatorname{rank} L^{\prime}(p, x)+\operatorname{rank} L(p, x)=m .
$$

Theorem 4.2. - Let $L$ have constant deficit $k$ in $R^{n}$ and for each $x \in R^{n}$ let $M(x)$ be an $m \times m$ Hermitian positive definite Hermitian matrix. Then there exists an operator $L^{\prime}$ of the form (4.6) which satisfies (4.10) and (4.11). In particular, the augmented operator $L^{\prime \prime}$ is elliptic in $R^{n}$.

Proof, - A simple computation shows that (4.11) is equivalent to the condition

$$
L(p, x) M(x) L^{\prime}(p, x)^{*}=0 \text { for all } p \in R^{n} \text { and } x \in R^{n} .
$$

This equation states that the rows of $L^{\prime}(p, x)$ are solutions of the homogeneous linear system

$$
(L(p, x) M(x)) u=0 .
$$

Since $M(x)$ is nonsingular, rank $L(p, x) M(x)=\operatorname{rank} L(p, x)=m-k$.

Thus the nullspace of $L(p, x) M(x)$ has dimension $k$. Hence (4.14) has $k$ linearly independent solutions. Moreover, such solutions can be constructed as homogeneous rational functions of the components of $L(p, x) M(x)$; e.g. by Gaussian elimination. Multiplying these solutions by their least common denominators gives solutions which are homogeneous polynomials in the components of $L(p, x) M(x)$. Hence there are $k$ linearly independent solutions of this form. Define $L^{\prime}(p, x)$ to be a $k \times m$ matrix whose rows are these solutions. Then $L^{\prime}(p, x)$ has properties $(4.10)$ and $(4.11)$ and is the symbol of an operator $L^{\prime}$ of the form (4.6). 
Corollary 4.3. - If $L$ has coefficients $L_{1}(x), \ldots, L_{n}(x) \in C^{s}\left(R^{n}\right)$ and $M(x) \in C^{s}\left(R^{n}\right)$ then $L^{\prime}$ has coefficients $L_{x}^{\prime}(x) \in C^{s}\left(R^{n}\right)$.

Proof. - The coefficients $L_{\alpha}^{\prime}(x)$ are polynomials in the coefficients of $L$ and $M(x)$.

For operators $L$ of constant deficit having an elliptic angmentation $L^{\prime \prime}$ which is a first-order operator the following coerciveness theorem holds.

Theonem 4.4. - Let $L$ satisfy hypotheses (2.1), (2.2) and have constant deficit $k$ in $R^{n}$. Let $L$ have an elliptic augmentation $L^{\prime \prime}$ which is a first-order operator and satisfies $(4.11)$ where $M(x)$ is a given function. Assume that

$$
\lim _{|x| \rightarrow \infty} L_{j}(x)=L_{j}^{\infty}, j=1,2, \ldots, n
$$

uniformly in $x /|x|$ where

$$
L_{\infty}=i \sum_{j=1}^{n} L_{j}^{\infty} D_{j}
$$

has constant deficit $k$. Moreover, assume that $M(x)$ and $D_{j} M(x)$ are continuous and bounded in $R^{n}$ and

$$
\lim _{|x| \rightarrow \infty} M(x)=M_{\infty}
$$

uniformly in $x /|x|$ where $M_{\infty}$ is positive definite. Then $L^{\prime \prime}$ is uniformly elli. ptic on $R^{n}$ and hence $L$ is coercive on $N\left(L^{\prime}\right)$.

Proop, - The components of $L^{\prime}(p, x)$ are polynomials in the components of $L(p, x)$ and $M(x)$. Thus $L^{\prime \prime}$ satisfies the hypotheses of Lemma 3.3 and hence is uniformly elliptic on $R^{n}$. The last conclusion follows from Theorem 3.5 and Definition 4.2 .

If a bounded and uniformly positive definite matrix-valued function $E(x)$ is given on $R^{n}$ then the HILBERT space $H=L_{2, m}(E)$ is defined and it is meaningful to ask if $L$ is coercive on the sub-space $N(L)^{1}$, the orthogonal complement of $N(L)$ in $H$. Note that the angmentation $L^{\prime \prime}$ and the subspace $N\left(L^{\prime}\right)$ of Theorem 4.2 depend on the choice of the matrix-valned function $M(x)$, and it may be possible to choose $M(x)$ so that $N(L)^{\perp} \subset N\left(L^{\prime}\right)$. This is carried out in $\$ 5$ for perturbations of uniformly propagative systems. To see how it can be done recall that (cf. (1.27))

$$
\overline{R\left(L^{*}\right)}=N(L)^{\perp}
$$


J. R. Schulenberger - C. H. Wilcox: Coerciveness inequalities, etc. 263

where $L^{*}$ is the adjoint of $L$ on $H$. Thus, $N(L)^{1} \subset N\left(L^{\prime}\right)$ provided that

$$
L^{\prime} L^{*}=0
$$

which implies that $\overline{R\left(L^{*}\right)} \subset N\left(L^{\prime}\right)$. In particular, if $L$ is selfadjoint the condition becomes $L^{\prime} L=0$. In $\S 5$,

$$
\Lambda=E(x)^{-1} A \quad \text { where } \quad A=-i \sum_{j=1}^{n} A_{j} D_{j}
$$

has constant coefficients. Define $A^{\prime}$ by the condition $A^{\prime}(p) A(p)=0$, as in Theorem 4.2, so that $A^{\prime} A=0$. Then defining $\Lambda^{\prime}$ by

$$
\Lambda^{\prime}=A^{\prime} E(x)
$$

gives $\Lambda^{\prime} \Lambda=A^{\prime} A=0$ and $N(\Lambda)^{\perp} \subset N\left(\Lambda^{\prime}\right)$.

\section{\$ 5. - Coerciveness Inequalities for Perturbations of Uniformly Propa- gative System.}

This section deals with operators of the form

$$
\Lambda=i E(x)^{-1} \sum_{j=1}^{n} A_{j} D_{j}
$$

which occur in wave propagation theories of classical physics. It is assumed, as in $\S 1$, that $E(x), A_{1}, \ldots, A_{n}$ are Hermimin, the $A_{j}$ are constant and $E(x)$ is bounded and uniformly positive definite (i.e., satisfies (1.5)). It is also as. sumed that $\Lambda$ is homogeneous at infinity in the sense of the following

Definition 5.1. $-\Lambda$ is a said to be homogeneous at infinity if and only if there exists a positive definite $E_{0}$ such that

$$
\lim _{|x| \rightarrow \infty} E(x)=E_{0}, \quad \text { uniformly in } x /|x| .
$$

The operator

$$
\Lambda_{0}=i E_{0}^{-1} \sum_{j=1}^{n} A_{j} D_{j}
$$

which has constant coefficients, is called the unperturbed operator and $\Lambda$ is referred to as a perturbation of $\Lambda_{0}$.

The purpose of this section is to prove that the operator $\Lambda$ on $H$ is coercive on the subspace $N(\Lambda)^{\perp}$ provided that the unperturbed system $\Lambda_{0}$ is 
uniformly propagative. To begin, the definitions and properties of these systems are reviewed briefly. Proofs of the properties are contained in $[6,22$, $23,24]$ and are not repeated here.

$\Lambda_{0}$ has the symbol

$$
\Lambda_{0}(p)=E_{0}^{-1} A(p) \text { where } A(p)=\sum_{j=1}^{n} A_{j} p_{j} .
$$

The characteristic polynomial of $\Lambda_{0}(p)$ is

$$
P(p, \lambda)=\operatorname{det}\left(\lambda I-\Lambda_{0}(p)\right)
$$

and has a factorization

$$
P(\boldsymbol{p}, \lambda)=Q_{1}^{m_{1}}(\boldsymbol{p}, \lambda) \ldots Q_{n}^{m_{N}}(\boldsymbol{p}, \lambda)
$$

where the factors $Q_{j}(p, \lambda)$ are distinct homogeneous polynomials in $(p, \lambda)$, irreducible over the complex number field. The polynomial

$$
Q(p, \lambda)=Q_{1}(p, \lambda) \ldots Q_{N}(p, \lambda)
$$

is known to be the minimal polynomial for $\Lambda_{0}(p)$ (see [22, p. 56] and [6, p. 87]).

Definition 5.2. - The operator $\Lambda_{0}$ is said to be uniformly propagative if and only if

(1) The locus $S=\{p: Q(p, 1)=0\}$ is bounded in $R^{n}$, and

(2) $p \cdot \nabla_{p} Q(p, 1)=p_{1} D_{1} Q(p, 1)+\ldots+p_{n} D_{n} Q(p, 1) \neq 0$ when $p \in S$.

$Q(p, \lambda)$ can be written as a polynomial in $\lambda(4)$

$$
Q(p, \lambda)=\lambda^{2}+Q_{1}(p) \lambda^{i-1}+\ldots+Q_{l-1}(p) \lambda+Q(p) .
$$

The roots $\lambda_{j}(p)$ of $Q(p, \lambda)=0$ are the propagation speeds of plane waves governed by the operator $\Lambda_{0}$. Their behavior is described by

Theorem 5.1. - For uniformly propagative systems

(1) $Q(p, \lambda)=0$ has $l$ distinct real roots $\lambda_{j}(p)$ for each $p \in R^{n}-\{0 \mid$.

(2) The roots can be enumerated to form $l$ distinct branches $\lambda_{j}(p)$ which are analytic for all $p \in R^{n}-\{0\}$.

(3) Each $\lambda_{j}(p)$ has constant algebraic sign for all $p \in R^{n}-\{0\}$. In particular, a root $\lambda_{j}(p)$ vanishes for some $p \in R^{n}-\{0\}$ if and only if it vanishes identically.

(4) Note that the coefficients $Q_{j}(p)$ in $(58)$ are different from the factors $Q_{j}(p, \lambda)$ in $(5.7)$. 
Corollary 5.2. - The roots $\lambda_{l}(p)$ can be enumerated as follows:

Case 1. $-l=2 \rho$. The roots are indexed by the $2 \rho$ indices $l \pm 1, \pm 2$, $\ldots \pm \rho\}$ and

$$
\lambda_{p}(p)>\lambda_{p-1}(p)>\ldots>\lambda_{1}(p)>0>\lambda_{-1}(p)>\ldots>\lambda_{-p}(p)
$$

for all $p \in R^{n}-, 0$ !

Case 2. $-l=2 p+1$. The roots are indexed by the $2 \rho+1$ indices $\{0, \pm 1, \pm 2, \ldots, \pm \rho\}$ and

$$
\lambda_{\rho}(p)>\ldots>\lambda_{1}(p)>0 \equiv \lambda_{0}(p)>\lambda_{-1}(p)>\ldots>\lambda_{-\rho}(p)
$$

for all $p \in R^{n}-\{0\}$. Moreover, with this enumeration the roots satisfy the relations

$$
\lambda_{-j}(p)=\lambda_{j}(-p) \text { for } j= \pm 1, \ldots, \pm \rho .
$$

The roots $\lambda_{j}(p)$ induce an orthogonal direct sum decomposition of the Hurbert space $H_{0}$ which reduces the selfadjoint operator $\Lambda_{0}$ on $H_{0}$ associated with (5.3). The decomposition is defined as follows (see [24] for details).

$$
\left.\left(\lambda I-\Lambda_{0} p\right)\right)^{-1}=\sum_{\mid k=\pi(l)}^{p} \widehat{P}_{k}(p)\left[\lambda-\left.\lambda_{k}(p)\right|^{-1}\right.
$$

where $\pi(l)=1$ if $l=2_{c}$ and $\pi(l)=0$ if $l=2 o+1$. The matrices $\widehat{P}_{k}(p)$ are homogeneous functions of degree zero which are analytic for $|p|=1$ and satisfy $\sum_{|k|=\pi(l)}^{\stackrel{p}{ }} \widehat{P}_{k}(p)=I, \widehat{P}_{j}(p) \widehat{P}_{k}(p)=\delta_{j k} \widehat{P}_{k}(p)$, and $\left(E_{0} \widehat{P}_{k}(p)\right)^{*}=E_{0} \widehat{P}_{k}(p)$. These properties imply that the $\widehat{P}_{k}(p)$ form a complete family of orthogonal projections in the unitary space $C_{E_{0}}^{m}$ with inner product $(\xi, \eta)=\xi^{*} E_{0} \eta$, and $\widehat{P}_{k}(p)$ is the projection onto the eigenspace of $\Lambda_{0}(p)$ for the eigenvalue $\lambda_{2}(p)$. Corresponding operators $P_{k}$ on $H_{0}$ are defined by means of the Fourier transform $\Phi$ defined by (1.19) above:

$$
P_{k}=\Phi^{*} \ddot{P}_{k} \Phi
$$

The properties of the $P_{k}$ are described by

LemMa 5.3. - The operators $P_{k},|k|=\pi(l), \ldots, \rho$, form a complete set of orthogonal projections on $H_{0}$; i.e.,

$$
\sum_{|k|=\pi(l)}^{p} P_{k}=I
$$




$$
P_{j} P_{k}=\delta_{j k} P_{k} \quad \text { for } \quad \pi(l) \leq|j|,|k| \leq p
$$

and

$$
P_{k}^{*}=P_{k} \quad \text { for } \quad \pi(l) \leq|k| \leq \rho .
$$

Corollary 5.4. - If $H_{0}^{k}=P_{k} H_{0}$, then

$$
H_{0}=\underset{\mid k,=\pi(l)}{\oplus} H_{0}^{k}
$$

Moreover $\Lambda_{0}$ commutes with the $P_{k}$ and hence is reduced by the decomposition (5.17).

In Case 2 of Corollary $5.2(l=2 \rho+1)$ the projection $P_{0}$ exists and is the orthogonal projection onto $N\left(\Lambda_{0}\right)$, the null space of $\Lambda_{0} . P_{0}$ plays an important part in the proof of the coercireness inequality for perturbations of uniformly propagative systems. Since $\widehat{P}_{0}(p)$ is homogeneous of degree zero and $\widehat{P}_{0}(\eta)$ is analytic on the unit sphere $\Omega=\{\eta:|\eta|=1\}$ it follows from results of A. CALDeron and A. Z Z p. 116] that $P_{0}=\Phi^{*} \widehat{P}_{0} \Phi$ is a singular integral operator of the form

$$
P_{0}=\varkappa_{0}+\tilde{P}_{0}
$$

where $x_{0}$ is the constant matrix operator

$$
\left.x_{0}=\Omega_{n}^{-1} \int_{0} \widehat{P}_{0}^{\prime} \eta\right) d \Omega_{\eta}
$$

( $d \Omega_{\eta}$ is the element of hyperarea on $\Omega$ and $\Omega_{n}$ is the hyperarea of $\Omega$.), and $\tilde{P}_{0}: H_{0} \rightarrow H_{0}$ is a singular integral operator of the form

$$
\left.\tilde{P}_{0} f(x)=\int_{R^{n}} \Gamma_{0}{ }^{\prime} \eta\right)|x-y|^{-n} f(y) d y, \quad \eta=\frac{x-y}{|x-y|} .
$$

The right side of $(5.20)$ is a singular integral, defined as the limit in $H_{0}$ of

$$
\int_{\mid x-y \geq \varepsilon} \Gamma_{0}(\eta)|x-y|^{-n} f(y) d y
$$

$\Gamma_{o}(\eta)$ is a matrix-valued analytic function with mean zero on $\Omega$ :

$$
\int_{\Omega} \Gamma_{0}(\eta) d \Omega_{n}=0
$$


J. R. Schulenberger - C. H. Wilcox: Coerciveness inequalities, etc. 267

The defining equation for $\Gamma_{0}(\eta),(5.20)$, is equivalent to the condition that $|x|^{-n} \Gamma_{0}(x /|x|)$ is the inverse Fourier transform of $\widehat{P}_{0}(p)-x_{0} I$ in the sense of distribution theory:

$$
\left.\frac{\Gamma_{0}(x /|x|)}{|x|^{t}}=D^{*} \cdot \widehat{P}_{0}-x_{0} I\right)(x)
$$

A discussion of $P_{0}$ and its relation to tie singularity of the resolvent keruel for $\Lambda_{0}$ may be found in [19].

The differential operators (5.1) and (5.3) determine unique selfadjoint extensions $\Lambda$ and $\Lambda_{0}$, on $H$ and $H_{0}$ respectively, whose domains may be defined as in [22] or as in $\$ 2$ above (Theorem 2.6 and Corollary 2.7). The perturbed and unperturbed ScHröprager equations (1.12) corresponding to $\Lambda$ and $\Lambda_{0}$ have static solutions if and only if zero is in the point spectrum of $\Lambda$ and $\Lambda_{0}$. This is true precisely in Case 2 of Corollary 5.2, when zero is a root of $Q(p, \lambda)$. The operators $\Lambda$ and $\Lambda_{0}$ are nonelliptic if and only if zero is in their point spectra. The situation is described by

Lemma 5.5. - Let $\Lambda_{0}$ be uniformly propagative. Then

Case 1. $-\Lambda$ and $\Lambda_{0}$ are elliptic if and only if $\lambda=0$ is not a root of $Q(p, \lambda)$

Case 2. - $\Lambda$ and $\Lambda_{0}$ are nonelliptic operators of constant deficit $k$ in $R^{n}$ if and only if $\lambda=0$ is a root of $Q ; p, \lambda)$ and $k$ is the multiplicity of $\lambda=0$ as a root of $P(p, \lambda)$.

Proop. - In Case $1, \lambda=0$ is not a root of $Q(p, \lambda)$ or $P(p, \lambda)$. Hence $\Lambda_{0}(p)$ is nonsingular waich implies that rank $\Lambda_{0}(p)=m$; i.e., $\Lambda_{0}$ is elliptic. Moreover, the symbol for $\Lambda$ can be written

$$
\Lambda(p, x)=E(x)^{-1} \sum_{j=1}^{n} A_{j} p_{j}=E(x)^{-1} E_{0} \Lambda_{0}(p)
$$

Thus, since $E(x)$ and $E_{0}$ are nonsingular, rank $\Lambda(p, x)=m$ and $\Lambda$ is elliptic.

In Case $2, \lambda=0$ is a simple root of $Q(p, \lambda)$; i.e., $Q_{j}(p, \lambda)=\lambda$ is one of the irreducible factors of $P(p, \lambda)$. Thus $P(p, \lambda)$ has a factor $\lambda^{k}$ where $k \geq 1$. Hence $\lambda=0$ is an eigenvalue of $\Lambda_{0}(p)$ of multiplicty $k$. It is easy to verify that $\left.\Lambda_{0}{ }^{\prime} p\right)$ is selfadjoint on the inner product space with product $(\xi, \eta)=\xi^{*} E_{0} \eta$. Hence the nullspace of $\Lambda_{0}(p)$ has dimension $k$ and it follows that

$$
\operatorname{rank} \Lambda(p, x)=\operatorname{rank} \Lambda_{0}(p)=m-\not b ;
$$

i.e., $\Lambda$ and $\Lambda_{0}$ are nonelliptic operators of constant deficit $k$ in $R^{n}$. 
Coerciveness inequalities for perturbations of uniformly propagative systems will now be derived, on the basis of the preliminary results given above. Two special cases are treated in Theorems 5.6 and 5.7. The general case is given in Theorem 5.8 .

Theorem 5.6. - Let $\Lambda$ be an operator of the form (5.1) such that $E(x)$ and $D_{j} E(x), j=1, \ldots, n$, are continnons and bounded on $R^{n}$ and $E(x)$ satisfies (1.5). Assume that $\Lambda$ is homogeneons at infinity with a limit operator $\Lambda_{0}$ which is uniformly propagative and does not have zero as a point eigenvalue. Then $\Lambda$ is coercive on $H$.

Proor. - Under the hypotheses of the theorem, Lemma 3.3 is applicable to $A$. Hence $A$ is uniformly elliptic on $R^{n}$ and it follows by Theorem 3.5 that $\Lambda$ is coercive on $H$.

Next let $\Lambda_{0}=-i E_{0}^{-1} \sum_{j=1}^{n} A_{j} D_{j}$ be a aniformly propagative operator with constant deficit $k>0$. Then the operator

$$
A=-i \sum_{j=1}^{n} A_{j} D_{j}
$$

has constant deficit $k$. Let $A^{\prime}$ be the corresponding operator defined by Theorem 4.2 with $M(x)=I$, such that

$$
A^{\prime \prime}=\left(\begin{array}{l}
A^{\prime} \\
A
\end{array}\right)
$$

is elliptic and (condition (4.11))

$$
A^{\prime}(p) A(p)^{*}=A^{\prime}(p) A(p)=0 \text { for' all } p \in R^{n}
$$

It follows that the operators $A$ and $A^{\prime}$ satisfy

$$
A^{\prime} A=0
$$

In the special case that $A^{\prime}$ is a first order operator, i.e.,

$$
A^{\prime}(p)=\sum_{j=1}^{n} \quad f_{j}^{\prime} p_{j}
$$

the coerciveness of $\Lambda$ on $N(\Lambda) \perp$ can be deduced from Theorem 4.4. To see this define an augmenting operator $\Lambda^{\prime}$ for $\Lambda$ by

$$
\Lambda^{\prime}=A^{\prime} E
$$


i.e.,

$$
\begin{aligned}
\Lambda^{\prime} u(x) & =-i \sum_{j=1}^{n} A_{j}^{\prime} D_{j}\{E(x) u(x)\} \\
& =-i \sum_{j=1}^{n} A_{j}^{\prime} E(x) D_{j} u(x)-i \sum_{j=1}^{n} A_{j}^{\prime}\left(D_{j} E(x)\right) u(x) .
\end{aligned}
$$

Note that

$$
\Lambda^{\prime}(p, x)=\sum_{j=1}^{n} A_{j}^{\prime} E(x) p_{j}=A^{\prime}(p) E(x)
$$

and

$$
\Lambda(p, x)=E(x)^{-1} A(p) \text { whence } \quad \Lambda(p, x)^{*}=A(p) E(x)^{-1}
$$

Thus

$$
\Lambda^{\prime}(p, x) E(x)^{-1} \Lambda(p, x)^{*}=A^{\prime}(p) A(p) E(x)^{-1}=0 .
$$

Thus condition (4.11) holds with $M(x)=E(x)^{-1}$. Moreoter,

$$
\Lambda^{\prime} \Lambda=A^{\prime} E\left(E^{-1} A\right)=A^{\prime} A=0 .
$$

This leads to

Theorem 5.7. - Let $\Lambda$ be an operator of the form 5.1$)$ such that $E(x)$ and $D_{j} E(x)$ are continuous and bounded on $R^{n}$ and $E(x)$ satisfies (1.5). Assume that $\Lambda$ is homogeneous at infinity with a limit operator $\Lambda_{0}$ which is uniformly propagative and has zero as a point eigenvalue, so that $\Lambda$ and $\Lambda_{0}$ are nonelliptic operators of constant deficit $k$ in $R^{n}$. Moreover, assume that there is a first order operator $A^{\prime}$ which satisfies (5.28). Then $\Lambda$ is coercive on $N(\Lambda)^{1}$.

Note that this result is applicable to a number of wave propagation equations of classical physics, including MaxweLL's equations and the equations of acoustics.

Proof of Theorem 5.7. - The hypotheses imply that $\Lambda$ satisfies the hypotheses of Theorem 4.4 with $M(x)=E(x)^{-1}$ and augmentation $\Lambda^{\prime}$ defined by (5.31). Hence, $\Lambda$ is coercive on $N\left(\Lambda^{\prime}\right)$. Moreover (5.36) implies that $N(\Lambda)^{1}=$ $=\overline{R(\Lambda)} \subset N\left(\Lambda^{\prime}\right)$, whence $\Lambda$ is coercive on $N(\Lambda)^{\prime}$.

The principle result in this paper is

Theonem 5.8. - Let $\Lambda$ be an operator of the form (5.1) such that $E(x)$ and $D_{j} E(x)$ are continuous and bounded on $R^{n}$ and $E(x)$ satisfies (1.5). Assume that $\Lambda$ is homogeneous at infinity with limiting operator $\Lambda_{0}$, which is uniformly propagative and has zero as a point eigenvalue, so that $\Lambda$ and $\Lambda_{0}$ are nonelliptic operators of constant deficit $k$ in $R^{n}$. Then $\Lambda$ is coercive on $N\left(\Lambda^{1}\right.$, . 
The remainder of this section is devoted to the proof of Theorem 5.8 . The seheme of the proof is the same as for Theorem 5.7. The proof is much more difficult than for Theorem 5.7 becanse it is impossible, in general, to find a first order differential operator $\Lambda^{\prime}$ such that $\Lambda^{\prime \prime}$ is elliptic. Instead, a $\Lambda^{\prime}$ which is not a differential operator is used, as was done for the equations of elasticity in $\$ 1$. Consequently, Theorem 35 for elliptic differential 3 operators cannot be used. Instead, the plan of the proof of Theorem 3.5 is carried out directly for the new operator $\Lambda^{\prime \prime}$.

The norm in the Sobourv space $L_{2, m}^{1}$ was defined by (cf. (3.15))

$$
\|u\|_{1}^{2}=\|u\|^{2}+\sum_{j=1}^{n}\left\|D_{j} u\right\|^{2}
$$

where $\|u\|$ denotes the norm in $L_{2, m}$. In what follows it is convenient to use the semi-norm $|u|_{\text {, defined by }}$

$$
|\boldsymbol{u}|_{1}^{2}=\sum_{j=1}^{n}\left\|D_{j} u\right\|^{2}
$$

Note that the coerciveness inequality (1.8) can be written

$$
|u|_{1}^{2} \leq K^{2}\left(\|L u\|^{2}+\|u\|^{2}\right)
$$

The construction of angmented operators for $\Lambda_{0}$ and $\Lambda$ is modeled after the treatment of the equations of elasticity in $\S 1$ To begin, the scalar ope. rator $(-\Delta)^{1 / 2}$ is defined as follows.

DEFinition 5.3. - $\left(-\Delta_{j}^{1 / 2}: H_{0} \rightarrow H_{0}\right.$ is the operator with domain $\left.D\left((-\Delta)^{1 / 2}\right\rangle=H_{0} \cap|u:| p \mid \widehat{u}(p) \in H_{0}\right\}$ such that $\left.\left((-\Delta)^{1 / 2} u\right)^{-}(p)\right)=|p| \widetilde{u}(p)$.

Note that $u \in D\left((-\Delta)^{1 / 2}\right)$ if and only if $u \in H_{0}$ and $|p|^{|2|}|\widetilde{u(p)}|^{2}=\left(p_{1}^{2}+\ldots\right.$ $\left.+p_{n}^{2}\right)|\widehat{u}(p)|^{2}$ is integrable. It follows by the Plas Heres theory that

$$
\left.D(-\Delta)^{1 / 2}\right)=L_{2, m}^{1} \text { and }\left\|(-\Delta)^{1 / 2} u\right\|^{2}=\sum_{j=1}^{n}\left\|D_{j} u\right\|^{2}
$$

for all $u \in D\left((-\Delta)^{1 / 2}\right)$.

Definition 5.4. - $\Lambda_{0}^{\prime}: H_{0} \rightarrow H_{0}$ is the operator with domain

$$
\left.D\left(\Lambda_{0}^{\prime}\right)=H_{0} \cap_{i} u: P_{0} u \in L_{2, m}^{1}\right\}
$$

such that

$$
\Lambda_{0}^{\prime}=(-\Delta)^{1 / 2} P_{0}
$$


J. R. Schulenberger - C. H. Wilcox: Coerciveness inequalities, etc.

Definimion 5.5. $-\Lambda^{\prime}: H \rightarrow H$ is the operator with domain

$$
D\left(\Lambda^{\prime}\right)=H \cap\left\{u: P_{0} J^{*} u \in L_{2, m}^{1}\right\}
$$

such that

$$
\Lambda^{\prime}=J \Lambda_{0}^{\prime} J^{*}
$$

Note that (5.40) implies that if $u \in D\left(\Lambda_{0}^{\prime}\right)$ then $P_{0} u \in D\left((-\Delta)^{1 / 2}\right)$ so that $\Lambda_{0}^{\prime} u$ is defined.

In (5.44), $J^{*}=E_{0}^{-1} J^{-1} E: H \rightarrow H_{0}$ is the adjoint of the identification map $J: H_{0} \rightarrow H$ defined by $(2.30)$.

Definimion 5.6. - The augmented operator $\Lambda^{\prime \prime}: H \rightarrow L_{2,2 m}$ is the operator with domain

$$
D\left(\Lambda^{\prime \prime}\right)=D(\Lambda) \cap D\left(\Lambda^{\prime}\right)
$$

such that

$$
\Lambda^{\prime \prime} u=\left(\begin{array}{c}
\Lambda^{\prime} u \\
\Lambda u
\end{array}\right)
$$

for all $u \in D\left(\Lambda^{\prime \prime}\right)$.

The notation (5.46) means that $\Lambda^{\prime \prime} u$ is a $2 m \times 1$ matrix-valued function for which the first $m$ components are those of $\Lambda^{\prime} u \in H$ and the last $m$ components are those of $\Lambda u \in H$. Since $H$ is equivalent to $L_{2, m}, \Lambda^{\prime \prime} u$ can clearly be identified with a vector in $L_{2,2 m}$.

Theorem 5.8 will be proved by showing that $\Lambda^{\prime \prime}$ is $\Lambda$-coercive (Definition 4.2) and coincides with $\Lambda$ on $N(\Lambda) \perp$ in $H$. The second property follows from

LEMMA 5.9. $-N\left(\Lambda_{0}\right)^{\perp} \subset N\left(\Lambda_{0}^{\prime}\right)$ and $N(\Delta)^{\perp} \subset N\left(\Lambda^{\prime}\right)$.

Proor. $-P_{0}$ is the orthogonal projection onto $N\left(\Lambda_{0}\right)$ in $H_{0}$.

Thus $N\left(\Lambda_{0}{ }^{1}{ }^{1}=N\left(P_{0}\right) \subset N(-\Delta)^{1 / 2} P_{0}\right)=N\left(\Delta_{0}^{\prime}\right)$. To prove the second relation note that (2.32) implies $\Lambda J=E^{-1} J E_{0} \Lambda_{0}$, whence $J N\left(\Lambda_{0}\right)=N(\Lambda)$. Moreover, $(u, J v)_{E}==\left(J^{*} u, v\right)_{E_{0}}$ for all $u \in H$ and $v \in H_{0}$. Combining these facts gives

$$
J^{*} N(\Lambda)^{\Perp}=N\left(\Lambda_{0}\right)^{\iota}
$$

Thus $J^{*} N(\Lambda) \perp=N\left(P_{0}\right)$, whence $N(\Lambda)^{\perp} \subset\left\{u: J^{*} u \in N\left(P_{0}\right) !=\left\{u: P_{0}\right.\right.$ $J^{*} u=0: \subset N\left(\Lambda^{\prime}\right)$

The symbols of the operators $\Lambda_{0}$ and $\Lambda$ are $\Lambda_{0}(p)=E_{0}^{-1} A(p)$ and $\Lambda(p, x)=E(x)^{-1} A(p)$, respectively, where $A(p)=\sum_{j=1}^{n} A_{j} p_{j}$. The symbols of
$\Lambda_{0}^{\prime}$ and $\Lambda^{\prime}$ are defined by

$$
\Lambda_{0}(p)={ }^{\prime} p \cdot \bar{P}_{v}(p)
$$


and

$$
\Lambda^{\prime}(p, x)=\Lambda_{0}^{\prime}(p) E_{0}^{-1} E(x)
$$

in keeping with (5.42) and (5.44). Finally, the symbol of $\Lambda^{\prime \prime}$ is defined by

$$
\Lambda^{\prime \prime}(p, x)=\left(\begin{array}{l}
\Lambda^{\prime}(p, x) \\
\Lambda(p, x)
\end{array}\right) .
$$

$\Lambda^{\prime \prime}$ is not a differential operator. However, it may be said to be elliptic in the sense of the following lemma (of. Lemma 3.1).

Lemma 5 10. - If $x \in R^{n}, p \in R^{n}-\{0\}$ and $\xi \in C^{m}$ then

$$
\Lambda^{\prime \prime}(p, x) \xi=0 \text { implies that } \xi=0 .
$$

Proof. $-\Lambda^{\prime \prime}(p, x \xi \xi=0$ implies that

$$
\Lambda^{\prime}(p, x) \xi=|p| \widehat{P}_{0}(p) E_{0}^{-1} E(x) \xi=0
$$

and

$$
\Lambda\left(p, x \xi \xi=E(x)^{-1} E_{0} \Lambda_{0}(p) \xi=0 .\right.
$$

Now if $p \in R^{n}-\left\{0^{\prime}, \widehat{P}_{0}(p)\right.$ is the orthogonal projection onto $N\left(\Lambda_{0}(p)\right)$ in the unitary space $C_{E_{0}}^{m}$ with inner product $(\xi, \eta)=\xi^{*} E_{0} \eta$ (cf. Lemma 5.3 and [24]). Thus (5.52) implies that $E_{0}{ }^{1} E(x) \xi \in N\left(P_{0}(p)\right)=N\left(\Lambda_{0}(p)\right){ }^{1}$ in $C_{E_{0}}^{m}$. Moreover, (5.53) implies that $\xi \in N(\Lambda(p))$. Thus

$$
0=\xi^{*} E_{0}\left(E_{0}^{-1} E(x) \xi\right)=\xi^{*} E(x) \xi,
$$

which implies that $\xi=0$ because $E(x)$ is positive definite.

Lems 5.11 . - Under the hypotheses of Theorem 5.8 there exists a con. stant $\mu>0$ such that

$$
\begin{aligned}
& \left|\Lambda^{\prime \prime}(p, x) u\right|^{2} \geq \mu^{2}|p|^{2} \mid u_{i}{ }^{2} \text { for all } \\
& p \in R^{n}, u \in C^{m} \text { and } x \in R^{n} .
\end{aligned}
$$

Proof. - Lemma 5.10 implies that for each $x_{0} \in R^{n}$ there exists a constant $\mu\left(x_{0}\right)$ such that $\left|\Lambda^{\prime \prime}\left(p, x_{0}\right) u\right|^{2} \geq\left.\mu\left(x_{0}\right)\right|^{2}|p|^{2} \mid u_{i^{2}}$ for all $p \in R^{n}$ and $u \in C^{m}$. The proof is the same as that of Lemma 3.2. The existence of a constant $\mu>0$, independent of $x$, such that (5.55) holds then follows as in the proof of Lemma 3.3 , because $E(x) \rightarrow E_{0}$ when $|x| \rightarrow \infty$, uniformly in $x /|x|$. 
It will now be shown that $\Lambda^{\prime \prime}$ is $\Lambda$-coercive under the hypotheses of Theorem 5.8. This will be done by a series of steps paralleling the proof of Theorem 3.5.

For any fixed $x_{0} \in R^{n}, \Lambda_{x_{0}}^{\prime \prime}$ is used to denote the operator with constant matrix coefficients obtained by making $x=x_{0}$ in the coefficients of $\Lambda^{\prime \prime}$. The domain of $\Lambda_{x_{0}}^{\prime \prime}$ is defined by means of the PLancheres theory, as for $\Lambda_{0}$ and $\Lambda_{0}^{\prime}$.

Lemma 5.12. - For each fixed $x_{0} \in R^{n}, \Lambda_{x_{0}}^{\prime \prime}$ is $\Lambda_{x_{0}}$-coercive and satisfies the inequality

$$
\left\|\Lambda_{x_{0}}^{\prime \prime} u\right\|^{2} \geq \mu^{2}|u|_{1}^{2}
$$

for all $u \in D\left(\Lambda_{x_{0}}^{\prime \prime}\right)$ where $\mu$ is the constant of Lemma 5.11 .

Proof. - $\|_{x_{0}}^{\prime \prime}$ has the symbol $\Lambda^{\prime \prime}\left(p, x_{0}\right)$. Hence (5.56) follows from (5.55) with $x=x_{0}$ by the Plancherel theory (cf. the proof of Lemma 3.6).

Next it is shown that $\Lambda^{\prime \prime}$ is coercive for all functions $u \in C_{0}^{1}\left(R^{n} ; C^{m}\right)$ whose support is sufficiently small (the analogue of Lemma 3.7).

Theorex 5.13. - Under the hypotheses of Theorem 58, there exist constant $\rho>0$ and $\mu_{0}>0$, depending only on $E(x)$ and $\Lambda_{0}$, such that

$$
|\varphi|_{1}^{2} \leq \mu_{0}^{2}\left(\left\|\Lambda^{\prime \prime} \varphi\right\|^{2}+\|\varphi\|^{2}\right)
$$

for all $\varphi \in C_{0}^{1}\left(R^{n} ; C^{m}\right)$ such that $\operatorname{supp} \varphi$ has diameter less than $\rho$.

Proof. - It follows from (5.2) and the continuity of $E(x)$ that $E(x)$ is uniformly continuons on $R^{n}$. Define $c=\max \left\{1, \max _{n \in Q}\left|\lambda_{ \pm}(\eta)\right|^{2}\right\}$ and define $\rho$ by the condition that

$$
\begin{aligned}
& \max : 2 \max _{\mid x-x^{0} \leq p}\left|E_{0}^{-1}\left[E(x)-E\left(x_{0}\right)\right]\right|^{2}, \\
& \left.c \max _{\left.\left|x-x_{0}\right| \leq p\right)}\left|\left[E(x)^{-1}-E\left(x_{0}\right)^{-1}\right] E_{0}\right|^{2}\right\}<\mu^{2} / 8
\end{aligned}
$$

where $\mu$ is the constant of Lemma 5.11. Let supp $\varphi \subset\left\{x:\left|x-x_{0}\right| \leq p:\right.$.

Then

$$
\begin{aligned}
\left\|\Lambda^{\prime \prime} \varphi\right\|^{2} & =\left\|\Lambda_{x_{0}}^{\prime \prime} \varphi+\left(\Lambda^{\prime \prime}-\Lambda_{x_{0}}^{\prime \prime}\right) \varphi\right\|^{2} \\
& \geq \frac{1}{2}\left\|\Lambda_{x_{\varphi}}^{\prime \prime} \varphi\right\|^{2}-\left\|\left(\Lambda^{\prime \prime}-\Lambda_{x_{0}}^{\prime \prime}\right) \varphi\right\|^{2} \\
& \geq\left(\mu^{2} / 2\right)|\varphi|_{1}^{2}-\left\|\left(\Lambda^{\prime \prime}-\Lambda_{x_{0}}^{\prime \prime}\right) \varphi\right\|^{2}
\end{aligned}
$$


274 J. R. Schulenberger - C. H. Wilcox: Coerciveness inequalities, etc.

by Lemma 5.13. Now

$$
\left\|\left(\Lambda^{\prime \prime}-\Lambda_{x_{0}}^{\prime \prime}\right) \varphi\right\|^{2}=\left\|\left(\Lambda^{\prime}-\Lambda_{x_{0}}^{\prime}\right) p\right\|^{2}+\left\|\left(\Lambda-\Lambda_{x_{0}}\right) \varphi\right\|^{2}
$$

and it remains to estimate the two terms on the right. First, since $\Lambda=E^{-1} E_{0} \Lambda_{0}$,

$$
\begin{aligned}
& \left\|\left(\Lambda-\Lambda_{x_{0}}\right) \varphi\right\|^{2}=\left\|\left(E^{-1}-E_{x_{0}}^{-1}\right) E_{0} \Lambda_{0} \varphi\right\|^{2} \\
& \quad \leq \max _{\left|x \rightarrow x_{0}\right| \leq \rho}\left|\left[E(x)^{-1}-E\left(x_{0}\right)^{-1}\right] E_{0}\right|^{2}\left\|\Lambda_{0} \varphi\right\|^{2} .
\end{aligned}
$$

Moreover, (cf. [24])

$$
\begin{aligned}
& \left\|\Lambda_{0} \varphi\right\|^{2}=\sum_{|k|=1}^{p}\left\|\lambda_{k}(\cdot) \widehat{P}_{k}^{2} \bar{\varphi}^{2} \leq\right\||\cdot| \widehat{P}_{0} \bar{\varphi}^{2}\left\|^{2}+\sum_{|k|=1}^{\rho}\right\| \lambda_{k}(\cdot) \widehat{P}_{k} \bar{\varphi} \|^{2}
\end{aligned}
$$

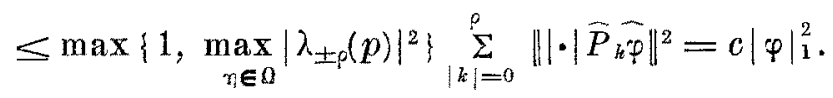

Combining (5.61) and (5.62) and using (5.48) gives

$$
\left\|\left(\Lambda-\Lambda_{x_{0}}\right) \varphi\right\|^{2} \leq\left(\mu^{2} / 8\right)|\varphi|_{1}^{2} .
$$

Next, since $P_{0}(-\Delta)^{1 / 2} C(-\Delta)^{1 / 2} P_{0}$ and $\left\|(-\Delta)^{1 / 2} u\right\|^{2}=|u|_{1}^{2}$ by $(5.40)$,

$\left\|\left(\Lambda^{\prime}-\Lambda_{x_{0}}^{\prime}\right) \varphi\right\|^{2}=\left\|\Lambda_{0}^{\prime}\left(E_{0}^{-1} E-E_{0}^{-1} E_{x_{0}}\right) \varphi\right\|^{2}$

$$
\begin{aligned}
& =\left\|(-\Delta)^{1 / 2} P_{0} E_{0}^{-1}\left(E-E_{x_{0}}\right) \varphi\right\|^{2}=\left\|P_{0}(-\Delta)^{1 / 2} E_{0}^{-1}\left(E-E_{x_{0}}\right) \varphi\right\|^{2} \\
& \leq\left\|(-\Delta)^{1 / 2} E_{0}^{-1}\left(E-E_{x_{0}}\right) \varphi\right\|^{2}=\sum_{j=1}^{n}\left\|D_{j} E_{0}^{-1}\left(E-E_{x_{0}}\right) \varphi\right\|^{2} \\
& \leq 2\left[\sum_{j=1}^{n}\left\|E_{0}^{-1}\left(D_{j} E\right) \varphi\right\|^{2}+\sum_{j=1}^{n}\left\|E_{0}^{-1}\left(E-E_{x_{0}}\right) D_{j} \varphi\right\|^{2}\right] \\
& \leq 2 n \max _{1 \leq j \leq n}\left\|E_{0}^{-1} D_{j} E\right\|_{\infty}^{2}\|\varphi\|^{2} \\
& +2 \max _{\left|x-x_{0}\right| \leq \rho}\left|E_{0}^{-1}\left(E(x)-E\left(x_{0}\right)\right)\right|^{2}|\varphi|_{1}^{2} \\
& \leq \mu_{1}^{2}\|\varphi\|^{2}+\left(\mu^{2} / 8\right)|\varphi|_{1}^{2}
\end{aligned}
$$

by (5.58), where $\mu_{1}^{2}=2 n \max _{1 \leq j \leq n}\left\|E_{0}^{-1} D_{j} E\right\|_{\infty}^{2}$ and $\|f\|_{\infty}$ denotes the maximum of $|f(x)|$ on $R^{n}$. Combining $(5.59),(5.60),(5.63)$ and $(5.64)$ gives

$$
\left\|\Lambda^{\prime \prime} \varphi\right\|^{2} \geq\left(\mu^{2} / 4\right)|\varphi|_{1}^{2}-\mu_{1}^{2}\|\varphi\|^{2} \text {. }
$$


Finally, if $\mu_{0}^{2}=\max \left(4 \mu^{-2}, 4 \mu_{1}^{2} \mu^{-2}\right)$ then (5.65) implies $(5.57)$, which completes the proof.

The next step is to remove the restriction that supp $\varphi$ has diameter less than $\rho$. The result is stated as

Theorem 5.14. - Under the hypotieses of Theorem 5.8, there exists a constant $\mu_{1}>0$, depending only on $E(x)$ and $\Lambda_{0}$ such that

$$
|\varphi|_{1}^{2} \leq \mu_{1}^{2}\left(\left\|\Lambda^{\prime \prime} \varphi\right\|^{2}+\|\varphi\|^{2}\right)
$$

for all $\varphi \in C_{0}^{1}\left(R^{n} ; C^{m}\right)$.

The proof makes use of the same partition of unity $\mid \zeta_{x}(x) !$ used in the proof of Theorem 35 (see (335)-3.38)). However, the proof is more complicated than for Theorem 3.5 because $\Lambda^{\prime}$ is not a differential operator. The following theorem is needed for the proof.

Theorem 5.15. - Let $B=E_{0}^{-1} E$. Then, under the hypotheses of Theorem 5.8 , there exists a constant $c_{0}>0$ such that

$$
\left|\sum_{a}\left\{\left\|\zeta_{\alpha} P_{0} B D_{j} \rho\right\|^{2}-\left\|P_{0} \zeta_{x} B D_{j} \varphi\right\|^{2}\right\}\right| \leq c_{0}\|\varphi\| \cdot\left\|D_{j} \varphi\right\|
$$

for all $\varphi \in C_{0}^{1}\left(R^{n} ; C^{m}\right)$ and $j=1,2, \ldots, n$.

Proof. - First note that

$$
\Sigma_{a}\left\|\zeta_{x} P_{0} B D_{j} \rho\right\|^{2}=\Sigma_{\alpha} \int_{R^{n}} \zeta_{x^{n}}^{2}\left(P_{0} B D_{j} \varphi\right)^{*} E_{0}\left(P_{0} B D_{j} \varphi\right) d x
$$

$$
=\int_{R^{n}}\left(\sum_{a} \zeta_{x}^{2}\right)\left(P_{0} B D_{j} \varphi\right)^{*} E_{0}\left(P_{0} B D_{j} \varphi\right) d x=\left\|P_{0} B D_{j} \varphi\right\|^{2}<\infty
$$

where the interchange of summation and integration is justified by LEBEsGUE's dominated convergence theorem. The other sum on the left in (5.67) is finite because $\varphi$ has compact support, so that $\zeta_{x} B D_{j} \varphi$ is zero for all but a finite number of $\alpha$. Now, since $P_{0}$ is an orthogonal projection on $H_{0}$,

$$
\begin{aligned}
& \left.\sum_{z} \quad\left\|\zeta_{\alpha} P_{0} B D_{j} p\right\|^{2}-\left\|P_{0} \zeta_{x} B D_{j} p\right\|^{2}\right\} \\
& =\sum_{\alpha}\left(\zeta_{x} P_{0} B D_{j} \rho, \zeta_{x} P_{0} B D_{j p} p\right)_{E_{0}}-\left(\zeta_{x} B D_{j} \uparrow, P_{0} \zeta_{x} B D_{j p}\right)_{E_{0}} \\
& =\sum_{a}\left(\zeta_{\alpha}\left(P_{0}-I\right) B D_{j} \varphi_{\varphi}, \zeta_{\alpha} P_{0} B D_{j} \varphi\right)_{E_{0}}+\left(\zeta_{\alpha} B D_{j} \varphi, \zeta_{x} P_{0} B D_{j} \varphi-P_{0} \zeta_{x} B D_{j} \xi\right)_{E_{v}} \\
& =\left(\sum_{x} \zeta_{x}^{2}\left(P_{0}-I\right) B D_{j} \varphi, P_{0} B D_{j} \varphi\right)_{E_{0}}
\end{aligned}
$$




$$
\begin{gathered}
+\sum_{\alpha}\left(\zeta_{x} B D_{j} \varphi, \zeta_{x} P_{0} B D_{j} \varphi-P_{0} \zeta_{\alpha} B D_{j} \varphi\right)_{E_{0}} \\
\left.=\left(P_{0}-I\right) B D_{j} \varphi, P_{0} B D_{j} \varphi\right)_{E_{0}}+\sum_{\alpha}\left(\zeta_{x} B D_{j} \varphi, \zeta_{\alpha} P_{0} B D_{j} \varphi-P_{0} \zeta_{\alpha} B D_{j} \varphi\right)_{E_{0}} \\
=\sum_{\alpha}\left(\chi_{\alpha} B D_{j} \varphi, \zeta_{x}\left[\zeta_{x} P_{0} B D_{j} \varphi-P_{0} \zeta_{x} B D_{j} \varphi\right]\right)_{E_{0}} .
\end{gathered}
$$

The first term in the next to the last expression is zero because $\left.P_{0}^{*}\left(P_{0}-I\right)=P_{0}^{\prime} P_{0}-I\right)=0 . \chi_{\alpha}$ is used to denote the characteristic function of supp $\zeta_{\alpha} \subset Q_{\alpha}$. Now $P_{0}=x_{0}+\tilde{P}_{0}$ (see $(5.18)$ ) where $\tilde{P}_{0}$ is a singular integral operator (5.20). When this is substituted in the last term of (5.69) the terms involving $x_{0}^{1}$ drop out because the scalar operator $\zeta_{x}$ commutes with $\varkappa_{0}$. Thus, if $\Pi_{a j}$ is defined by

$$
\Pi_{a j} p=\zeta_{\alpha} \tilde{P}_{0} B D_{j} \varphi^{i}-\tilde{P}_{0} \zeta_{x} B D_{j} \rho
$$

then $(5.20)$ implies that

$$
\Pi_{a j} f(x)=\int_{R^{n}} \frac{\Gamma_{0}(\eta)}{|x-y|^{n}}\left[\zeta_{x}(x)-\zeta_{x}(y)\right] B(y) D_{j} \ngtr(y) d y
$$

and, from (5.69),

$$
\begin{aligned}
& \sum_{\alpha} \quad\left\{\left\|\zeta_{x} P_{0} B D_{j} \varphi\right\|^{2}-\left\|P_{0} \zeta_{\alpha} B D_{j} \varphi\right\|^{2} \|\right. \\
& =\left|\sum_{\alpha}\left(\chi_{\alpha} B D_{j} \varphi, \zeta_{\alpha}\left[\zeta_{\alpha \alpha} \tilde{P}_{0} B D_{j} \varphi-\tilde{P}_{0} \zeta_{\alpha} B D_{j} \varphi\right]\right)_{E_{0}}\right| \\
& =\left|\quad \Sigma_{\alpha}\left(\chi_{\alpha} B D_{j} \varphi, \zeta_{\alpha} \Pi_{0, j}\right) E_{0}\right| \\
& \leq \int_{R^{n}} \Sigma_{\alpha}\left|\left(\chi_{\alpha} B D_{j \rho}\right)^{*} E_{0}\right|\left|\zeta_{\alpha} \Pi_{\alpha i p}\right| d x \\
& \left.\leq \int_{R^{q 4}}\left\{\sum_{\alpha}\left|\left(\chi_{\alpha} B D_{j} \rho\right)^{*} E_{0}\right|^{2}\right\}^{1 / 2}\right\} \quad \sum_{\alpha}\left|\zeta_{x} \Pi_{\alpha j p}\right|^{2} j^{1 / 2} d x \\
& \left.\leq\left.\left\{\int_{R^{n}} \Sigma_{\alpha} \mid \chi_{\alpha} B D_{j} \varphi\right)^{*} E_{0}\right|^{2} d x\right\}^{1 / 2}\left\{\int_{R^{\prime 2}} \Sigma_{\alpha}\left|\zeta_{\alpha} \Pi_{\alpha j} \varphi\right|^{2} d x\right\}^{1 / 2} \\
& =\left\{\sum_{\alpha} \int_{R^{n}} \chi_{\alpha}\left|\left(B D_{j} \varphi\right)^{*} E_{0}\right|^{2} d x\right\}^{1 / 2}\left\{\sum_{\alpha} \int_{R^{n}}\left|\zeta_{\alpha} \Pi_{\alpha j} \varphi\right|^{2} d x\right\}^{1 / 2} \\
& =\left\{2^{n} \int_{R^{n}}\left|\left(B D_{j} \varphi\right)^{*} E_{0}\right|^{2} d x\right\}^{1 / 2}\left\{\sum_{\alpha} \int_{R^{n}} \mid \zeta_{\alpha}\left[\left.I_{\alpha j p}\right|^{2} d x\right\}^{1 / 2}\right. \\
& \leq c_{1}\left\|D_{j} \varphi\right\|\left\{\sum_{\alpha} \int_{n}\left|\zeta_{\alpha} \Pi_{\alpha j \varphi}\right|^{2} d x\right\}^{1 / 2}
\end{aligned}
$$


where $c_{1}$ depends on $n$ and bounds for $E$ and $E_{0}$ only. Thus (5.67) will be proved if there exists a constant $c_{2}^{2}>0$ such that

$$
\sum_{\alpha} \int_{R^{n}}\left|\zeta_{\alpha} \Pi_{x j} \varphi\right|^{2} d x \leq c_{2}^{2}\|\varphi\|^{2}
$$

for all $\varphi \in C_{0}^{1}\left(R^{n} ; C^{u}\right)$.

To this end a now representation of $\Pi_{\alpha j}$ as a singular integral will be obtained. Consider the integral

$$
\int_{|x-y|>\varepsilon} \Gamma_{0}(\eta)|x-y|^{-\pi}\left[\zeta_{x}(x)-\zeta_{\alpha}(y)\right] B(y) D_{j} \varphi(y) d y
$$

which converges in $H_{0}$ to $\Pi_{\alpha j} \rho(x)$ when $\varepsilon \rightarrow 0$. Note that if $(5.74)$ is broken into two integrals containg $\zeta_{x}(x)$ and $\zeta_{x}(y)$ then each converges ${ }^{\prime}$ in $H_{0}$ and also pointwise almost everywhere to one of the terms on the right in (5.70) when $\varepsilon \rightarrow 0[7$, p. 290]. Moreover, (5.74) actually converges pointwise because the integrand is $O\left(r^{-n+1}\right)$ for $r=|x-y| \rightarrow 0$. I'hus the pointwise limit of (5.74) is equal a.e. to $\Pi_{x j} \varphi(x)$.

Integration by parts in (5.74) gives

$$
\begin{aligned}
\int_{r>\varepsilon} \mathfrak{l}_{0}(\eta) r^{-n}\left[\zeta_{x}(x)\right. & \left.\left.\left.-\zeta_{\alpha}(y)\right] B(y) D_{j} \varphi\right) y\right) d y \\
& =\int_{r>\varepsilon}\left(D_{x_{j}} \Gamma_{0}(\eta) r^{-n}\right)\left[\zeta_{x}(x)-\zeta_{\alpha}(y)\right] B(y) \varphi(y) d y \\
& -\zeta_{\alpha}(x) \int_{r>\varepsilon} \Gamma_{0}(\eta) r^{-n}\left[D_{j} B(y)\right] \varphi(y) d y \\
& +\int_{r>\varepsilon} \Gamma_{0}(\eta) r^{-n}\left[D_{j} \zeta_{x}(y) B(y)\right] \varphi(y) d y \\
& +\int_{r=} \eta \Gamma_{0}(\eta) r^{-n}\left[\zeta_{x}(x)-\zeta_{x}(y)\right] B(y) \varphi(y) d S_{y} \\
& \equiv I_{1}^{\varepsilon}(x)+I_{2}^{\varepsilon}(x)+I_{3}^{\varepsilon}(x)+I_{4,}^{\varepsilon}(x)
\end{aligned}
$$

in an obvious notation. Now $I_{2}^{\varepsilon}(x)$ and $I_{3}^{\Sigma}(x)$ converge in $H_{0}$ and pointwise a.e. [7]. It will be shown that $I_{1}^{\S}(x)$ and $I_{4}^{\mathfrak{\varepsilon}(x)}$ converge pointwise, from which it will follow that the right-hand side of $(5.75)$ converges to $\Pi_{\alpha j}^{s} \varphi(x)$ a.e. $\left(I_{1}^{\varepsilon}\right.$ and $I_{4}^{\varepsilon}$ also converge in $H_{0}$, but this fact is not needed here). First it is shown that the pointwise limit of $I_{1}^{\varepsilon}(x)$ exists. This is the content of the next two lemmas. 
LeMмa $5.16 .-$ For $k=1,2 . \ldots, n$

$$
\int_{0} r^{n+1} \eta_{k}\left(D_{x_{j}} \Gamma_{0}(\eta) r^{-n}, d \Omega_{n}=0\right.
$$

Proof. - Number the coordinates so that $x_{j}=x_{1}$ and introduce spherical coordinates as follows: $y_{1}=x_{1}+r \cos \theta_{1}, y_{2}=x_{2}+r \sin \theta_{1} \cos \theta_{2}, \ldots$, $y_{n-1}=x_{n-1}+r \sin \theta_{1} \ldots \sin \theta_{n-2} \cos \theta_{n-1}, y_{n}=x_{n}+r \sin \theta_{1} \ldots \sin \theta_{n-2} \sin$ $\theta_{n-1}$ where $-\pi \leq \theta_{n-1} \leq \pi$ and $0 \leq \theta_{k} \leq \pi$ for $k=1,2, \ldots, n-2$. Also note that $d \Omega=\sin ^{n-2} \theta_{1} \ldots \sin \theta_{n-2} d \theta_{1} \ldots d \theta_{n-1}$. Only $r$ and $\theta_{1}$ depend on $x_{1}$ and $D_{x_{1}} r=-\cos \theta_{1}, D_{x_{1}} \theta_{1}=r^{-1} \sin \theta_{1}$. Hence

$$
r^{n+1} D_{x_{1}}\left(\Gamma_{0}(\eta) r^{-n}\right)=n \cos \theta_{1} \Gamma_{0}(\eta)+\sin \theta_{1} D_{\theta_{1}} \Gamma_{0}(\eta) .
$$

Assume first that $\eta_{k}$ does not contain $\sin \theta_{1}$. Then $\eta_{k}=\eta_{1}=\cos \theta_{1}$ and

$$
r^{n+1} \eta_{k} D_{x_{1}}\left(\Gamma_{0}(\eta) r^{-n}\right)=\cos \theta_{1}\left[n \cos \theta_{1} \Gamma_{0}(\eta)+\sin \theta_{1} D_{\theta_{1}} \Gamma_{0}(\eta)\right] .
$$

Now a short calculation gives

$$
\begin{aligned}
& D_{\theta_{1}}\left[\sin ^{n-1} \theta_{1} \cos \theta_{1} \Gamma_{0}(\eta)\right] \\
& =\cos \theta_{1}\left[n \cos \theta_{1} \Gamma_{0}(\eta)+\sin \theta_{1} D_{\theta_{1}} \Gamma_{0}(\eta)\right] \sin ^{n-2} \theta_{1} \\
& -\sin ^{n-2} \theta_{1} \Gamma_{0}(\eta) .
\end{aligned}
$$

Equations (5.78) and (5.79) imply

$$
\begin{aligned}
& \int_{\Omega} r^{n+1} \eta_{1} D_{x_{1}}\left(\Gamma_{0}(\eta) r^{-n}\right) d \Omega_{\eta_{i}} \\
= & \int_{-\pi}^{\pi} d \theta_{n-1} \int_{0}^{\pi} \sin \theta_{n-2} d \theta_{n-2} \cdots \int_{0}^{\pi} \sin ^{n-3} \theta_{2} d \theta_{2} \\
& \cdot \int_{0}^{\pi} \sin ^{n-2} \theta_{1}\left\{\cos \theta_{1}\left[n \cos \theta_{1} \Gamma_{0}(\eta)+\sin \theta_{1} D_{\theta_{1}} \Gamma_{0}(\eta)\right\} d \theta_{1}\right. \\
= & \int_{-\pi}^{\pi} d \theta_{n-1} \cdot \int_{0}^{\pi}\left\{D_{\theta_{1}}\left[\sin ^{n-1} \theta_{1} \cos \theta_{1} \Gamma_{0}(\eta)\right]+\sin ^{n-2} \theta_{1} \Gamma_{0}(\eta) ! d \theta_{1}\right. \\
= & 0+\int_{\Omega} \Gamma_{0}(\eta) d \Omega_{n}=0,
\end{aligned}
$$

because $\Gamma_{0}^{\prime} \eta_{1}$ ) has mean zero on $\Omega$. This proves (5.76) for $\eta_{k}=\eta_{1}$. 
If $k \neq 1$ then $\eta_{k}$ contains a factor $\sin \theta_{1}$ and the result follows easily. Suppose, for example, that $k=3$. Then $\eta_{3}=\sin \theta_{1} \sin \theta_{2} \cos \theta_{3}$ and

$$
\begin{aligned}
& r^{n+1} \eta_{k} D_{x_{1}}\left(\Gamma_{0}(\eta) r^{-n}\right) \\
& =\cos \theta_{3} \sin \theta_{2} \sin \theta_{1}\left[n \cos \theta_{1} \Gamma_{0}(\eta)+\sin \theta_{1} D_{\theta_{1}} \Gamma_{0}(\eta)\right] .
\end{aligned}
$$

Bat

$$
\sin \theta_{1}\left[n \cos \theta_{1} \Gamma_{0}(\eta)+\sin \theta_{1} D_{\theta_{1}} \Gamma_{0}(\eta)\right] \sin ^{n-2} \theta_{1}
$$

$$
=D_{\theta_{1}}\left[\sin ^{n} \theta_{1} \mathrm{~T}_{0}(\eta)\right]
$$

whence

$$
\begin{aligned}
& \int_{\Omega} r^{n+1} \eta_{k} D_{x_{1}}\left(\Gamma_{0}(\eta) r^{-n} d \Omega_{\eta}\right. \\
= & \int_{-\pi}^{\pi} d \theta_{n-1} \cdots \int_{0}^{\pi} \cos \theta_{3} \sin \theta_{2} D_{\theta_{1}}\left[\sin ^{n} \theta_{1} \Gamma_{0}\left(\eta_{)}\right)\right] d \theta_{1}=0 .
\end{aligned}
$$

It is clear that a similar proof can be given for any $k \neq 1$.

Lemma 5.17. - Let $\psi(y)=B(y) \varphi(y)$. Then the singular integral

$$
\int_{R^{n}} r D_{x_{j}}\left(\Gamma_{0}(\eta) r^{-n}\right) \nabla \zeta_{\alpha}(x) \cdot \eta \psi(y) d y
$$

exists pointwise for $x \in R^{n}$.

Proof.

$$
\begin{aligned}
& \int_{\varepsilon<r<1} r D_{x_{j}}\left(\Gamma_{0}\left(\eta r^{-n}\right) \nabla \zeta_{\alpha}(x) \cdot \eta \psi(y) d y\right. \\
= & \left\{\int_{\Omega} \int_{\varepsilon}^{1}\left[r^{n+1} D_{x_{j}}\left(\Gamma_{0}(\eta) r^{-n}\right) \nabla \zeta_{\alpha}(x) \cdot \eta\right] r^{-1} d r d \Omega_{\eta}\right\} \psi(x) \\
+ & \int_{\varepsilon<r<1} r D_{x_{j}}\left(\Gamma_{0}(\eta) r^{-n}\right) \nabla \zeta_{x}(x) \cdot \eta[\psi(y)-\psi(x)] d y \\
= & \log \varepsilon^{-1}\left\{\sum_{k=1}^{n} D_{k} \zeta_{x}(x) \int_{\Omega} r^{n+1} \eta_{k} D_{x_{j}}\left(\Gamma_{0}(\eta) r^{-n}\right) d \Omega_{\eta}\right\} \psi(x) \\
+ & \int_{\varepsilon<\tau<1} r D_{x_{j}}\left(\Gamma_{0}(\eta) r^{-n}\right) \nabla \zeta_{\alpha}(x) \cdot \eta[\psi(y)-\psi(x)] d y .
\end{aligned}
$$


Now the coefficient of $\log \varepsilon^{-1}$ is zero by Lemma 5.16. Moreover, the integrand of the last integral is $O\left(r^{-n+1}\right)$ for $r \rightarrow 0$. This completes the proof of Lemma 5.17.

Coroliary 5.18. - The singular integral

$$
\int_{R^{n}}\left(D_{x_{j}}^{\sim} \Gamma_{0}(\eta) r^{-n}\right)\left[\zeta_{\alpha}(x)-\zeta_{\alpha}(y)\right] B(y) \varphi(y) d y
$$

exists as a pointwise limit.

This is an immediate consequence of Lemma 5.17 and the TArLor expansion of $\zeta_{\alpha}(y)$ about the point $x$. Next it will be shown that the pointwise limit of $\boldsymbol{I}_{4}^{\varepsilon}(x)$ exists.

Lexma 5.19. - The pointwise limit of $I_{4}^{\varepsilon}(x)$ exists and

$$
\lim _{\varepsilon \rightarrow 0} I_{q}^{\xi}(x)=-\left(\sum_{k=1}^{n} D_{k} \zeta_{\S}(x) \beta_{k}\right) B\left(x^{\prime} \varphi(x)\right.
$$

where

Proor,

$$
\beta_{k j}=\int_{\Omega} \eta_{k} \eta_{j} \Gamma_{0}(\eta) d \Omega_{\eta}
$$

$$
\begin{aligned}
I_{4}^{\varepsilon}(x) & \left.=\int_{r=\varepsilon} \eta_{j} \Gamma_{0}(\eta) r^{-n}\left[\zeta_{x}(x)-\zeta_{x}{ }^{\prime} x\right)\right] d S_{y} B(x) \varphi(x) \\
& +\int_{r=\varepsilon} \eta_{j} \Gamma_{0}(\eta) r^{-n}\left[\zeta_{x}(x)-\zeta_{\alpha}(y)\right]\left[B(y) \varphi(y)-B(x) \varphi^{\prime}(x)\right] d S_{y} .
\end{aligned}
$$

The last term tends to zero with $\varepsilon$. Moreover, if the $\mathbf{T}_{\mathrm{A} \text { YLOR }}$ series for $\zeta_{x}(y)$ about $x$ is inserted in the first term on the right in (5.89) the result is

$$
\begin{aligned}
& -\int_{r=\varepsilon} \eta_{j} \Gamma_{0}(\eta) r^{-n} \nabla \zeta_{\alpha}(x) \cdot \operatorname{rnd} d S_{y} B(x) \varphi_{\rho}(x) \\
& \left.-\int_{r=\varepsilon} \eta_{j} \Gamma_{0}^{\prime} \eta_{j}\right) r^{-n+2}\left[\sum_{i, j=1}^{n} D_{i} D_{j} \zeta_{\alpha}\left(z_{x, y}\right)\right] \eta_{i} \eta_{j} d S_{y} B(x) f(x),
\end{aligned}
$$

where $z_{x, y}$ lies on the segment joining $x$ and $y$. The last term tends to zero with $\varepsilon$ and the first term equals the right-hand side of (5.87), which proves the lemma. 
Combining (5.71), (5.75) with Corollary 518 and Lemma 5.19 gives

Corollary 5.20 - The operator $\Pi_{\alpha j}$ has the representation

$$
\begin{aligned}
\Pi_{\alpha j \dot{\varphi}}(x) & =\int_{R^{n}}\left(D_{x_{j}} \Gamma_{0}(\eta) r^{-n}\right)\left[\zeta_{x}(x)-\zeta_{\alpha}(y)\right] B(y) \varphi(y) d y \\
& -\zeta_{x}(x) \int_{R^{n}} \Gamma_{0}(\eta) r^{-n}\left[D_{j} B(y)\right] \varphi(y) d y \\
& \left.+\int_{R^{n}} \Gamma_{0}(\eta) r^{-n} D_{j} \zeta_{x}(y) B(y)\right] \varphi(y) d y \\
& -\left(\sum_{k=1}^{n} D_{k} \zeta_{x}(x) \beta_{k j}\right) B(x) \varphi(x)
\end{aligned}
$$

for almost every $x \in R^{n}$ if $\varphi \in C_{0}^{\mathrm{I}}\left(R^{n} ; C^{m}\right)$.

Corollary 5.20 is now used to prove inequality (5.73). For this purpose a partition of unity $\left\{\zeta_{\alpha}\right\}$ is used which is the same as the partition $\left\{\zeta_{\alpha}\right\}$ of Theorem 3.5 but with cubes of twice the size $(d \rightarrow 2 d)$. Thus $\xi_{\alpha}=\xi_{\alpha}^{\prime}\left[\sum_{\beta} \xi_{\beta}^{\prime 2}\right]^{-1 / 2}$ where $\xi_{\beta}^{\prime}(x)=\xi^{\prime}(x-d \beta)$ and $\xi^{\prime}(x) \in C_{0}^{\infty}\left(R^{n}\right), 0 \leq \xi^{\prime}(x) \leq 1$, and supp $\xi^{\prime} \subset 2 Q_{0}$ where

$$
2 Q_{\alpha}=\left\{x: d\left(\alpha_{k}-2\right)<x_{k}<d\left(\alpha_{k}+2\right), k=1,2, \ldots, n\right\} .
$$

$\xi^{\prime}(x)$ is chosen so that

$$
\xi^{\prime}(x)= \begin{cases}1 & \text { for }-\frac{3}{2} d<x_{k}<\frac{3}{2} d, k=1, \ldots, n, \text { and } \\ 0 & \text { for } x \notin 2 Q_{0} .\end{cases}
$$

Clearly, supp $\xi_{x} \subset 2 Q_{\alpha}$. Moreover, apart from a null set every $x \in R^{n}$ lies in precisely $4^{n}$ of the cubes $2 Q_{\alpha}$. Also, note that $\zeta_{\alpha}(y)\left[1-\xi_{\alpha}^{\prime}(y)\right]=0$ for all $y$ and $\zeta_{\alpha}(x)\left[1-\zeta_{\alpha}^{\prime}(y)\right]=0$ unless $|x-y|>d / 2$. Finally, the derivatives $D^{r} \zeta_{\alpha}$. are bounded by a fixed constant for all $\alpha$.

Returning to Corollary 5.20, the function $\xi_{x}^{\prime}$ can be used to represent $\Pi$ ij $\varphi$ as follows:

$$
\begin{aligned}
\Pi_{\alpha j} \varphi(x) & =\int_{R^{n}}\left(D_{x_{j}} \Gamma_{0}(\eta) r^{-n}\right)\left[\zeta_{\alpha}(x)-\zeta_{\alpha}(y) \xi_{\alpha}^{\prime}(y) B(y) \varphi(y) d y\right. \\
& +\int_{R^{n}}\left(D_{x_{j}} \Gamma_{0}(\eta) r^{-n}\right)\left[\zeta_{\alpha}(x)-\zeta_{\alpha}(y)\right]\left(1-\xi_{\alpha}^{\prime}(y)\right) B(y) \varphi(y) d y
\end{aligned}
$$




$$
\begin{aligned}
& --\zeta_{\alpha}(x) \int_{R^{n}} \Gamma_{0}(\eta) r^{-n}\left[D_{j} B(y)\right] \varphi(y) d y \\
& +\int_{R^{n k}} \Gamma_{0}(\eta) r^{-n}\left[D_{j} \zeta_{\alpha}(y) B(y)\right] \varphi(y) d y-\left(\sum_{k=1}^{n} D_{k} \zeta_{\alpha}(x) \beta_{k j}\right) B(x) \varphi(x)
\end{aligned}
$$

Using the TAyLon series for $\zeta_{x}(y)$ about $x$ and $\zeta_{\alpha}(y)\left[1-\xi^{\prime}(y)\right]=0$ gives

$$
\begin{aligned}
& \Pi_{\alpha j} p(x)=-\sum_{k=1}^{n} D_{k} \zeta_{x}(x) \int_{R^{n}} r \eta_{k}\left(D_{x_{j}} \Gamma_{0}(\eta) r^{-n}\right) \xi_{\alpha}^{\prime}(y) B(y) \varphi(y) d y \\
& -2^{-1} \int_{R^{n}}\left(D_{x_{j}} \Gamma_{0}(\eta) r^{-n}\right) r^{2}\left[\sum_{k=1}^{n} D_{i} D_{k} \zeta_{\alpha}\left(z_{x, y}\right) \eta_{i} \eta_{k}\right] \xi_{x}^{\prime}(y) B(y) \varphi^{\prime}(y) d y \\
& +\zeta_{\alpha}(x) \int_{R^{n}}\left(D_{x_{j}} \Gamma_{0}(\eta) r^{-n}\right)\left(1-\zeta_{\alpha}^{t}(y)\right) B(y) \xi(y) d y \\
& -\zeta_{\alpha}(x) \int_{R^{n}} \Gamma_{0}(\eta) r^{-n}\left[D_{j} B(y)\right] \%(y) d y \\
& +\int_{R^{n}} \Gamma_{0}(\eta) r^{-n}\left[D_{j} \zeta_{\alpha}(y) B(y)\right] \Psi(y) d y \\
& -\left(\sum_{k=1}^{n} D_{k} \zeta_{x}(x) \beta_{k j}\right) B(x) \varphi(x) \\
& =\sum_{k=1}^{n} S_{j \alpha}^{k} \psi(x)+W_{j \alpha} \psi(x)+U_{j x} \psi(x)+V_{j \alpha}^{1} \varphi(x)+V_{j x \neq}^{2} \neq(x)+Z_{j \alpha} \varphi(x)
\end{aligned}
$$

where $\psi=B \varphi$ and the operators $S_{j x}^{k}, \ldots, Z_{j x}$ are defined by terms of the sum (5.95) in an obvious way. The left-hand side of inequality (5.73) can now be written

$$
\begin{gathered}
\left.\sum_{\alpha} \int_{R^{n}} \zeta_{\alpha} \Pi_{x j} \varphi\right|^{2} d x \\
=\sum_{\alpha} \int_{R^{n}} \mid \zeta_{x}(x)\left[\sum_{k=1}^{n} S_{j \alpha}^{k} \psi(x)+W_{j \alpha} \psi(x)+U_{j \alpha} \psi(x)+V_{j \alpha}^{1} \varphi(x)\right. \\
\left.+\nabla_{j \alpha}^{2} \varphi(x)+Z_{j x} \psi(x)\right]\left.\right|^{2} d x \\
\leq 6 \sum_{\alpha} \int_{R^{n}}\left\{\left|\zeta_{\alpha}(x) \sum_{k=1}^{n} S_{j \alpha}^{k} \psi(x)\right|^{2}+\left|\zeta_{\alpha}(x) W_{j \alpha} \psi(x)\right|^{2}\right.
\end{gathered}
$$




$$
\begin{aligned}
& +\left.\left|\zeta_{x}(x) U_{j \alpha} \psi(x)_{1}{ }^{2}+\right| \zeta_{\alpha \alpha}(x) \nabla_{j \alpha}^{1} \rho(x)\right|^{2}+\left|\zeta_{\alpha}(x) \nabla_{j \alpha}^{2} \rho^{\prime}(x)\right|^{2} \\
& \left.+\left|\zeta_{x}(x) Z_{j \times} \psi(x)\right|^{2}\right\} d x \\
& \leq 6 n \sum_{k=1}^{n} \sum_{x} \int_{R^{n}}\left|\zeta_{x}(x) S_{j 2}^{k} \psi(x)\right|^{2} \\
& +6 \sum_{\alpha} \int_{R^{n}}\left\{\left|\zeta_{x}(x) W_{j x} \psi^{\prime}(x)\right|^{2}+\left|\zeta_{x}(x) U_{j x} \psi(x)\right|^{2}+\left|\zeta_{\alpha}(x) V_{j x}^{1} \varphi(x)\right|^{2}\right. \\
& +\mid \zeta_{\alpha}\left(x,\left.V_{j \alpha}^{2} \varphi(x)\right|^{2}+\left|\zeta_{\alpha}(x) Z_{j \alpha} \psi^{\prime}(x)\right|^{2}\right\} d x \text {. }
\end{aligned}
$$

It will now be shown that each term on the right-hand side of this inequality if bounded by a constant times $\|\varphi\|^{2}$. This will complete the proof of inequality (5.73) and Theorem 5.15.

Lema 5.21. - There exists a constant $c_{2}>0$ such that

$$
\sum_{\alpha} \int_{R^{n}}\left|\zeta_{x}(x) S_{j \alpha}^{k} \psi(x)\right|^{2} d x \quad c_{2}\|\varphi\|^{2}
$$

for all $\psi \in C_{0}^{1}\left(R^{n} ; C^{m}\right)$. where

$$
\text { Proof. - Define } \mu_{\alpha}=\xi_{\alpha}^{\prime} \psi \text { so that (see }(5.95) \text { ) } S_{j \alpha}^{k} \psi(x)=-D_{k} \zeta_{\alpha}(x) S_{j}^{k} \mu_{\alpha}(x)
$$

$$
S_{j}^{k} \mu_{x}(x)=\int_{R^{n}} r \eta_{k}\left(D_{x_{j}} T_{0}(\eta) r^{-n}\right) \mu_{x}(y) d y .
$$

Then $\left|S_{j x}^{k} \psi(x)\right|^{2} \leq K\left|S_{j}^{k} \mu_{\alpha}(x)\right|^{2}$, where $K$ is a constant, because the derivatives of $\zeta_{x}$ are bounded, uniformly in $\alpha$. Moreover, $S_{j}^{k}$ is a bounded operator on $H_{0}$ (cf. $\left.[8,16]\right)$, and therefore

$$
\begin{aligned}
& \left.\sum_{\alpha} \int_{R^{n}}\left|\zeta_{\alpha}(x) S_{j x}^{k} \psi(x)\right|^{2} d x \leq K \quad \sum_{\alpha} \int_{R^{n}} \zeta_{\alpha}^{2} x\right)\left|S_{j}^{k} \mu_{\alpha}(x)\right|^{2} d x \\
\leq K & \sum_{\alpha} \int_{Q_{\alpha}}\left|S_{j}^{k} \mu_{x}(x)\right|^{2} d x \leq K \quad \sum_{\alpha}\left\|S_{j}^{k} \mu_{\alpha}\right\|^{2} \\
\leq K & \left.\sum_{\alpha}\left|S_{j}^{k}\left\|^{2}\right\| \mu_{\alpha}\left\|^{2}=K\right\| S_{j}^{k} \|^{2} \quad \sum_{\alpha} \int_{R^{n}}\left[\xi_{\alpha}^{\prime}(y)\right]^{2}\right| \psi(y)\right|^{2} d y
\end{aligned}
$$




$$
\leq K\left\|S_{j}^{k}\right\|^{2} \quad \sum_{\alpha} \int_{2^{2}}|\psi(x)|^{2} \leq 4^{n} K\left\|S_{j}^{k}\right\|^{2}\|\psi\|^{2}
$$

which proves the lemma.

LEMma 5.22. - There exists a constant $c_{3}>0$ such that

$$
\Sigma_{\alpha} \int_{R^{n}}\left|\zeta_{\alpha}(x) W_{j \propto} \Psi(x)\right|^{2} d x \leq c_{3}\|\Psi\|^{2}
$$

for all $\psi \in C_{0}^{l}\left(R^{n} ; C^{m}\right)$.

Proof. - The kernel of the integral defining $W_{j \pi}$ is $O\left(r^{1-n}\right)$ for $r=x-y_{i}^{\prime} \rightarrow 0$; see $(5,95)$. Hence

$$
\begin{aligned}
\left|\zeta_{x}(x) W_{j \alpha} \psi(x)\right| & \leq K_{1} \zeta_{x}(x) \int_{R^{n}}\left|\xi_{x}^{\prime}(y) \psi(y)\right| r^{1-n} d y \\
& \leq K_{1} \zeta_{x}(x) \int_{2 Q_{x}}|\psi(y)| r^{1-n} d y
\end{aligned}
$$

Hence

$\left|\zeta_{x}(x) W_{j \alpha} \psi(x)\right|^{2} \leq K_{1}^{2} \zeta_{x}^{2}(x) \int_{2 Q_{\alpha}} r^{1-n} d y \int_{2 Q_{\alpha}} r^{1-n}|\Psi(x)|^{2} d y$

$$
\left.\leq K_{1}^{2} 2 \sqrt{n d} \Omega_{n} \zeta_{x^{\prime}}^{2} x\right) \int_{2 Q_{\alpha}} r^{\cdot 1-n} d y \int_{2 Q_{\alpha}} r^{1-n}|\psi(y)|^{2} d y
$$

where $2 \sqrt{n d}$ is the radius of cireumscribed sphere abont $2 Q_{x}$. Thus

$$
\begin{aligned}
& \sum_{\alpha} \int_{R^{n}}\left|\zeta_{\alpha}(x) W_{j \alpha} \psi(x)\right|^{2} d x \leq\left. K_{2} \quad \sum_{z} \int_{R^{n}} \overbrace{a}^{2}(x) d x \int_{2 Q_{\alpha}} r^{1-n} \psi(y)\right|^{2} d y \\
& \leq\left. K_{2} \sum_{\alpha} \int_{Q_{\alpha}} d x \int_{2 Q_{\alpha}} r^{1-n}\left|\psi(x)^{2} d y=K_{2} \quad \sum_{\alpha}^{\Sigma} \int_{2 Q_{\alpha}}\right| \psi(x)\right|^{2} d y \int_{Q_{\alpha}} r^{1-n} d x \\
&(5.103) \quad \leq K_{2} \sqrt{n d} \Omega_{n} \sum_{x} \int_{2 Q_{\alpha}}|\psi(y)|^{2}=K_{2} \sqrt{n d} \Omega_{n}{ }^{4} n \mid \psi \|^{2}
\end{aligned}
$$

which proves $(5.100)$. 
LemMa 5.23. - There exists a constant $C_{4}>0$ such that

$$
\sum_{\alpha} \int_{R^{n}}\left|\zeta_{\alpha}(x) U_{j \alpha} \psi(x)\right|^{2} d x \leq O_{4}\|\Psi\|^{2}
$$

for all $\psi \in C_{0}^{1}\left(R^{n} ; C^{m}\right)$.

Proof. - The kernel $D_{x_{i}} \Gamma_{0}(\eta) r^{-n}=O\left(r_{-n-1}^{-n}\right)$ for $r \rightarrow 0$. Thus

$$
\left|U_{j \alpha} \psi(x)\right| \leq K_{3} \zeta_{\alpha}(x) \int_{R^{n}} r^{-n-1}\left[1-\xi_{\alpha}^{\prime}(y)\right]|\psi(y)| d y .
$$

Moreover, $\zeta_{\alpha}(x)\left[1-\xi_{x}(y)\right]=0$ unless $|x-y|>d / 2$. Hence for fixed $x$ the integral extends overs the set $\{y: r=|x-y|>d / 2\}$. Hence, by the SCHWARz inequality,

$$
\begin{aligned}
|U \psi(x)|^{2} & \leq K_{3}^{2} \zeta_{\alpha}^{2}(x) \int_{r>d / 2} r^{-n-1} d y \int_{r>}\left[1-\xi_{\alpha}^{\prime}(y)\right]^{2}|\Psi(y)|^{2} r^{-n-1} d y \\
& \leq K_{3}^{2} 2 d^{-1} \Omega_{n} \zeta_{\alpha}^{2}(x) \int_{r>d / 2}\left[1-\xi_{x}^{\prime}(y)\right]^{2}\left|\psi^{\prime}(y)\right|^{2} r^{-n-1} d y \\
& \leq K_{4} \int_{r<d / 2}\left[1-\xi_{\alpha}^{\prime}(y)\right]^{2}|\Psi(y)|^{2} r^{-n-1} d y
\end{aligned}
$$

Thus

$$
\begin{aligned}
& \sum_{\alpha}\left.\int_{R^{n}} \mid \zeta_{x}(x) U_{j, \alpha}\right\rfloor\left.(x)\right|^{2} d x \\
& \leq K_{4} \sum_{x} \int_{R^{n}} d x \int_{r>d / 2} \zeta_{x}^{2}\left(x\left[1-\xi_{\alpha}^{\prime}(y)\right]^{2}|\psi(y)|^{2} \gamma^{-n-1} d y\right.
\end{aligned}
$$

$$
\begin{aligned}
& \leq K_{*} \int_{R^{n}}|\Psi(y)|^{2} d y \int_{r>d / 2} \sum_{x} \zeta^{2}(x) r^{-n-1} d x \\
& =K_{4} 2 d^{-1} \Omega \int_{R^{n}} \mid \Psi(y)^{2} d y=K_{4} 2 d^{-1} \Omega_{n}\|\psi\|^{2},
\end{aligned}
$$

which proves (5.104). 
LemaA 5.24. - There exists a constant $c_{5}>0$ such that

$$
\sum_{\alpha} \int_{R^{n}}\left|\zeta_{\alpha}{ }^{\prime}(x) V_{j x}^{i} \varphi(x)\right|^{2} d x \leq c_{5}\|\varphi\|^{2}
$$

for all $\varphi \in C_{0}^{\mathrm{I}}\left(R^{n} ; C^{m}\right)$ and $i=1,2$.

Proof. - The proof will be given for $i=1$; the case $i=2$ is essentially the same. Let

$$
\left.V \mu(x)=\int_{R^{n}} \Gamma_{0}, \eta\right) r^{-n} \mu(y) d y .
$$

Then $V$ is bounded on $H_{0}$. Moreover, if $\mu_{j x}=\left(I_{j=\alpha}^{\circ} B\right) p$

$$
V_{j x \mathcal{f}}^{1}(x)=V \mu_{j a}(x),
$$

whence

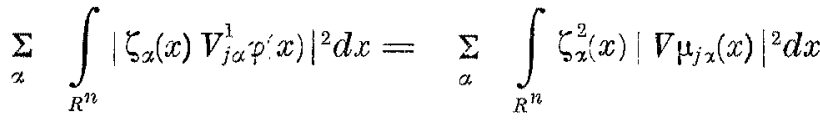

$$
\begin{aligned}
& \leq \sum_{\alpha} \int_{R^{n}} \mid \nabla \mu_{j x}(x)_{1}{ }^{2} d x \leq \Sigma_{\alpha}\left\|\nabla \mu_{j x}\right\|^{2}
\end{aligned}
$$

$$
\begin{aligned}
& \leq\|V\|^{2} \quad \sum_{\alpha}\left\|\mu_{j \alpha}\right\|^{2}=\|V\|^{2} \quad \sum_{\alpha} \int_{R^{2}}\left|\left(I_{j} \zeta_{\alpha}(y) B(y)\right) \xi(y)\right|^{2} d y \\
& \leq 2\|V\|^{2}\left\{\sum_{x} \int_{R^{n}}\left[D_{j} \zeta_{x}(y)\right]^{2}|B(y) \neq(y)|^{2} d y\right. \\
& \left.+\sum_{\star} \int_{R^{n}} \zeta_{\alpha}^{2}(y)\left|\left(D_{j} B(y)\right) \varphi(y)\right|^{2} d y\right\} \\
& \leq 2\|V\|^{2}\left\{K_{5} \quad \sum_{x} \int_{Q_{x}}|B(y) \varphi(y)|^{2} d y+\left\|\left(D_{j} B\right) \varphi\right\|^{2}\right\} \\
& \leq 2\|\nabla\|^{2}, 2^{n} K_{5}\|B \varphi\|^{2}+\left\|\left(D_{j} B\right) p\right\|^{2} \mid \leq C_{5}\|\varphi\|^{2} ;
\end{aligned}
$$

i e., (5.108) holds. 
Lemma 5.25. - There exists a constant $c_{6}>0$ such that

$$
\sum_{z} \int_{R^{n}}\left|\zeta_{\alpha}(x) Z_{j a} \psi(x)\right|^{2} d x \leq c_{6}\|\psi\|^{2}
$$

for all $\psi \in C_{0}^{\mathrm{1}}\left(R^{n} ; C^{m}\right)$.

Proof. - Clearly

$$
\left|Z_{j \times} \psi(x)\right|^{2} \leq K_{6}|\Psi(x)|^{2}
$$

whence

$$
\sum_{\alpha} \int_{R^{n}}\left|\zeta_{x}(x) Z_{j x} \psi(x)\right|^{2} d x \leq K_{6} \int_{R^{n}}|\psi(x)|^{2} d x=K_{6}\|\psi\|^{2}
$$

Lemma 5.21-5.25, with (5.96), sh)w that inequality (5.73) holds. This completes the proof of Theorem 5.15.

Returning now to the coerciveness inequality for $\Lambda^{\prime \prime}$, the proof of Theo* rem 5.14 will be based on the following two lemmas. The first depends on Theorem 515.

Lemma 5.26. - There exist positive constants $a_{1}$ and $\alpha_{2}$ such that for all $\varphi \in C_{0}^{1}\left(R^{n} ; C^{m}\right)$,

$$
\left\|\Lambda^{\prime} \varphi\right\|^{2} \geq 4^{-1} \sum_{a}\left\|\Lambda^{\prime} \zeta_{x} \varphi\right\|^{2}-a_{1}\|\varphi\|^{2}-a_{2}\|\varphi\| \cdot|\varphi|_{1}
$$

Proof - Definitions 5.4 and 5.5 and (5.40) imply

$$
\left\|\Lambda^{\prime} \varphi\right\|^{2}=\left\|\Lambda_{0}^{\prime} B_{f}\right\|^{2}=\left\|(-\Delta)^{1 / 2} P_{0} B \varphi\right\|^{2}=\sum_{j=1}^{n}\left\|D_{j} P_{0} B \varphi\right\|^{2} .
$$

Now $B \varphi=E_{0}^{-1} E_{\varphi} \rho$ has first derivatives in $H_{0}$, whence $D_{j} P_{0} B \varphi=P_{0} D_{j} B \varphi$. Thus (5.116) implies

$$
\begin{aligned}
\left\|\Lambda^{\prime} \varphi\right\|^{2} & =\sum_{j=1}^{n}\left\|P_{0} D_{j} B_{F}\right\|^{2}=\sum_{j=1}^{n}\left\|P_{0}\left(D_{j} B\right) \varphi+P_{0} B\left(D_{j} \varphi\right)\right\|^{2} \\
& \geq 2^{-1} \sum_{j=1}^{n}\left\|P_{0} B D_{j} \varphi\right\|^{2}-\sum_{j=1}^{n}\left\|P_{0}\left(D_{j} B\right) \varphi\right\|^{2} \\
& \geq 2^{-1} \sum_{j=1}^{n}\left\|P_{0} B D_{j} \varphi\right\|^{2}-c_{\lambda}\|\varphi\|^{2}
\end{aligned}
$$


where $c_{1}$ is a constant which depend on $E$ and $E_{0}$. Moreover, it is easy to verify that $\left\|P_{0} B D \varphi\right\|^{2}=\sum_{\alpha}\left\|\zeta_{\alpha} P_{0} B D_{j} \varphi\right\|^{2}$. Thus Theorem 5.15 implies

$$
\begin{gathered}
\left\|\Lambda^{\prime} \varphi\right\|^{2} \geq 2^{-1} \sum_{j 1}=\left\|P_{0} \zeta_{\alpha} B D_{j} \varphi\right\|^{2}-c_{2} \sum_{j=1}\|\varphi\| \cdot\left\|I_{j} \varphi\right\| \\
-c_{1}\|\varphi\|^{2}
\end{gathered}
$$

$$
\geq 2^{-1} \sum_{j=1}^{n} \Sigma\left\|P_{0} \zeta_{\alpha} B D \varphi\right\|^{2}-c_{3}\|\varphi\| \cdot|\varphi|_{1}-c_{1}\|\varphi\|^{2}
$$

Now

$$
\begin{aligned}
\left\|P_{0} B D_{j}\left(\zeta_{\alpha} \rho\right)\right\|^{2} & =\left\|P_{0} B\left(D_{j} \zeta_{\alpha}\right) \varphi+P_{0} B \zeta_{\alpha} D_{j} \varphi\right\|^{2} \\
& =\left\|P_{0} B\left(D_{j} \zeta_{\alpha}\right) \varphi\right\|^{2}+\left\|P_{0} \zeta_{\alpha} B D_{j} \varphi\right\|^{2} \\
& +2 \operatorname{Re}\left(B\left(D_{j} \zeta_{\alpha}\right) \varphi, ? P_{0} B \zeta_{\alpha} D_{j} \varphi\right) E_{0} .
\end{aligned}
$$

Hence, (5.118) can be rewritten

$$
\begin{aligned}
\left\|\Lambda^{\prime} \varphi\right\|^{2} & \geq 2^{-1} \sum_{j=1}^{n} \sum_{\alpha} i\left\|P_{0} B D_{j}\left(\zeta_{x} \varphi\right)\right\|^{2}-\left\|P_{0} B\left(D_{j} \zeta_{x}\right) \varphi\right\|^{2} \\
& -2 \operatorname{Re}\left(B\left(D_{\zeta_{\diamond}} \zeta_{x}\right) \varphi, P_{0} B_{\varsigma_{\alpha}}^{\llcorner} D_{j} \varphi\right)_{E_{0}} !-c_{3}\|\varphi\| \cdot|\varphi|_{1}-c_{1}\|\varphi\|^{2} .
\end{aligned}
$$

The second and third terms in braces are estimated next. First,

$$
\begin{aligned}
\left\|P_{0} B\left(D_{j \zeta_{\alpha}}\right) \varphi\right\|^{2} & \leq\left\|B\left(D_{j} \zeta_{\alpha}\right) \varphi\right\|^{2} \leq\|B\|_{\infty}^{2}\left\|\left(D_{j \zeta_{\alpha}}\right) \varphi\right\|^{2} \\
& \leq K_{1}\|B\|_{\infty}^{2} \int_{Q}|\varphi(x)|^{2} d x
\end{aligned}
$$

whence

$$
\begin{gathered}
2^{-1} \sum_{j=1}^{n} \sum_{\alpha}\left\|P_{0} B\left(D_{j} \zeta_{\alpha}\right) \varphi\right\|^{2} \\
\leq 2^{-1} n 2^{n} K_{1}\|B\|_{\infty}^{2}\|\varphi\|^{2}=c_{4}\|\varphi\|^{2} .
\end{gathered}
$$

Next,

$$
\begin{gathered}
\left|\left(B\left(D_{j} \zeta_{\alpha}\right) \varphi, P_{0} B \zeta_{\alpha} D_{j} \varphi\right)_{E_{0}}\right| \leq\left\|B\left(D_{j} \zeta_{\alpha}\right) \varphi\right\| \cdot\left\|P_{0} B_{\zeta_{\alpha}} D_{j} \varphi\right\| \\
\left.\left.\quad \leq K_{I}\|B\|_{\infty}^{2}\left\{\int_{Q_{\alpha}}|\varphi(x)|^{2} d x\right\}^{1 / 2} \int_{Q_{\alpha}} \mid D_{j} \varphi^{\prime} x\right)\left.\right|^{2} d x\right\}^{1 / 2}
\end{gathered}
$$


J. R. Schulenberger- C. H. Wrlcox: Coerciveness inequalities, etc. 289

whence

$$
\begin{aligned}
& \sum_{\alpha} \quad i\left(B\left(D_{j \subsetneq \alpha}^{\bullet}\right) \varphi, P_{0} B \zeta_{\alpha} D_{j} \varphi\right)_{E_{0}} \mid \\
& \leq K_{1}\|B\|_{\infty}^{2} \sum_{\alpha}\left\{\int_{Q_{\alpha}} \mid \varphi(x)^{2} d x\right\}^{1 / 2}\left\{\int_{Q_{x}}\left|D_{j} \varphi(x)\right|^{2} d x\right\}^{1 / 2} \\
& \leq K_{1}\|B\|_{\infty}^{2}\left\{\sum_{\alpha} \int_{Q_{\alpha}}|\varphi|^{2} d x\right\}^{1 / 2}\left\{\sum_{x} \int_{Q_{x}}\left|D_{j p}\right|^{2} d x\right\}^{1 / 2} \\
& =K_{1} 2^{n}\|B\|_{\infty O}^{2}\|\varphi\| \cdot\left\|D_{j} \varphi\right\|=c_{5}\|\varphi\| \cdot\left\|D_{J} \uparrow\right\| .
\end{aligned}
$$

Thus

$$
\sum_{j=1}^{n} \sum_{\alpha} \operatorname{Re}\left(B\left(D_{j \zeta}^{\zeta} x\right) \varphi, P_{0} B \zeta_{\zeta_{\alpha}} D_{j \zeta} \tau\right)_{E^{0}} \leq c_{5}\|\varphi\| \sum_{j=1}^{n}\left\|D_{j} \varphi\right\|
$$

$$
\leq c_{5} n\|\varphi\| \cdot \varphi_{1}=c_{6}\|\varphi\| \cdot|\varphi|_{1}
$$

Inequalities (5.120), (5.122) and (5.125) imply

$$
\begin{aligned}
&\left\|\Lambda^{\prime} \varphi\right\|^{2} \\
& \geq 2^{-1} \sum_{j=1}^{n} \sum_{x}\left\|P_{0} B D_{j}\left(\zeta_{x} \varphi\right)\right\|^{2}-c_{7}\|\varphi\|^{2}-c_{8}\|\psi\| \cdot|\varphi|_{1} .
\end{aligned}
$$

Now (5.116) implies that

$$
\begin{aligned}
\left\|\Lambda^{\prime} \zeta_{x} \varphi\right\|^{2} & =\sum_{j=1}^{n}\left\|P_{0} D_{j}\left(B \zeta_{x} \varphi\right)\right\|^{2} \\
& =\sum_{j=1}^{n}\left\|P_{0} B D_{j}\left(\zeta_{\alpha} \varphi\right)+P_{0}\left(D_{j} B\right)_{\varsigma_{\alpha}} \varphi\right\|^{2}
\end{aligned}
$$

whence

$$
\begin{aligned}
& \leq 2 \sum_{j=1}^{n}\left(\left\|P_{0} B D_{j}\left(\zeta_{\alpha} \varphi\right)\right\|^{2}+\left\|P_{0}\left(D_{j} B\right) \zeta_{\alpha} \varphi\right\|^{2}\right) \\
& \leq 2 \sum_{j=1}^{n}\left\|P_{0} B D_{j}\left(\zeta_{\alpha} \varphi\right)\right\|^{2}+2 K_{2} \sum_{j=1}^{n} \int_{Q_{\alpha}}|\varphi(x)|^{2} d x
\end{aligned}
$$

$$
\sum_{\alpha}\left\|\Lambda^{\prime} \zeta_{x} \varphi\right\|^{2} \leq 2 \sum_{j=1}^{n} \sum_{\alpha}\left\|P_{0} B D_{j}\left(\zeta_{x} \varphi\right)\right\|^{2}+2 K_{2} n 2^{n}\|\varphi\|^{2}
$$


Combining (5.126) and (5.128) gives

$$
\left\|\Lambda^{\prime} \varphi\right\|^{2} \geq 4^{-1} \quad \Sigma \quad\left\|\Lambda^{\prime} \zeta_{x} \varphi\right\|^{2}-a_{1}\|\varphi\|^{2}-a_{2}\|\varphi\| \cdot|\varphi|_{1} .
$$

LmMma 5.27. - There exist positive constants $a_{3}$ and $\alpha_{4}$ such that for all $\varphi \in C_{0}^{1}\left(R^{n} ; C^{m}\right)$,

$$
\|\Lambda \varphi\|^{2} \geq \sum_{\alpha}\left\|\Lambda \zeta_{a} \varphi\right\|^{2}-a_{3}\|\varphi\|^{2}-a_{4}\|\varphi\| \cdot|\varphi|_{1}
$$

Proor. - The partition of unity $\left\{\zeta_{\alpha}\right\}$ gives

$$
\begin{aligned}
\|\Lambda \varphi\|^{2} & =\sum_{\alpha}\left\|\zeta_{\alpha} \Lambda \varphi\right\|^{2} \\
& \left.=\sum_{\alpha}\left\|\Lambda \zeta_{\alpha} \varphi\right\|^{2}-i \sum_{\alpha}\left\|\left(\Lambda \zeta_{\alpha}\right) \varphi\right\|^{2}+2 \operatorname{Re}\left(\zeta_{\alpha} \Lambda \varphi,\left(\Lambda \zeta_{\alpha}\right) \varphi\right)_{E_{0}}\right\}
\end{aligned}
$$

The following estimates hold for the terms in braces

$$
\sum_{\alpha}\left\|\left(\Lambda \zeta_{\alpha}\right) \varphi\right\|^{2} \leq \sum_{\alpha} \int_{Q_{\alpha}}\left|\left(\Lambda \zeta_{\alpha}\right) \varphi\right|^{2} d y \leq K^{2} \sum_{\alpha} \int_{Q_{x}}|\varphi|^{2} \mathrm{~d} x=K_{2} 2^{n} \int_{R^{n}}|\varphi|^{2} d x .
$$

Also

$$
\begin{aligned}
& \left|\left(\zeta_{x} \rho,\left(\Lambda_{\zeta \alpha}^{\infty}\right)\right)_{E_{0}}\right| \\
& \leq c_{1} \int_{Q_{\alpha}}\left|\Lambda_{0} \varphi\right| \cdot|\varphi| d x \leq c_{1}\left\{\int_{Q_{\alpha}}\left|\Lambda_{0} \rho\right|^{2} d x\right\} 1 / 2\left\{\int_{Q_{\alpha}}|\varphi|^{2} d x\right\}^{1 / 2} .
\end{aligned}
$$

Moreover, $\left\|\Lambda_{0} \varphi\right\| \leq c_{2}|\varphi|_{1}$. Thus

$$
\begin{aligned}
& \sum_{\alpha} 2 \operatorname{Re}\left(\zeta_{\alpha} \Lambda \varphi,\left(\Lambda \zeta_{\alpha}\right) \varphi\right)_{E_{0}} \\
& \leq c_{1} \quad \sum_{\alpha}\left\{\int_{Q_{\alpha}}\left|\Lambda_{0} \varphi\right|^{2} d x\right\}^{1 / 2}\left\{\int_{Q_{\alpha}}|\varphi|^{2} d x\right\}^{1 / 2} \\
& \leq c_{1}\left\{\sum_{\alpha} \int_{Q_{\alpha}}|\Lambda \varphi|^{2} d x\right\}^{1 / 2}\left\{\sum_{\alpha} \int_{Q_{\alpha}}|\varphi|^{2} d x\right\}^{1 / 2} \leq 2^{n} c_{1}\left\|\Lambda_{0} \nsucc\right\| \cdot\|\varphi\|
\end{aligned}
$$

$(5.134) \leq 2^{n} c_{1} c_{2}\|\varphi\| \cdot|\varphi|_{1} \equiv a_{5}\|\varphi\| \cdot|\varphi|_{1}$.

(5.130) follows from $(5,131),(5.132)$ and (5.134). 
I. R. Schulenderger - C. H. Wilcox: Coerciveness inequalities, etc. 291

Lemma 5.26 and 5.27 imply

Corollary 5.28. - There exist positive constants $c_{1}$ and $c_{2}$ such that

$$
\left\|\Lambda^{\prime \prime} \varphi\right\|^{2} \geq 4^{-1} \quad \sum_{\alpha}\left\|\Lambda^{\prime \prime \prime} \zeta_{x} \varphi\right\|^{2}-c_{1}\|\varphi\|^{2}-c_{2}\|\varphi\| \cdot|\varphi|_{1}
$$

Proof of Theorem 5.14. - Let $\rho$ be defined as in Theorem 5.13 and construct the partition of unity $\left\{\zeta_{a}\right\}$ such that the width $2 d$ of the cubes sati. sfies $2 d \sqrt{ } n<\rho$. Then Theorem 5.13 implies

$$
\left|\zeta_{x} \varphi\right|_{1}^{2} \leq \mu_{0}^{2}\left(\left\|\Lambda^{\prime \prime} \zeta_{x} \varphi\right\|^{2}+\left\|\zeta_{\alpha} \varphi\right\|^{2}\right)
$$

for all $\varphi \in C_{0}^{1}\left(R^{n} ; C^{m}\right)$ and all $\alpha$. Honce

$$
\sum_{\alpha} \mid \zeta_{\alpha} \varphi_{1}^{2} \leq \mu_{0}^{2}\left(\sum_{\alpha}\left\|\Lambda^{\prime \prime} \zeta_{\alpha} \varphi\right\|^{2}+\|\varphi\|^{2}\right)
$$

Now

$$
\begin{aligned}
\left|\zeta_{\alpha \rho}\right|_{1}^{2} & =\sum_{j=1}^{n}\left\|D_{j} \zeta_{\alpha} \rho\right\|^{2} \\
& \geq 2^{-1} \sum_{j=1}^{n}\left\|\zeta_{\alpha}\left(D_{j} p\right)\right\|^{2}-\sum_{j=1}^{n}\left\|\left(D_{j} \zeta_{\alpha}\right) \varphi\right\|^{2}
\end{aligned}
$$

whence

$$
\sum_{\alpha}\left|\zeta_{\alpha} \varphi\right|_{1}^{2} \geq 2^{-1} \sum_{j=1}^{n}\left\|D_{j}\right\|^{2}-K_{1}\|\varphi\|^{2}=2^{-1}|\varphi|_{1}^{2}
$$

$$
-K_{1}\|\varphi\|^{2}
$$

where $K_{1}$ is independent of $\varphi$. Combining (5.135), (5.137) and (5.139) gives

$$
\begin{aligned}
\left\|\Lambda^{\prime \prime} \varphi\right\|^{2} & \geq 4^{-1} \sum_{\alpha}\left\|\Lambda^{\prime \prime} \zeta_{x} \varphi\right\|^{2}-c_{1}\|\varphi\|^{2}-c_{2}\|\varphi\| \cdot \mid \varphi_{11}^{\prime} \\
& \geq 4^{-1} \mu_{0}^{-2} \sum_{\alpha}\left|\zeta_{x} \varphi\right|_{1}^{2}-c_{3}\|\varphi\|^{2}-c_{2}\|\varphi\| \cdot|\varphi|_{1} \\
& \geq 8^{-1} \mu_{0}^{-2}|\varphi|_{1}^{2}-c_{4}\|\neq\|^{2}-\left.c_{2}\|\varphi\| \cdot\right|_{1} .
\end{aligned}
$$

Now $2\|\varphi\| \cdot|\varphi|_{1} \leq \varepsilon|\varphi|_{1}^{2}+\varepsilon^{-1}\|\varphi\|^{2}$ for any $\varepsilon>0$. Thus

$$
\left\|\Lambda^{\prime \prime} \varphi\right\|^{2} \geq\left(8^{-1} \mu_{0}^{-2}-2^{-1} c_{2} \Xi\right)|\varphi|_{1}^{2}-\left(c_{4}+2^{-1} c_{2} \varepsilon^{-1}\right)\|\varphi\|^{2} .
$$

Choosing $\varepsilon=8^{-1} c_{2}^{-1} \mu_{0}^{-2}$ gives an inequality equivalent to (5 66).

This completes the proof of Theorem 5.14. 
The next step in proving the coerciveness of $A^{\prime \prime}$ is to prove

Theorex 5.29. - Under the hypotheses of Theorem 5.8,

$$
|u|_{1}^{2} \leq \mu_{1}^{2}\left(\left\|\Lambda^{\prime \prime} u\right\|^{2}+\|u\|^{2}\right)
$$

for all $u \in D\left(\Lambda^{\prime \prime}\right) \cap \mathcal{O}^{1}\left(R^{n} ; C^{m}\right)$, where $\mu_{1}$ is the constant of Theorem 5.14.

The proof proceeds via several lemmas. The parpose of the next two lemmas is to show that inequality (5.142) holds for all $u \in L_{2, m}^{1} \cap C^{1}$. To this end let $\varphi(t) \in C^{\infty}(R), 0 \leq \varphi(t) \leq 1 \varphi(t) \equiv 0$ for $t>1$ and $\varphi(t) \equiv 1$ for $t<0$. Define $\varphi_{R}(x)=\varphi(|x|-R) \in C_{0}^{\infty}\left(R^{n}\right)$ for $(R>0)$.

Lemma 5.30. - If $u \in D\left(\Lambda^{\prime \prime}\right) \cap C^{1}\left(R^{n} ; C^{m}\right)$ then $\varphi_{R} u \in D\left(\Lambda^{\prime \prime}\right)$.

Proow. - Since $u \in C^{1}\left(R^{n} ; C^{m}\right)$ and $E(x)$ is continuously differentiable, $J^{*} \varphi_{R} u=E_{0}^{-1} E \varphi_{R} u \in C_{0}^{1}\left(R^{n} ; C^{m}\right) \subset L_{2, m}^{1}$. Hence $P_{0} J^{*} \rho_{R} u \in L_{2, m}^{1}$ and therefore $\varphi_{R} u \in D\left(\Lambda^{\prime}\right)$. Moreover it is easy to verify, using Lemma 2.5 , that $u \in D(\Lambda) \mathrm{im}$ plies $\varphi_{R} u \in D(\Lambda)$ and $\Lambda \varphi_{R} u=\varphi_{R} \Lambda u+\left(\Lambda \varphi_{R}\right) u$. Hence $\varphi_{R} u \in D\left(\Lambda^{\prime}\right) \cap D(\Lambda)=D\left(\Lambda^{\prime \prime}\right)$.

Lemma 5.31. - Under the hypotheses of Theorem 5.8, inequality (5.142) holds for all $u \in L_{2, m}^{1} \cap C^{1}\left(R^{n} ; C^{m}\right)$.

Prook. - If $\rho_{R}$ is defined as in Lemma 5.30 then $\varphi_{R} u \in C_{0}^{1}\left(R^{n} ; C^{m}\right)$ and hence, by Theorem 5.14

$$
\mid \varphi_{R} u \stackrel{2}{1} \leq \mu_{1}^{2}\left(\left\|\Lambda^{\prime \prime} \varphi_{R} u\right\|\left\|^{2}+\right\| \varphi_{R} u \|^{2}\right)
$$

Now it is easy to verify that $\varphi_{R} u \rightarrow u$ and $D_{J}\left(\varphi_{R} u\right) \rightarrow D_{j} u$ in $H$.

Also

$$
\begin{aligned}
& \left\|\Lambda^{\prime} \varphi_{R} u-\Lambda^{\prime} u\right\|^{2}=\sum_{j=1}^{n}\left\|D_{j} P_{0} J^{*}\left(\varphi_{R} u-u\right)\right\|^{2}= \\
& \left.=\sum_{j=1}^{n} \| P_{0} D_{j} J^{*}{ }_{\varphi_{R}} u-u\right)\left\|\leq \sum_{j=1}^{n}\right\| D_{j} J^{*}\left(\varphi_{R} u-u\right) \|^{2} \\
& \leq 2 \sum_{j=1}^{n}\left(\left\|D_{j} E_{0}^{-1} E\right\|_{\infty}^{2}\left\|\varphi_{R} u-u\right\|^{2}+\left\|E_{0}^{-1} E\right\|_{D}^{2}\left\|D_{j}\left(\varphi_{R} u-u\right)\right\|^{2}\right.
\end{aligned}
$$

which tends to zero for $R \rightarrow \infty$. Similarly, $\Lambda f_{R} u \rightarrow \Delta u$ in $H$. Hence, inequality (5.142) can be obtained by making $R \rightarrow \infty$ in (5.143).

In order to prove Theorem 5.29 an inequality is needed whose proof is of comparable difficulty with that of Theorem 5.15. The next lemma serves to motivate the inequality. 
J. R. Schulenberger - C. H. Wilcox: Coerciveness inequalities, etc.

Lema 5.32. - Let, $u \in D\left(\Lambda^{\prime \prime}\right) \cap C^{3}\left(R^{n} ; C^{m}\right)$ and suppose that there exists a positive constant $M=M(u)$ such that

$$
\left\|\Lambda^{\prime} \varphi_{R} u\right\| \leq M
$$

for all $R>0$. Then inequality $(5.142)$ holds for $u$ where $\mu_{1}$, the constant of Theorem 5.14, does not depend on $u$.

Proof. - It will be shown that (5.145) implies $u \in L_{2, m}^{1}$. The result then follows from Lemma 5.31. Now $\varphi_{R} u \in C_{0}^{1}\left(R^{n} ; C^{m}\right)$ and hence (5.143) holds. Moreover, $\varphi_{R} u$ and $\Lambda_{\varphi_{R}} u$ are bounded in $H$, uniformly for $R>0$. Hence $\left|\varphi_{R} u\right|_{1}$ is bounded for $R>0$. Now

$$
\begin{aligned}
\left\|\varphi_{R} D_{j} u\right\| & =\left\|D_{j}\left(\varphi_{R} u\right)-\left(D_{j} \varphi_{R}\right) u\right\| \\
& \leq\left\|D_{j}\left(\varphi_{R} u\right)^{\prime}\right\|+\left\|\left(D_{j} \tau_{R}\right) u\right\| \leq\left|\varphi_{R} u\right|_{1}+c\|u\|
\end{aligned}
$$
where $c$ is independent of $R$. Thus $\lim _{R \rightarrow \infty}\left\|\varphi_{R} D_{j} u\right\|=\left\|D_{j} u\right\|<\infty(j=1, \ldots, n)$
and therefore $u \in L_{2, m}^{1}$.

Theorem 5.29 is an immediate consequence of Lemma 5.32 and

Theorem 5.33. - Under the hypotheses of Theorem 5.8, if $u \in D\left(\Lambda^{\prime \prime}\right) \cap$ $\cap C^{1}\left(K^{n} ; C^{m}\right)$ then there exists a constant $M=M(u)$ such that

$$
\left\|D_{j} P_{00} J^{*} \varphi_{R} u\right\| \leq M
$$

for all $R>0$.

Proof of Theorem 5.29 based on Theorem $5.33-(5.40)$ and (5.44) imply that

$$
\left\|\Lambda \varphi_{Q} u\right\|^{2}=\left\|(\cdot \Delta)^{1 / 2} P_{0} J^{*} \varphi_{R} u\right\|^{2}=\sum_{j=1}^{n}\left\|D_{j} P_{0} J{ }^{*} \varphi_{R} u\right\|^{2} .
$$

Thus (5.147) implies (5.145) and hence (5.142).

Proof of Theorem 5.33. - Put $\psi=J^{*} u$ and note that $D_{j} P_{0} J^{*} \varphi_{R} u=$ $=D_{j} P_{0} \rho_{R} J^{*} u=D_{j} P_{0} p_{R} \psi$. This can be written, using the decomposition $P_{0}=$ $=x_{0}+\tilde{P}_{0}$ of $(5.18)$, as follows.

$$
\begin{aligned}
D_{j} P_{0} \varphi_{R} \psi & =D_{j} P_{0} \varphi_{R} \psi-D_{j} \hat{P}_{R} P_{0} \psi+D_{j} \varphi_{R} P_{0} \psi \\
& =D_{j} \check{P}_{0} \tilde{F}_{R} \psi-D_{j} \varphi_{R} \tilde{P}_{0} \psi+\left(D_{j} \varphi_{R}\right) P_{0} \psi+\varphi_{R} D_{j} P_{0} \psi
\end{aligned}
$$

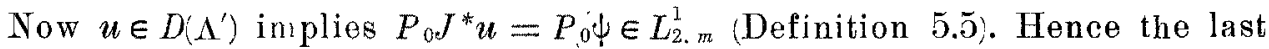
two terms in (5.149) are bounded in $H_{0}$ uniformly with respect to $R$. 
Thus, defining

$$
f_{R}=\tilde{P}_{0} \hat{p}_{R} \psi-\varphi_{R} \tilde{P}_{0} \psi
$$

it remains to show that $D_{j} f_{R}$ is bounded in $H_{0}$ uniformly with respect to $R$. 'This will be shown to follow from the next two lemmas.

Lqma 5.34. - Let $v \in C_{0}\left(R^{n} ; C^{m}\right)$ and define

$$
\begin{aligned}
\left.g_{R}{ }^{\prime} x\right) & \left.=\tilde{P}_{0 \rho_{R}} v^{\prime} x\right)-\varphi_{R}(x) \tilde{P}_{0} v(x) \\
& =\int_{R^{n}} \Gamma_{0}\left(\eta\left(r^{-n}\left[\varphi_{R}^{\prime} y\right)-\varphi_{R}^{\prime}(x)\right] v(y) d y\right.
\end{aligned}
$$

Then

$$
\begin{aligned}
D_{j} g_{R}(x) & =\int_{R^{n}}\left(D_{x_{j}} \Gamma_{0}(\eta) r^{-n}\right)\left[\varphi_{R}(y)-\varphi_{R}(x)\right](y) d y \\
& -\int_{R^{n}} \Gamma_{0}(\eta) r^{-n}\left[D_{j} \varphi_{R}(x)\right] v(y) d y-\left(\sum_{k=1}^{n} D_{k} \rho_{R}^{\prime}(x) \beta_{j k}\right) v(x)
\end{aligned}
$$

where

$$
\beta_{j k}=\int_{\Omega} \eta_{j} \eta_{k} \Gamma_{0}(\eta) \Omega_{\eta}
$$

Proof. - The integrand in (5.151) has a singularity of order $O\left(r^{-n+1}\right)$ for $R \rightarrow 0$. Hence (5.152) is just the formula for the differentiation of an integral with a weak singularity (cf. $[18, \mathrm{Ch}$. III], [16, p. 59]). The proof will not be repeated here. Note that the existence of the first integral on the right in (5.152) was proved in Corollary 5.18. (5.152) has nearly the same lorm as the representation of $I_{a j}$ in Corollary 5.20 .

Lemma 5.35. - Let $D_{j} g_{R}$ be given by (5.152) for $v \in C_{0}^{1}\left(R^{n} ; C^{m}\right)$, then there is a constant $N>0$, independent of $R$ and $v$, such that

$$
\left\|D_{i} g_{R}\right\| \leq N\|x\|
$$

for all $v \in C_{0}^{1}\left(R^{n} ; C^{m}\right)$.

Proof. - Note that $g_{R}=\tilde{P}_{0} \rho_{R} v-\varphi_{R} \tilde{P}_{0} v=P_{0} \rho_{R} v-\varphi_{R} P_{0} v \in L_{2, m}^{1}$ so that $D_{j} g_{R} \in H_{0}$. Therefore

$$
\left\|D_{j} g_{R}\right\|^{2}=\sum_{\alpha}\left\|C_{\alpha} D_{j} g_{R}\right\|^{2}
$$


J. R. Schulenberger - C. H. Wilcox: Coerciveness inequalities, etc. 295

Inequality (5.154) now follows from (5.152) in exactly the same way that (5.73) follows from (5.91) and Lemma 5.21-5.25. If these lemmas are applied to (5.152) the constant $N$ will be seen to depend on bounds for $\varphi_{R}$ and $D_{j} \varphi_{R}$. Since these are uniformly bounded in $R, N$ can be taken to be independent of $R$. This proves Lemma 5.35 .

Proof of Theorem 5.33 (completed). - To complete the proof it must be shown that $D_{j} f_{R}$ is bounded in $H_{0}$, uniformly in $R$, where $f_{R}$ is defined by (5.150). If $u \in C_{0}^{1}\left(R^{n} ; C^{m}\right)$ this follows from Lemma 5.35. For the general case, let $u \in D\left(\Lambda^{\prime \prime}\right) \cap C^{1}\left(R^{n} ; C^{m}\right)$ and let $\left\{u_{m} ! \subset C_{0}^{1}\left(R_{n} ; C_{m}\right)\right.$ converge to $u$ in $H_{0}$. Define $\psi=J^{*} u, \psi_{m}=J^{*} u_{m}$ and $f_{R}^{m}=\tilde{P}_{0 p_{R}} \psi_{m}-\varphi_{R} \tilde{P}_{0} \psi_{m}$. Then $f_{R}^{m} \rightarrow f_{R}$ when $m \rightarrow \infty$ because $\tilde{P}_{0}$ is a bounded operator on $H_{0}$. Moreover, Lemma 5.35 implies that

$$
\left\|D_{j} f_{R}^{m}\right\| \leq N\left\|\psi_{m}\right\|
$$

when $N$ is independent of $R .\left\{\psi_{m}\right.$ ? is a $\mathrm{C}_{A U C H Y}$ sequence in $H_{0}$ and therefore (5.156) implies that $\left\{D_{j} f_{R}^{m}\right\}$ is a $\mathrm{O}_{A \mathrm{UCH}} \mathrm{x}$ sequence in $H_{0}$. Hence $D_{j} f_{R}^{m} \rightarrow w$ in $H_{0}$ where $w$ is a weak derivative of $f_{R}$ in $H_{0}$. But (5.149) implies that $D_{j} f_{R} \in H_{0}$. Hence $w=D_{j} f_{R}$ by the uniqueness of weak derivatives. Thus passage to the limit $m \rightarrow \infty$ in (5.156) gives

$$
\left\|D_{j} f_{R}\right\| \leq N\|\psi\|=N\left\|J^{*} u\right\| \leq N^{\prime}\|u\|
$$

which completes the proof of Theorem 5.33.

Theorem 5.29 asserts that the coerciveness inequality holds for all $u \in D\left(\Lambda^{\prime \prime}\right) \cap C^{1}$. The next and final step is to extend it to all $u \in D\left(\Lambda^{\prime \prime}\right)$. This is the content of

Theorem 5.36. - Under the hypotheses of Theorem 5.8, $\Lambda^{\prime \prime}$ is $\Lambda$-coercive, and

$$
|u|_{1}^{2} \leq \mu_{i}^{2}\left(\left\|\Lambda^{\prime \prime} u\right\|^{2}+\|u\|^{2}\right)
$$

for all $u \in D\left(\Lambda^{\prime \prime}\right)=L_{2, m}^{1}$ where $\mu_{1}$ is the constant of Theorem 5.14.

The proof is given below after some preliminary lemmas. It will be convenient to define the operator

$$
\Gamma=\Lambda E^{-1}
$$

with domain $D(\mathbf{T})=\left\{u: E^{-1} u \in D(\Lambda) !\right.$. It follows from Theorem 2.6 and $(2.41)$ that $u \in D(T)$ and $\Gamma u=v$ if and only if

$$
\left(E^{-1} u, \Lambda^{+} \varphi\right)=(v, \varphi)
$$

for all $\varphi \in C_{0}^{1}\left(R^{n} ; C^{m}\right)$. Note that $\Lambda^{+}=A E^{-1}$, by $(2.40)$ and $(5.26)$. 
Moreover $E^{-1}$ is selfadjoint on $L_{2, m}$. Thus (5.160) implies

$$
\begin{gathered}
(v, \varphi)=\left(E^{-1} u, A E^{-1} \varphi\right)=\left(u, E^{-1} A E^{-1} \varphi\right) \\
=\left(u, \Delta E^{-1} \varphi\right)=(u, \Gamma \varphi)
\end{gathered}
$$

Moreover, it is easy to verify that the formal adjoint of $\Gamma$ on $L_{2, m}$ is $\Gamma^{+}=\Gamma$. Thus $u \in D(\Gamma)$ and $\Gamma u=v$ if and only if

$$
\left(u, \Gamma^{+} \varphi\right)=(v, \varphi)
$$

for all $\varphi \in C_{0}^{1}\left(R^{n} ; C^{m}\right)$. Hence, $\Gamma=\Gamma_{s}=\Gamma_{w}$, by Theorem 2.1.

Let $J_{k}$ denote Frienrichs' mollifier, "defined by (2.19). Then the following lemma is an immediate consequence of Corollary 2.2 and $\Gamma=\Gamma_{w}=\Gamma_{s}$.

Lemua 5.37. - If $u \in D(\Gamma)$ then $J_{k} u \in D(\Gamma)$ and $T J_{k} u \rightarrow \Gamma u$ in $H$ where $k \rightarrow \infty$.

Coroluart 5.38. - If $u \in D(\Lambda)$ then $E^{-1} J_{k} E u \in D(\Lambda)$ and $\Lambda E^{-1} J_{k} E u=T J_{k} E u$.

Proof. - It is clear from the definition of $D(\Gamma)$ that $u \in D(\Lambda)$ if and only if $E u \in D(\Gamma)$. Thus $u \in D(\Lambda)$ implies $J_{k} E u \in D(\Gamma)$, by Lemma 5.37, and this implies that $E^{-1} J_{k} E u \in D(\Lambda)$ and $\Lambda E^{-1} J_{k} E u=\Gamma J_{k} E u$. in $H$.

Lemma 5.39. - If $\left.u \in D, \Lambda^{\prime}\right)$ then $E^{-1} J_{k} E u \in D\left(\Lambda^{\prime}\right)$ and $\lim _{k \rightarrow \infty} \Lambda^{\prime} E^{-1} J_{k} E u=\Lambda^{\prime} u$

Proof. - By definition 5.5, $u \in D\left(\Lambda^{\prime}\right)$ if and only if $P_{0} J^{*} u \in L_{2, m}^{1}$. Now $E_{0}^{-1} J_{k}=J_{k} E_{0}^{-1}$, and it is easily verified that $P_{0} J_{k}=J_{k} P_{0}$, because $J_{k}$ is a scalar operator. Thus

$$
\begin{gathered}
P_{0} J^{*}\left(E^{-1} J_{k} E u\right)=P_{0} E_{0}^{-1} E\left(E^{-1} J_{k} E u\right) \\
=P_{0} E_{0}^{-1} J_{k} E u=P_{0} J_{k} E_{0}^{-1} E u=P_{0} J_{k} J^{*} u=J_{k}\left(P_{0} J^{*} u\right)
\end{gathered}
$$

Moreover; $v \in L_{2, m}^{1}$ implies $J_{k} v \in L_{2, m}^{1}$ by Corollary 2.2. Thus (5.163) implies

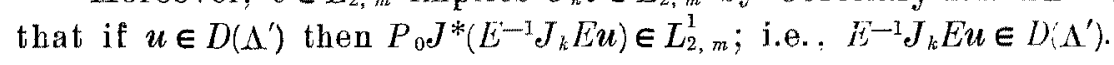

Next, note that

$$
\begin{aligned}
& \left\|\Lambda^{\prime}\left(E^{-1} J_{k} E u\right)-\Lambda^{\prime} u\right\|^{2}=\left\|\Lambda^{\prime}\left(E^{-1} J_{k} E u-u\right)\right\|^{2} \\
& =\sum_{j=1}^{n}\left\|D_{j} P_{0} J^{*}\left(E^{-1} J_{k} E u-u\right)\right\|^{2}=\sum_{j=1}^{n}\left\|D_{j} P_{0} E_{0}^{-1} E\left(E^{-1} J_{k} E u-u\right)\right\|^{2} \\
& =\sum_{j=1}^{n}\left\|D_{j} P_{0} J_{k} J^{*} u-D_{j} P_{0} J^{*} u\right\|^{2}=\sum_{j=1}^{n}\left\|J_{k}\left(D_{j} P_{0} J^{*} u\right)-D_{j} P_{0} J^{*} u\right\|^{2}
\end{aligned}
$$


J. R. Schulenberger - C. H. Wilcox: Coerciveness inequalities, etc. 297

The last term tends to zero when $k \rightarrow \infty$ because $D_{j} P_{0} J^{*} u \in H_{0}$, which proves the convergence statement.

Lemma 5.40. - If $u \in D\left(\Lambda^{\prime \prime}\right)$ then there exists a sequence $\left\{u_{k}\right\} \subset D\left(\Lambda^{\prime \prime}\right) \cap$ $\cap C^{1}\left(R^{n} ; C^{m}\right)$ such that $u_{k} \rightarrow u$ in $H$ and $\Lambda^{\prime \prime} u_{k} \rightarrow \Lambda^{\prime \prime} u$ in $L_{2,2 m}$.

Proof. - Define $u_{k}=E^{-1} J_{k} E u$. Then $u_{k} \rightarrow u$ in $H$, since $E^{-1}$ is a bounded operator. Moreover, $u_{k} \in C^{\mathrm{l}}\left(R^{n} ; C^{m}\right)$ because all functions in the range of $J_{k}$ are in $C^{\infty}\left(R^{n} ; C^{m}\right)$ and $E^{-1} \in C^{1}$. To complete the proof it suffices to prove that $u_{k} \in D\left(\Lambda^{\prime}\right) \cap D(\Lambda), \Lambda^{\prime} u_{k} \rightarrow \Lambda^{\prime} u$ in $H$ and $\Lambda u_{k} \rightarrow \Lambda u$ in $H$. But $u_{k} \in D\left(\Lambda^{\prime}\right)$ and $\Lambda^{\prime} u_{k} \rightarrow \Lambda^{\prime} u$ in $H$ by Lemma 5.39. Moreover, $u_{k} \in D(\Lambda)$ and $\Lambda u_{k}=\Gamma J_{k} E u$ by Corollary 5.38. Hence, by Lemma 5.37, $\Lambda u_{k}=\mathrm{T} J_{k} E u \rightarrow$ $\rightarrow \mathrm{I} E u=\Lambda u$ in $H$. This completes the proof.

Proon of Theorem 5.36. - Let $u \in D\left(\Lambda^{\prime \prime}\right)$ and let $\left\{\boldsymbol{u}_{k}\right\}$ be the sequence defined in Lemma 5.40. Then Theorem 5.29 is applicable to $u_{k}-u_{l}$ and gives

$$
\left|\boldsymbol{u}_{k}-\boldsymbol{u}_{l}\right|_{1}^{2} \leq \mu_{1}^{2}\left(\left\|\Lambda^{\prime \prime} \boldsymbol{u}_{k}-\Lambda^{\prime \prime} \boldsymbol{u}_{l}\right\|^{2}+\left\|\boldsymbol{u}_{k}-\boldsymbol{u}_{l}\right\|^{2}\right)
$$

Thus the convergence $u_{k} \rightarrow u$ in $H$ and $\Lambda^{\prime \prime} u_{k} \rightarrow \Lambda^{\prime \prime} u$ in $L_{2,2 m}^{1}$, guaranteed

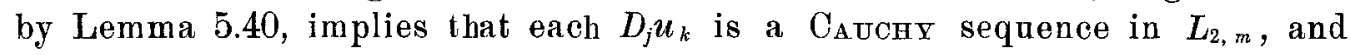
hence $D_{j} u_{k} \rightarrow v_{j} \in L_{2, m}$ for $j=1,2, \ldots, m$. Clearly, $v_{j}=D_{j} u$ in the sense of distribution theory. Thus $u \in L_{2, m}^{1}$ and passage to the limit $k \rightarrow \infty$ in the inequality

$$
\left|\boldsymbol{u}_{k}\right|_{1}^{2} \leq \mu_{1}^{2}\left(\left\|\Lambda^{\prime \prime} \boldsymbol{u}_{k}\right\|^{2}+\left\|\boldsymbol{u}_{k}\right\|^{2}\right)
$$

gives (5.158). This proves Theorem 5.36.

The goal of this section of the paper was to prove Theorem 5.8, the coerciveness of $\Lambda$ on $N(\Lambda)^{\perp}$. This is a corollary of Theorem 5.36.

Proof of Theorem 5.8. - Theorem 5.36 implies that if $u \in D\left(\Lambda^{\prime \prime}\right)=D(\Lambda) \cap$ $\cap D\left(\Lambda^{\prime}\right)=L_{2, m}^{1}$ then

$$
|u|_{1}^{2} \leq \mu_{1}^{2}\left(\|\Lambda u\|^{2}+\left\|\Lambda^{\prime} u\right\|^{2}+\|u\|^{2}\right)
$$

Moreover, Lemma 5.9 implies that $N(\Lambda)^{\perp} \subset N\left(\Lambda^{\prime}\right)$. Thus if $u \in D(\Lambda) \cap N(\Lambda)^{\perp}$ then $\left.\left.u \in D(\Lambda) \cap \Lambda^{\prime}\left(\Lambda^{\prime}\right) \subset D^{\prime} \Lambda\right) \cap D_{(}^{\prime} \Lambda^{\prime}\right)=D\left(\Lambda^{\prime \prime}\right)$ and $\Lambda^{\prime} u=0$. Hence (5.167) implies

$$
|u|_{1}^{2} \leq \mu_{1}^{2}\left(\|\Lambda u\|^{2}+\|u\|^{2}\right)
$$

for all $u \in D(\Lambda) \cap N(\Lambda)^{\perp}$. 


\section{§ 6. - Coerciveness Inequalities for Higher Order Derivatives.}

The purpose of this section is to generalize the cocrciveness theorem for $\Lambda$ (Theorem 5.8) to a coerciveness theorem for powers of $\Lambda$. It is shown below, under suitable assumptions on $E(x)$, that if $u \in D\left(\Lambda^{k}\right) \cap N(\Lambda, 1$ where $k$ is a positive integer then all the partial derivatives of $u$ of order $k$ are in $H$. This is done by generalizing the coerciveness theorem for $\Lambda^{\prime \prime}$ (Theorem 5.36) to one for powers of $\Lambda$ and $\Lambda^{\prime}$.

Let a general partial derivative operator be denoted by

$$
D^{x}=D_{1}^{\alpha_{1}} D_{2}^{\alpha_{2}} \ldots D_{n}^{x}
$$

(cf. (4.6)). The coerciveness theorems for h gher order operators can be formulated in terms of the Soboum spaces

$$
L_{2, m}^{k}=\left\{u: D^{\alpha} u \in L_{2, m} \text { for }|\alpha| \leq k\right\} \text {. }
$$

$L_{2, m}^{k}$ is a Hmbers space with inner product

$$
(u, v)_{k}=\int_{R^{\prime 3}} \sum_{x \mid \leq k}\left(D^{\alpha} u\right)^{*} D^{\alpha} v d \gamma
$$

and norm $\|u\|_{k}=(u, u)_{k}^{1 / 2}$. Clearly

$$
\|u\|_{k}^{2}=\sum_{|x| \leq k}\left\|D^{\alpha} u\right\|^{2}
$$

where $\|\cdot\|$ denotes the norm in $L_{2, m}$.

The principle result of this section is the following generalization of Theorem 5.36.

Theonem 6.1. - Let $k$ be a positive integer. If the hypotheses of Theorem 5.8 hold and, in addition, the derivatives $D^{x} E(x)$ are continuous and bounded on $R^{n}$ for $|\alpha| \leq k$ then

$$
D\left(\Lambda^{k}\right) \cap D\left(\Lambda^{\prime k}\right)=L_{2, m}^{k}
$$

and there exists a constant $\mu_{k}>0$ such that

$$
\|u\|_{k}^{2} \leq \mu_{k}^{2}\left(\left\|\Lambda^{k} u\right\|^{2}+\left\|\Lambda^{\prime k} u\right\|^{2}+\|u\|^{2}\right)
$$

for all $u \in D\left(\Lambda^{k}\right) \cap D\left(\Lambda^{\prime k}\right)$.

Note that $N(\Lambda)^{\perp} \subset N\left(\Lambda^{\prime}\right) \subset D\left(\Lambda^{\prime k}\right)$ for $k>1$. Hence, the following generalization of Theorem 5.8 is an immediate corollary of Theorem 6.1. 
J. R. Schulenberger - C. H. Wilcox: Coerciveness inequalities, etc.

Theorem 6.2. - Under the hypotheses of Theorem 6.1, $D\left(\Lambda^{k}\right) \cap N(\Lambda)^{\perp} \subset L_{2, m}^{k}$ and

$$
\|u\|_{k}^{2} \leq \mu_{k}^{2}\left(\left\|\Lambda^{k} u\right\|^{2}+\|u\|^{2}\right)
$$

for all $u \in D\left(\Lambda^{k}\right) \cap N(\Lambda)^{\perp}$.

Theorem 6.1 will be proved by induction on $k$. For $k=1$ the assertion of the theorem is true by Theorem 5.36. To complete the induction it will be shown that if the assertion of the theorem is true for an integer $k \geq 1$ then it is also true for $k+1$. Thus it will be shown that if the $D^{\times} E(x)$ are continuous and bounded for $|\alpha| \leq k+1$ and

$$
u \in D\left(\Lambda^{k+1}\right) \cap D\left(\Lambda^{k+1}\right)
$$

then $u \in L_{2, m}^{k+1}$ and there exists a constant $\mu_{k+1}$ such that

$$
\|u\|_{k+1}^{2} \leq \mu_{k+1}^{2}\left(\left\|\Lambda^{k+1} u\right\|^{2}+\left\|\Lambda^{\prime k+1} u\right\|^{2}+\|u\|^{2}\right) .
$$

In proving this, the inductive hypothesis is that $u \in L_{2, m}^{k}$ and (6.7) holds. The proof is given in a series of lemmas.

Lemma 6.3. - The hypothesis (6.8) implies that $\Lambda^{k} u$ and $\Lambda^{\prime k} u$ are in $L_{2, m}^{1}$.

Proor. - Let $v=\Lambda^{k} u$. Then $v \in D(\Lambda)$ by (6.8). Moreover, $\Lambda^{\prime} v=\Lambda^{\prime} \Lambda_{t}^{k}=$ $=\left(J\left(-\Delta^{1 / 2} P_{0} J^{*}\right)\left(J^{*-1} \Lambda_{0} J\right) \Lambda^{k-1} u=0\right.$ because $P_{0} \Lambda_{0}=0$. Thus $v \in N\left(\Lambda^{\prime}\right) \subset D\left(\Lambda^{\prime}\right)$. Hence $v \in D(\Lambda) \cap D\left(\Lambda^{\prime}\right)=D\left(\Lambda^{\prime \prime}\right)$ and therefore $v \in L_{2, m}^{1}$ by Theorem 5.36. Similarly, if $w=\Lambda^{\prime k} u$ then $w \in D\left(\Lambda^{\prime}\right)$ by (6.8). Morcover, $\Lambda w=\Lambda \Lambda^{\prime k}=E^{-1} J A J^{-1}$ $\left(J(-\Delta)^{1 / 2} P_{0} J^{*}\right) \Lambda^{1 / k-1} u=E^{-1} J(-\Delta)^{1 / 2} E_{0} \Lambda_{0} P_{0} J^{*} \Lambda^{1 k-1} u=0$ because $\Lambda_{0} P_{0}=0$. Thus $\left.w \in N(\Lambda) \subset D_{(}^{\prime} \Lambda\right)$. Hence $w \in D(\Lambda) \cap D\left(\Lambda^{\prime}\right)=D\left(\Lambda^{\prime \prime}\right)$ and therefore $w \in L_{2, m}^{1}$ by Theorem 5.36 .

The following notation is used to discuss difference quotients, $e_{j}=\mathrm{t}^{\mathrm{t}}(0$, $\ldots, 0,1,0, \ldots, 0)$ denotes the vector in $R^{n}$ with 1 as its $j^{\text {th }}$ component and 0 as the remaining components. The translation and difference operators for the $j^{\text {th }}$ coordinate are defined by

$$
\tau_{j, h} u(x)=u\left(x+h e_{j}\right)
$$

and

$$
\delta_{j, h} u(x)=h^{-1}\left[\tau_{j, h} u(x)-u(x)\right]
$$

where $h \neq 0$ is a real number.

Lемма 6.4 - If $u \in L_{2, m}^{k}$ then $\delta_{j, h} u \in D\left(\Lambda^{k}\right) \cap D\left(\Lambda^{\prime k}\right)$. 
This is evident since $\delta_{j, k} u \in L_{2, m}^{k}=D\left(\Lambda^{k}\right) \cap D\left(\Lambda^{\prime k}\right)$.

Lemma 6.5. - Let $k$ be a positive integer and define $\Lambda^{\circ}=I, \Lambda^{\prime 0}=I$. Then $\delta_{j, h} \Lambda^{\prime k} u$

$$
=\Lambda^{\prime k} \delta_{j, h} u+\sum_{l=1}^{k} \Lambda^{\prime k-l} J(-\Delta)^{1 / 2} P_{0}\left(\delta_{j, h} J^{*}\right) \tau_{j, h} \Lambda^{\prime l-1} u
$$

and

(6.13) $\delta^{j}{ }_{h} \Lambda^{k} u=\Lambda^{k} \delta_{j, h} u+\sum_{l=1}^{k} \Lambda^{k-l}\left(\delta_{j, h} J^{*-1}\right) \Lambda_{0} \tau_{j, h} \Lambda^{l-1} u$ for all $u \in L_{2, m}^{k}$.

Proof. - The proofs are by induction on $k$. Note that $\tau_{j, k}(-\Delta)^{1 / 2}=$ $=(-\Delta)^{1 / 2} \tau_{j, h}$ and $P_{0} \tau_{j, h}=\tau_{j, h} P_{0}$.

Proof of $(6.12)$. - To verify (6.12) for $k=1$ note that

$$
\left.\delta_{j, h} \Lambda^{\prime} u=\delta_{j, h} J(-\Delta)^{1 / 2} P_{0} J^{*} u=J(-\lrcorner\right)^{1 / 2} P_{0} \delta_{j, h}\left(J^{*} u\right)
$$

and

$$
\delta_{j, h}\left(J^{*} u\right)=J^{*} \delta_{j, h} u+\left(\delta_{j, h} J^{*}\right) \tau_{j, h} u
$$

Combining these gives

$$
\delta_{j, h} \Lambda^{\prime} u=\Lambda^{\prime} \delta_{j, h} u+J(-\Delta)^{1 / 2} P_{0}\left(\delta_{j, h} J^{*}\right) \tau_{j, h} u
$$

which is $(6.12)$ for $k=1$. Now if $(6.12)$ holds for $k$ then

$$
\delta_{j, h} \Lambda^{\prime k+1} u=\delta_{j, h} \Lambda^{\prime}\left(\Lambda^{\prime k} u\right)=\Lambda^{\prime} \delta_{j, h}\left(\Lambda^{\prime k} u\right)
$$

by the case $k=1$. Thus, by the inductive hypothesis,

$$
\begin{aligned}
\delta_{j, h} \Lambda^{\prime k+1} u & =\Lambda^{\prime k+1} \delta_{j, h} u+\sum_{=1}^{k} \Lambda^{\prime k+1-t} J(-\Delta)^{1 / 2} P_{0}\left(\delta_{j, h} J^{*}\right) \tau_{j, h} \Lambda^{\prime}- \\
& +J(-\Delta)^{1 / 2} P_{0}\left(\delta_{j, h} J^{*}\right) \tau_{j, k} \Lambda^{\prime k} u \\
& \left.=\Lambda^{\prime k+j} \delta_{j, h} u+\sum_{l=1}^{k+1} \Lambda^{\prime k+1-t} J(-J)^{1 / 2} P_{0} \delta_{j, h} J^{*}\right) \tau_{j, h} \Lambda^{\prime l-1}
\end{aligned}
$$

which completes the induction. 
Proof of (6.13). - Note that

$$
\begin{aligned}
\delta_{j, h} \Lambda u(x) & =\delta_{j, h} J^{*-1} \Lambda_{0} u(x) \\
& =h^{-1}\left[J^{*-1}\left(x+h e_{j}\right) \Lambda_{0} u\left(x+h e_{j}\right)+J^{*-1}(x) \Lambda_{0} u(x)\right] \\
& =J^{*-1}(x) \Lambda_{0} h^{-1}\left[u\left(x+h e_{j}\right)-u(x)\right] \\
& +h^{-1}\left[J^{*-1}\left(x+h e_{j}\right)-J^{*-1}(x)\right] \Lambda_{0} u\left(x+h e_{j}\right) \\
& =\Lambda \delta_{j, h} u(x)+\left(\delta_{j, h} J^{*-1}\right) \Lambda_{0} \tau_{j, h} u(x)
\end{aligned}
$$

which is $(613)$ for $k=1$. Now if $(6.13)$ holds for $k$ then

$$
\delta_{j, k} \Lambda_{--1}^{k+1} u=\delta_{j, k}\left(\Lambda_{-}^{k} u\right)
$$

$$
=\Lambda^{\delta}{ }_{j, h}\left(\Lambda^{k} u\right)+\left(\delta_{j, h} J^{*-1}\right) \Lambda_{0} \tau_{j, h} \Lambda^{k} u
$$

by the case $k=1$. Thus, by the inductive hypothesis,

$$
\begin{aligned}
\delta_{j, h} \Lambda^{k+1} u & =\Lambda^{k+1} \delta_{j, h} u+\sum_{l=1}^{k} \Lambda^{k+1-l}\left(\delta_{j, h} J^{*-1}\right) \Lambda_{0} \tau_{j, h} \Lambda^{l-1} u \\
& +\left(\delta_{j, h} J^{*-1}\right) \Lambda_{0} \tau_{j, h} \Lambda^{k} u \\
& =\Lambda^{k+1} \delta_{j, h} u+\sum_{l=1}^{k+1} \Lambda^{k+1-l}\left(\delta_{j, h} J^{*-1}\right) \Lambda_{0} \tau_{j, h} \Lambda^{l-1} u
\end{aligned}
$$

which completes the induction.

Lamas 6.6. - Let $0<h \leq h_{0}$. Then there exist constants $c_{k}^{\prime}$ and $c_{k}$, which depend only on $k, h_{0}, E_{0}, A_{j}$ and the constants $\left\|D^{*} E\right\|_{\infty}$ for $|\alpha| \leq k+1$, such that

$$
\sum_{l=1}^{k}\left\|\Lambda^{\prime k-l} J(-\Delta)^{1 / 2} P_{0}\left(\delta_{j, h} J^{*}\right) \tau_{j, h} \Lambda^{\prime l-1} u\right\|^{2} \leq c_{k}^{\prime}\|u\|_{k}^{2}
$$

and

$$
\sum_{i=1}^{k}\left\|\Lambda^{k-l}\left(\delta_{j, k} J^{*-1}\right) \Lambda_{0} \tau_{j, h} \Lambda^{l-1} u\right\|^{2} \leq c_{k}\|u\|_{k}^{2}
$$

for all $u \in L_{2, m}^{k}$.

Proor. - It is clear that the left-hand side of (6.22), for example, involves the norm of a linear combination of derivatives of $u$ of orders $k$ or less, with coefficients which involve derivatives $D^{x} \delta_{j, h} J^{*}=E_{0}^{-3} D^{x} \delta_{j, h} E$ of order 
$k$ or less. The latter can be majorized by terms of the form $\left\|D^{\times} E\right\|$ with $|\alpha| \leq k+1$. The proofs of $(6.22)$ and $(6.23)$ follows by a straightforward in. duction on $k$ which will not be given here.

The following standard lemma may ${ }_{2}^{*}$ be found in $[2$, p. 54].

LEMMA 6.7. - Let $u \in L_{2, m}^{k}$ and suppose that there exists positive constants $C$ and $h_{0}$ such that

$$
\left\|\delta_{j, h} u\right\|_{k} \leq C
$$

for $0<h \leq h_{0}$ and $j=1,2, \ldots, n$. Then $u \in L_{2, m}^{k+1}$ and

$$
\left\|D_{j} u\right\|_{k} \leq C
$$

for $j=1,2, \ldots, n$.

Lемма 6.8. - Let $\left\|D^{x} E\right\|_{\infty}<\infty$ for $|\alpha| \leq k+1$ and assume that (6.8) holds. Then $u \in L_{2, m}^{k+1}$ and there exists a constant $\mu_{h}^{\prime}$, depending only on $E_{0}$, $A_{j}$ and the $\left\|D^{x} E\right\|_{\infty}$ for $|\alpha| \leq k+1$, such that

$$
\|u\|_{k+1}^{2} \leq \mu_{k}^{\prime 2}\left(\left\|\Lambda^{k} u\right\|_{1}^{2}+\left\|\Lambda^{\prime k} u\right\|_{1}^{2}+\|u\|^{2}\right)
$$

for all $u$ satisfying (6.8).

Proof. - By the inductive hypothesis and Lemma 6.4, $\delta_{j, k} u \in D\left(\Lambda^{k}\right) \cap D\left(\Lambda^{\prime k}\right)$. Hence, the inductive hypothesis implies that $(6.6)$ holds for $\delta_{j, h} u$, which implies

$$
\left\|\delta_{j, k} u\right\|_{k}^{2} \leq \mu_{k}^{2}\left(\left\|\Lambda^{k \delta_{j, h} u}\right\|^{2}+\left\|\Lambda^{\prime k \delta_{j, h}} u\right\|^{2}+\left\|\delta_{j, h} u\right\|^{2}\right)
$$

Combining this with the results of Lemma 6.5 and 6.6 gives

$$
\begin{aligned}
& \left\|\delta_{j, h} u\right\|_{k}^{2} \\
& \quad \leq 2 \mu_{k}^{2}\left(\left\|\delta_{j, h} \Lambda^{k} u\right\|^{2}+\left\|\delta_{j, h} \Lambda^{\prime k} u\right\|^{2}+\left(c_{k}+c_{k}^{\prime}\right)\|u\|_{k}^{2}+\left\|\delta_{j, h} u\right\|^{2}\right) .
\end{aligned}
$$

Now Lemma 6.3 implies that the derivatives $D_{j} \Lambda^{k} u$ and $D_{j} \Lambda^{\prime k} u$ exist in $H$, and it follows that $\delta_{j, h} \Lambda^{k} u \rightarrow D_{j} \Lambda^{k} u$ and $\delta_{j, h} \Lambda^{\prime k} u \rightarrow D_{j} \Lambda^{\prime k} u$ in $H$ when $h \rightarrow 0$. Thus, the right-hand side of $(6.28)$ is bounded for $0<h \leq h_{0}$. It follows by Lemma $6.7 u \in L_{2, m}^{k+1}$. Moreover, passage to the limit $h \rightarrow 0$ in (6.28) gives

$$
\begin{aligned}
& \left\|D_{j} u\right\|_{k}^{2} \\
& \left.\quad \leq 2 \mu_{k_{i}}^{2}\left\|D_{j} \Lambda^{k} u\right\|^{2}+\left\|D_{j} \Lambda^{\prime k} u\right\|^{2}+\left(c_{k}+c_{k}^{\prime}\right)\|u\|^{2}+\left\|D_{j} u\right\|^{2}\right) .
\end{aligned}
$$


J. R. Schulenberger - C. H. Wilcox: Coerciveness inequalities, etc.

Summing over $j=1,2, \ldots, n$, adding $\|u\|^{2}$ to both sides, and noting that $\left|\Lambda^{k} u\right|_{1}^{2} \leq\left\|\Lambda^{k} u\right\|_{1}^{2}$ and $\left|\Lambda^{\prime k} u\right|_{1}^{2} \leq\left\|\Lambda^{\prime k} u\right\|_{1}^{2}$ gives

$$
\|u\|_{k+1}^{2}
$$

$$
\leq 2 \mu_{k}^{2}\left(\left\|\Lambda^{k} u\right\|_{1}^{2}+\left\|\Lambda^{\prime k} u\right\|_{1}^{2}+n\left(c_{k}+c_{k}^{\prime}\right)\|u\|^{2}+\|u\|_{1}^{2}\right) .
$$

Inequality (6.26) follows immediately from (6.30) and the following lemma.

Lemma 6.9 - Let $k \geq 1$ be an integer and let $j$ be an integer such that $0 \leq j \leq k$. Then given $\varepsilon>0$, there exists a constant $C=C(\varepsilon)$ such that for all $u \in L_{2, m}^{k+1}$

$$
\|u\|_{j}^{2} \leq \varepsilon\|u\|_{k+1}^{2}+C\|u\|^{2}
$$

Proof. - It can be shown by an elementary argument that for each $\varepsilon>0$ there exists a $C=C(\varepsilon)$ such that

$$
\underset{|\alpha| \leq j}{\Sigma} p^{2 \alpha} \leq \varepsilon \sum_{\mid \alpha^{i} \leq k+1} p^{2 \alpha}+C
$$

for all $p \in R^{n}$. (6.31) follows immediately from (6.32) by the Plancherel theory.

Lemma 6.10. - Let $\varepsilon>0$. Then (6.8) implies that there exists a constant $C_{1}$, depending only on $E_{0}, A_{j}$ and $\| D^{\alpha} E_{\infty}$ for $|\alpha| \leq k-1$, and a constant $C_{1}(\varepsilon)$ such that

$$
\left\|\Lambda^{k} u\right\|_{1}^{2}
$$

$$
\leq \mu_{1}^{2}\left[\left\|\Lambda^{k+1} u\right\|^{2}+\varepsilon C_{1}\|u\|_{k+1}^{2}+C_{2}(\varepsilon)\|u\|^{2}\right] .
$$

Proof. - Lemma 6.3 implies that $\Lambda^{k} u \in L_{2, m}^{1}$. Hence by Theorem 5.36 applied to $\Lambda^{k} u$,

$$
\left\|\Lambda^{k} u\right\|_{1}^{2} \leq \mu_{1}^{2}\left[\left\|\Lambda^{k+1} u\right\|^{2}+\left\|\Lambda^{\prime} \Lambda^{k} u\right\|^{2}+\left\|\Lambda^{k} u\right\|^{2}\right]
$$

$$
=\mu_{1}^{2}\left[\left\|\Lambda^{k+1} u\right\|^{2}+\left\|\Lambda^{k} u\right\|^{2}\right]
$$

since $\Lambda^{\prime} \Lambda^{k}=0$. Now $\left\|\Lambda^{k} u\right\|^{2} \leq C_{1}\|u\|_{k}^{2}$ where $C_{1}$ depends on $E_{0}, A_{j}$ an 1 $\left\|D^{\star} E\right\|_{\infty}$ for $|\alpha| \leq k-1$ only. Combining this with (6.31) and (6.34) gives (6.33).

Lemma 6.11. - Let $\varepsilon>0$. Then $(6.8)$ implies that there exists a constant $C_{1}$, depending ony on $E_{0}, A_{j}$ and $\left\|D^{\alpha} E\right\|_{\infty}$ for $|\alpha| \leq k$, and a constant $C_{4}(\varepsilon)$ such that

$$
\left\|\Lambda^{\prime k} u\right\|_{1}^{2} \leq \mu_{1}^{2}\left[\left\|\Lambda^{\prime k+1} u\right\|^{2}+\varepsilon C_{3}\|u\|_{k+1}^{2}+C_{4}(\varepsilon)\|u\|^{2}\right]
$$


Proof. - Lemma 6.3 implies that $\Lambda^{\prime k} u \in L_{2, m}^{1}$. Hence by Theorem 5.36 applied to $\Lambda^{\prime k} u$,

$$
\begin{aligned}
\left\|\Lambda^{\prime k} u\right\|_{1}^{2} & \leq \mu^{2}\left[\left\|\Lambda \Lambda^{\prime k} u\right\|^{2}+\left\|\Lambda^{\prime k+1} u\right\|^{2}+\left\|\Lambda^{\prime k} u\right\|^{2}\right] \\
& =\mu_{1}^{2}\left[\left\|\Lambda^{\prime k+1} u\right\|^{2}+\left\|\Lambda^{\prime k} u\right\|^{2}\right]
\end{aligned}
$$

since $\Lambda \Lambda^{\prime k}=0$. Now, it is easy to verify by induction that $\left\|\Lambda^{\prime k} u\right\|^{2} \leq C_{3}\|u\|_{k}^{2}$ for all $u \in L_{2, m}^{h}$ where $C_{3}$ depends on $E_{0} A_{j}$ and $\left\|D^{\alpha} E\right\|_{\infty}$ for $|\alpha| \leq k$ only. Combining with (6.31), (6.36) gives (6.35).

Proof of Theorem 6.1. - (6.8) and the inductive hypothesis imply that Lemmas $6.8,6.10$ and 6.11 hold. Combining these results gives

$$
\begin{aligned}
& \|u\|_{k+1} \leq \mu_{k}^{\prime 2}\left\{\mu _ { i } ^ { 2 } \left[\left\|\Lambda^{k+1} u\right\|^{2}+\left\|\Lambda^{\prime k+1}\right\|^{2}+\varepsilon\left(C_{1}+C_{3}\right)\|u\|_{k+1}^{2}\right.\right. \\
& \left.+\left(C_{2}(\varepsilon)+C_{4}^{\prime} \varepsilon\right)\|u\|^{2}\right]+\|u\|^{2} !
\end{aligned}
$$

Hence

$$
\left(1-\mu_{k}^{2} \mu_{1}^{2}\left(C_{1}+C_{3}\right) \varepsilon\right)\|u\|_{k+1}^{2}
$$

$$
\begin{aligned}
& \leq \mu_{k}^{\prime 2} \mu_{1}^{2}\left[\left\|\Lambda^{k+1} u\right\|^{2}+\left\|\Lambda^{\prime k+1} u\right\|^{2}\right] \\
& +\left(\mu_{k}^{\prime 2}+\mu_{k}^{\prime 2}\left(C_{2}(\varepsilon)+C_{4}(\varepsilon)\right)\|u\|^{2} .\right.
\end{aligned}
$$

Choosing $\varepsilon$ so that $1-\mu_{k}^{\prime 2} \mu_{1}^{2}\left(C_{1}+C_{3}\right) \varepsilon=\frac{1}{2}$ implies that (6.9) holds with a suitable constant $\mu_{k+1}$. This completes the induction and the proof of Theorem 6.1.

\section{$\S 7$. - Coneluding Remarks.}

The coerciveness theorems proved in this paper suggest a number of new problems in the theory of partial differential equations. One obvious problem is to generalize the coerciveness theorem to general first-order operators $L$ which are nonelliptic and have constant deficit. It is also of interest to extend the results to operators of higher order. In this connection it would be desirable to use the Douglis-NIrenserg notion of ellipticity [9] so that the equations of the system can have different orders. Another problem which is important for applications to problems in the theory of wave propagation and scattering is to extend the coerciveness theorems to boundary value problems for nonelliptic operators in bounded and unbounded domains. In this case, if the method of augmented operators is used it will be necessary to find suitable boundary conditions for the angmented operators. 
J. R. Schulenberger - C. H. Wilcox: Coerciveness inequalities, etc. 305

\section{REFERENCES}

[1] S. AGMon, The coerciveness problem for integro-differential forms, J. Analyse Math., 6, pp. 183.223 (1958).

[2] - - The $L_{p}$ approach to the Dirichlet problem, Ann. Scnola Norm Sup. Pisa, 13, pp. $405-448$ (1059).

[3] - - Lectures on elliptic boundary value problems, New York: Van Nostrand 1965.

[4] S. AGXON, A. DovgLIs and L. NIRENBerG, Estimates neat the boundary for solutions of elliptic partial differential equations satisfying general boundary conditions $I I$, Comm. Pure Appl. Math., 17, pp. 3592 (1964).

[5] N. Aronszajn, On coercive integro-differential quadratic forms, Conference on Partial Differential Equations, Univ. of Kansas, Te.h. Rep. No. 14, pp. $94-106$ (1954).

[6] G.S.S. AVILA and C.H. WILCox, The near-field behavior of the Green's matrix in anisotropic wave motion, J. Math. Mech., 16, pp. 867.583 (1967).

[7] A.P. Calderon and A. Zygmund, On singular integrals, Amer. J. Math., 78, pp. 289.309 (1956).

[8] - - Singular integral operators and differential equations, Amer. J. Math., 70, pp. 901.921 (1957).

[9] A. Dovglis and L. Nrrenberc, Interior estimates for elliptic systems of partial differential equations, Comm. Pure Appl. Math., 8, pp 503.538 (19อ̃5).

[10] G. Fromera, Lineav elliptic differential systems and eigenvalue problems, New York: Springer-Verlag 1965.

[11] D.G. de FrGUEIREDo. The coerciveness problem for forms over vector valued functions, Comm. Pure Appl. Math., 16, pp. 63-94 (1963).

[12] K. O. FRIEDRICHS. The identity of weak and strong extensions of differential operators, Trans. A. M S., 55, pp. 132-151 (1944).

[13] L. Hörmander, Linear partial differential operators, New York: Springer-Verlag 1963.

[14] T. Kato, Pertubation theory for linear operators, New Yolk: Springer-Verlag 1966.

[15] A. E.H. Love, The mathematical theory of elasticity, 4th edition, New York: Dover Publications 1944.

[16] S. G. Mikftin, Multidimensional singular integrals and integral equations, London: Pergamon Press 1965.

[17] M. Sohechter, Coerciveness of linear partial differential operator satisfying zero Di. richlet data, Comm. Pure Appl. Math., 11, pp. 153-174 (1958).

[18] J.R. SChULENBERGER, Wave propagation in inhomogeneous anisotropic media, Thesis, University of Arizona 1968.

[19] - -, The singular integral associated with a class of uniformly propagative systems. ONR Technical Summary Rept. No. 1 University" of Arizona, (August 1969).

[이 J.R. EChULENBERGER and C.H. WI.cox, Completeness of the wave operators for perturbations of uniformly propasative systems, J. Functional Anal (to appear, 1971).

[21] K. T. SMiтн, Inequalities for formally positive integro-differential forms, Bull. A.M.S., 67, pp. 368-370 (1961).

[22] C. H. WILCox, Wave operators and asymplotic solutions of wave propagation problems of classical physics, Arch. Rational Mech. Anal., 22, pp. 37.78 (1966).

[28] - - Steady-state wave propagation in homogeneous anisotropic media, Arch. Rational Meeh. Anal, 25, pp 201.242 (1967).

[24] - - Transient wave propagation in homogeneous anisotropic media, Arch. Rational Mech. Anal, 37, pp. 323.343 (1470). 